\title{
Verifying Custom Synchronisation Constructs Using Higher-Order Separation Logic
}

MIKE DODDS, University of York, UK

SURESH JAGANNATHAN, Purdue University, Indiana

MATTHEW J. PARKINSON, Microsoft Research, UK

KASPER SVENDSEN, Aarhus University, Denmark

LARS BIRKEDAL, Aarhus University, Denmark

\begin{abstract}
Synchronisation constructs lie at the heart of any reliable concurrent program. Many such constructs are standard - e.g., locks, queues, stacks, and hash-tables. However, many concurrent applications require custom synchronisation constructs with special-purpose behaviour. These constructs present a significant challenge for verification. Like standard constructs, they rely on subtle racy behaviour, but unlike standard constructs, they may not have well-understood abstract interfaces. As they are custom-built, such constructs are also far more likely to be unreliable.

This paper examines the formal specification and verification of custom synchronisation constructs. Our target is a library of channels used in automated parallelization to enforce sequential behaviour between program statements. Our high-level specification captures the conditions necessary for correct execution; these conditions reflect program dependencies necessary to ensure sequential behaviour. We connect the high-level specification with the low-level library implementation, to prove that a client's requirements are satisfied. Significantly, we can reason about program and library correctness without breaking abstraction boundaries.

To achieve this, we use a program logic called iCAP (impredicative Concurrent Abstract Predicates) based on separation logic. iCAP supports both high-level abstraction and low-level reasoning about races. We use this to show that our high-level channel specification abstracts three different, increasingly complex lowlevel implementations of the library. iCAP's support for higher-order reasoning lets us prove that sequential dependencies are respected, while iCAP's next-generation semantic model lets us avoid ugly problems with cyclic dependencies.
\end{abstract}

Categories and Subject Descriptors: D.2.4 [Software Engineering]: Program Verification-Correctness proofs; D.3.3 [Programming Languages]: Language Constructs and Features-Concurrent programming structures

General Terms: Languages, Theory, Verification

Additional Key Words and Phrases: Separation Logic, Concurrent Abstract Predicates, Concurrency

Acknowledgements: Dodds was supported in part by EPSRC grants EP/H010815/1, EP/H005633/1, EP/F036345. Svendsen was supported in part by the Danish Council for Independent Research project DFF - 4181-00273. Birkedal was supported in part by the ModuRes Sapere Aude Advanced Grant from The Danish Council for Independent Research for the Natural Sciences (FNU).

Author's addresses: M. Dodds, Dept. of Comp. Science, University of York, York, UK; S. Jagannathan, Dept. of Comp. Science, Purdue University, West Lafayette, USA; M. J. Parkinson, Microsoft Research Cambridge, Cambridge, UK; K. Svendsen and L. Birkedal, Dept. of Comp. Science, Aarhus University, Aarhus, Denmark.

Permission to make digital or hard copies of part or all of this work for personal or classroom use is granted without fee provided that copies are not made or distributed for profit or commercial advantage and that copies show this notice on the first page or initial screen of a display along with the full citation. Copyrights for components of this work owned by others than ACM must be honored. Abstracting with credit is permitted. To copy otherwise, to republish, to post on servers, to redistribute to lists, or to use any component of this work in other works requires prior specific permission and/or a fee. Permissions may be requested from Publications Dept., ACM, Inc., 2 Penn Plaza, Suite 701, New York, NY 10121-0701 USA, fax +1 (212) 869-0481, or permissions@acm.org.

(C) YYYY ACM 0000-0000/YYYY/01-ARTA $\$ 15.00$

DOI : http://dx.doi.org/10.1145/0000000.0000000 


\section{INTRODUCTION}

Concurrent programming is challenging because it requires programmers to parcel work into useful units, and weave suitable concurrency control to coordinate access to shared data. Coordination is generally performed by synchronisation constructs. In order that programmers can build and reason about concurrent programs, it is essential that these synchronisation constructs hide implementation details behind specifications, allowing clients to reason about correctness in terms of abstract, rather than concrete, behaviour.

For standard synchronisation constructs - e.g. locks, queues, stacks - abstract specifications are well-studied. However, many concurrent applications depend on non-standard, custom synchronisation constructs. These may have poorly-defined abstract interfaces, while at the same time depending on complex racy implementation behaviour. Verifying these constructs requires a technique that can build up strong abstractions, reason about the logical distribution of data between threads, and at the same time deal with the intricacies of low-level concurrency. This is our objective in this paper.

Our target is to verify one such custom concurrency construct: barriers used for automated parallelisation. In deterministic parallelisation, code regions in a sequential program are executed concurrently. While the parallelized program is internally nondeterministic, control constructs are used to ensure that it exhibits the same deterministic observable behaviour as its sequential counterpart. Automatic parallelisation of this kind has been well-studied for loop-intensive numerical computations. However, it is also possible to extract parallelism from irregularly structured sequential programs [Bocchino et al. 2009; Rinard and Lam 1992; Welc et al. 2005].

One way to implement deterministic parallelism is through compiler-injected barriers [Navabi et al. 2008]. We can think of these barriers as enforcing the original sequential program dependencies on shared resources. A resource could be any program variable, data structure, memory region, lock, etc. for which resource ownership guarantees are essential in order to enforce deterministic semantics. While the intuition behind using such barriers is quite simple, there are many possible implementations, and verifying that an implementation enforces the correct behaviour is challenging for several reasons:

- Custom data-structures. To enable the maximum level of parallelism, barriers are implemented using custom data-structures that collect and summarise signals.

- Non-local signalling. The patterns of signalling in a barrier implementation are highly non-local. To access a resource, a barrier must wait until all logically preceding threads have indicated that it is safe to do so. However, threads are locally unaware of this context.

- Out-of-order signalling. The parallelisation process will strive to identify the earliest point in a thread's execution path from where a resource is no longer required. In some cases, this means threads can release resources without ever acquiring them, so that subsequent signalling of this resource by its predecessor can bypass it altogether.

- Shared read access. Barriers may treat reads and writes differently to ensure preservation of sequential behaviour. Although many reads can be performed concurrently, they must be sequentialized with respect to writes. Moreover, reads must be sequentialized with respect to other reads, if there is an intervening write.

- Higher-order specifications. Abstractly, channels can be used to control access to any kind of resource for which ownership is important. Thus, the natural specification is higherorder: the resource is a parameter to the specification. Channels may control access to other channels, or even later stages of the same channel.

In this paper, we show how to tackle these verification challenges. We use impredicative concurrent abstract predicates (iCAP), a recent program logic that enables abstract, higherorder reasoning about concurrent libraries [Svendsen and Birkedal 2014a]. This allows us to reason about both high-level properties and low-level implementation details. iCAP sup- 
ports fine-grained reasoning about concurrent behaviour, meaning that each thread can be permitted exactly the behaviour it needs. Furthermore, reasoning in iCAP is local, meaning even shared state can be encapsulated and abstracted.

The result of our work is a verified high-level specification for barriers, independent of their low-level implementation. Using iCAP, we have proved that three very different lowlevel implementations satisfy the same high-level specification. In the presence of runtime thread creation and dynamic (heap-allocated) data, our specification must be both generic and dynamic, since it must be possible to construct barriers at runtime that control access to arbitrary resources. To allow this, we use iCAP's higher-order quantification mechanism to encode complex patterns of resource redistribution. It is worth emphasising that the barriers we look at were not designed with verification in mind; we have developed the specification to suit the application, not vice versa.

In this paper we focus on just the verification problem for barriers, but in a companion paper, we define a parallelising program analysis which injects appropriate barriers [Botinčan et al. 2013]. Our work here contributes to the eventual goal of a fully specified and verified system for deterministic parallelism. More generally, access to concurrent data is often controlled by custom synchronisation constructs, and our work in this paper demonstrates how to reason soundly about such bespoke concurrency constructs.

\section{Contribution}

This paper substantially revises and expands our conference paper [Dodds et al. 2011]. Our main contributions relative to [Dodds et al. 2011] are as follows:

- A revised higher-order abstract specification for the custom synchronisation barriers used in deterministic parallelism. Our new specification is cleaner and more general.

- New proofs of this specification for simplified, out-of-order and summarising barrier implementations, written using the iCAP proof system [Svendsen and Birkedal 2014a]. The first two implementations were proved correct in [Dodds et al. 2011], while the proof of the summarising version is entirely novel.

- A self-contained presentation of the formal iCAP proof system and a tutorial on how to use iCAP to verify concurrent software. The formal iCAP proof system is not presented nor explained in the iCAP conference paper [Svendsen and Birkedal 2014a].

- An encoding in iCAP of constructs we call saved propositions. These serve some of the functions of auxiliary variables capable of storing propositions, and allow us to reason about resource transfer and splitting without altering the proof system.

- A new application of Wickerson et al.'s explicit stabilization [Wickerson et al. 2010] to reason about the stability of complex separation logic assertions.

- A verified application of our barriers: a tree-based key-value store. This demonstrates a non-trivial dynamic pattern of signalling, as signals are activated at branches only if they are visited by a thread. It also demonstrates the need for higher-order reasoning: in the logical definition of a tree, the induction passes through a higher-order parameter.

This paper also corrects a subtle logical problem in [Dodds et al. 2011], discovered by Svendsen a year after publication. This arose as a result of a circularity in the model of higher-order propositions, which rendered several important reasoning steps unsound. The details are discussed in $\S 8$, but we emphasise that this problem could not have been solved by the higher-order separation logics available in 2011. The development of iCAP was in part motivated by resolving this kind of problem; in this paper, we show that iCAP can be used to verify tricky practical algorithms.

\section{Paper Structure}

$\S 2$ discusses related work. $\S 3$ introduces the behaviour of barriers informally, and defines our abstract specification. It also discusses an example application, a tree-based key-value store. 
$\S 4$ gives a very simple barrier implementation, and shows how it can be verified with respect to the core of the specification. This section also serves as a tutorial introduction to iCAP, the logic we use for verification. $\S 5$ discusses how the specification can be extended to cover the splitting of resources offered by a channel. $\S 6$ gives a more complicated implementation where channels are arranged into chains, and verifies the full abstract specification. $\S 7$ gives an optimised implementation where signals between channels are summarised, and verifies it. $\S 8$ explores the problems with our conference paper [Dodds et al. 2011], and how we have addressed them. Some of the subsidiary lemmas are proved in full in the appendices.

\section{RELATED WORK}

iCAP is a new logic for verifying complicated concurrent algorithms [Svendsen and Birkedal 2014a; 2014b]. Although we have focussed in this paper on barriers used for deterministic parallelism [Welc et al. 2005; Berger et al. 2010; Bocchino et al. 2009; Navabi et al. 2008], our intention is to illustrate how iCAP can be used to specify and verify novel concurrency constructs in general.

Prior to 2011, most work on concurrent separation logic considered concurrency constructs as primitive in the logic. This begins with O'Hearn's work on concurrent separation logic [O'Hearn 2007], which takes statically allocated locks as a primitive. CSL has been extended to deal with dynamically-allocated locks [Gotsman et al. 2007; Hobor et al. 2008; Jacobs and Piessens 2009] and re-entrant locks [Haack et al. 2008]. Others have extended separation logic or similar logics with primitive channels [Hoare and O'Hearn 2008; Bell et al. 2009; Villard et al. 2010; Leino et al. 2010], and event driven programs [Krishnaswami et al. 2010]. There are important disadvantages to handling each distinct concurrency construct with a new custom logic:

- Developing a custom logic might be acceptable for standard synchronisation constructs such as locks, but it is infeasible for every domain-specific construct.

- Embedding each construct as primitive in the logic provides no means for verifying implementations of the construct.

- Each custom logic handles one fixed kind of construct, with no means of verifying programs that use multiple concurrency constructs.

iCAP solves all three problems. New synchronisation constructs can be introduced as libraries and given abstract specifications that abstract over the internal data representation and state through abstract predicates. Implementations can be verified against these abstract specifications by giving these predicates concrete definitions (our paper does precisely this for barriers). As new constructs can be freely introduced as libraries, clients are free to combine multiple concurrency constructs as needed. Furthermore, using iCAP's higher-order quantification, specifications can abstract over arbitrary predicates, including those defined by other concurrency constructs. This allows us to support separate reasoning about each construct, while still allowing them to interact cleanly. For instance, abstract lock predicates defined by a lock library can freely be transferred through our channels. (See [Dinsdale-Young et al. 2010] for an example of such a lock specification).

iCAP descends from our earlier Concurrent Abstract Predicates (CAP) logic [DinsdaleYoung et al. 2010]. CAP combined the explicit treatment of concurrent interference from rely-guarantee [Jones 1983; Feng et al. 2007; Vafeiadis 2007] and abstraction through abstract predicates [Parkinson and Bierman 2005], with a rich system of protocols based on capabilities [Dodds et al. 2009]. iCAP extends CAP with higher-order propositions and an improved system of concurrent protocols [Svendsen and Birkedal 2014a]. iCAP's stepindexed semantics is supported by an underlying theory called the topos of trees [Birkedal et al. 2012].

Recent years have seen a great deal of work on concurrent logics, many of which take inspiration from CAP. Complex concurrency constructs have been verified before in CAP-like 
logics, e.g. concurrent B-trees in [da Rocha Pinto et al. 2011]. The proof in [da Rocha Pinto et al. 2011] is mostly concerned with complex manipulations of the B-tree structure. In comparison, our barrier implementations are relatively simple, and a large proportion of our proof concerns changes in ownership to support our higher-order specification. The verification of the Joins library in [Svendsen et al. 2013] has similarities to our work. Both papers deal with barriers using higher-order separation logic. However, the implementations and specifications are substantially different - for example our implementation permits chains of channels, and our specification deals with resource-splitting. The authors of [Svendsen et al. 2013] are also co-authors on this paper, and iCAP was largely developed as a improvement on the HOCAP logic introduced there.

Two significant alternative logics to iCAP are CaReSL [Turon et al. 2013] and TaDA [da Rocha Pinto et al. 2014]. Like iCAP, both extend CAP with richer protocols. Unlike iCAP, both are primarily aimed at proving atomicity / linearizability, and confine themselves to second-order logic only. This makes them less suitable for our purposes. It is plausible that many of the proofs in this paper could be recast into these logics. However, we would have to constrain the higher-order parameters from our specification with some kind of explicit stratification. We would expect proofs to be significantly more complex as a result of the bookkeeping needed to track this stratification.

Another logic aimed at fine-grained concurrent data-structures is FSCL [?]. This is defined through a shallow embedding into Coq's Calculus of Inductive Constructions, and thus supports definition of higher-order specifications. However, FCSLs reasoning principles for higher-order specifications are weaker than those of iCAP. In particular, FCSL lacks support for impredicative protocols and assertions in the heap, both of which result in circularities that would manifest as universe inconsistency errors in Coq. Thus, while FCSL can define our proposed barrier specification, we believe that FCSL would be unable to verify an implementation against it. Impredicative protocols and assertions in the heap are fundamental to the generic higher-order specifications that we require. For example, in $\S 3.6$, we apply our barrier specification in a key-value store: here the inductive definition of a tree passes through a higher-order parameter, representing the fact that subtrees must be acquired through channel communication.

\section{A SPECIFICATION FOR DETERMINISTIC PARALLELISM}

In this section, we describe the intuitive behaviour of a library of barriers for enforcing deterministic parallelism that forms our case study. Based on this, we define a high-level specification for barriers - the full abstract specification is given in $\S 3.4$. These barriers are based on the ones used for deterministic parallelism in [Navabi et al. 2008]. In [Botinčan et al. 2013] we use our abstract specification in a proof-based parallelizing analysis that is guaranteed to preserve sequential behaviour.

We assume that code sections believed to be amenable for parallelization have been identified, and the program split accordingly into threads. We assume a total logical ordering on threads, such that executing the threads serially in the logical order gives the same result as the original (unparallelized) program.

Barriers are associated with resources (e.g., program variables, data structures, etc.) that are to be shared between concurrently-executing program segments. There are two sorts of barriers. A signal barrier notifies logically later threads that the current thread will no longer use the resource. A wait barrier blocks until all logically prior threads have signalled that they will no longer use the resource (i.e., have issued signals).

We assume barriers are injected by an analysis which ensures that all salient data dependencies in the sequential program are respected. For example, suppose we run two instances of the function $f$ in sequence (here sleep(rand () ) waits for an unknown period of time). 
We will reason about $\mathrm{f} 1$ and $\mathrm{f} 2$ using separation logic, which lets us precisely control the allocation of resources to threads over time. Assertions in separation logic denote resources: heap cells and data-structures, but also abstract resources like channel ends. For example, we write the following assertion to denote that $\mathrm{x}$ points to value $v$ and $\mathrm{y}$ to value $v^{\prime}$ : $\mathrm{x} \mapsto v * \mathrm{y} \mapsto v^{\prime}$ The separating conjunction $*$ asserts that $\mathrm{x}$ and $\mathrm{y}$ are distinct. As well as capturing information about the current state of resources, assertions in separation logic also capture ownership. Thus the assertion $\mathrm{x} \mapsto v * \mathrm{y} \mapsto v^{\prime}$ in an invariant for a thread implicitly states that the thread has exclusive access to $\mathrm{x}$ and $\mathrm{y}$.

To reason about the parallel composition of threads, we can use the PAR rule of concurrent separation logic [O'Hearn 2007]:

$$
\frac{\left\{P_{1}\right\} C_{1}\left\{Q_{1}\right\} \quad\left\{P_{2}\right\} C_{2}\left\{Q_{2}\right\}}{\left\{P_{1} * P_{2}\right\} C_{1} \| C_{2}\left\{Q_{1} * Q_{2}\right\}} \text { PAR }
$$

To verify $f 1$ and $f 2$, we must encode the fact that $f 1$ gives up access to $x$ and y by calling signal (i), while $f 2$ retrieves access to them by calling wait(i). We encode these two facts with two predicates, recv and send, corresponding to the promised resource, the resource that can be acquired from logically earlier threads, and the required resource, the resource that must be supplied to logically later threads. We read these as follows:

$$
\begin{aligned}
\operatorname{recv}(i, P)- & \text { By calling wait on } i \text {, the thread will acquire a resource satisfying } \\
& \text { the assertion } P . \\
\text { send }(i, P)- & \text { By calling signal on } i \text { when holding a resource satisfying } P \text {, the } \\
& \text { thread will lose the resource } P .
\end{aligned}
$$

These predicates are abstract; each instantiation of the library will define them differently. The client only depends on an abstract specification that captures their intuitive meaning:

$$
\begin{array}{rcl}
\{\operatorname{emp}\} & i=\operatorname{newchan}() & \{\operatorname{send}(i, P) * \operatorname{recv}(i, P)\} \\
\{\operatorname{send}(i, P) * \operatorname{stable}(P) * P\} & \operatorname{signal}(i) & \{\operatorname{emp}\} \\
\{\operatorname{recv}(i, P) * \operatorname{stable}(P)\} & \operatorname{wait}(i) & \{P\}
\end{array}
$$

The assigned variable i stands for newchan's return value - i.e. the address of the new channel. We also use this notation in the specification of extend, below.

This specification of newchan is implicitly universally quantified for all assertions $P$, meaning that we can construct a channel for any iCAP assertion. The same is true for other operation specifications: unless otherwise stated, predicates are universally quantified and thus can be chosen freely.

In general, the universally quantified $P$ can be instantiated with assertions about shared resources. In this case we need to establish that these assertions are stable, i.e., invariant under changes potentially performed by other threads. This is expressed by the stability assertion, stable $(P)$, in the preconditions of signal and wait. Stability will be explained in §4.1. For the moment, note that if $P$ is a thread-local assertion, such as $x \mapsto v$, then $P$ is trivially stable, as these assertions assert exclusive ownership of the underlying resource.

Note that we do not require stability in the specification of newchan - i.e. the proposition $P$ in $\{\operatorname{send}(i, P) * \operatorname{recv}(i, P)\}$ need not be invariant. This gives us more freedom, because $P$ can be be dynamically modified before a corresponding resource is supplied - see the sections below on splitting and renunciation. We only require that when a resource is eventually supplied or recieved, it is stable.

New recv and send predicates can be constructed at run-time using newchan, meaning we can construct an arbitrarily large number of channels for use in the program. Given these two predicates, we can give the following specifications for $f 1$ and $f 2$. (Here we specialise 
To support splitting, we add a property to the specification allowing threads to divide promised resources:

$$
\{\operatorname{recv}(a, P * Q) * \operatorname{stable}(P * Q)\} \quad\langle\operatorname{skip}\rangle \quad\{\operatorname{recv}(a, P) * \operatorname{recv}(a, Q)\}
$$

This axiom states that when a thread has been promised a resource that consists of two parts, access can be split between two threads, potentially before the resource is available. This is achieved by splitting a single promise for a resource consisting of two disjoint parts into two promises, one for each part.

Note that the splitting property is not a logical entailment - applying it requires an operational step, skip. This is because the property manipulates a shared higher-order resource: $\operatorname{recv}(a, P)$. To avoid problems with self-reference, iCAP requires that such manipulations correspond with operational steps - this anomaly is discussed when we introduce $\mathrm{iCAP}$ in $\S 4.1$. Thus we have to assume that every application of the splitting specification is associated with a skip. We discuss whether this assumption is justified in $\S 4.1$.

\subsection{Chains and renunciation}

To allow many threads to access related resources in sequence, we can construct a chain of channels. A wait barrier called on a channel waits for signal barriers on all preceding channels. We use the ordering in a chain to model the logical ordering between a sequence of parallelized threads. A chain initially consists of a singleton channel constructed using newchan. We introduce an operation extend which takes as its argument an existing channel, and creates a new channel immediately preceding it in the chain.

Connecting channels into chains creates a new opportunity for parallelism: the ability to renounce access to a resource without acquiring it first. In the simple specification given above, a thread can only call signal if it has acquired the required resource from its predecessors. However, this is often unnecessary. For example, consider the following three threads:

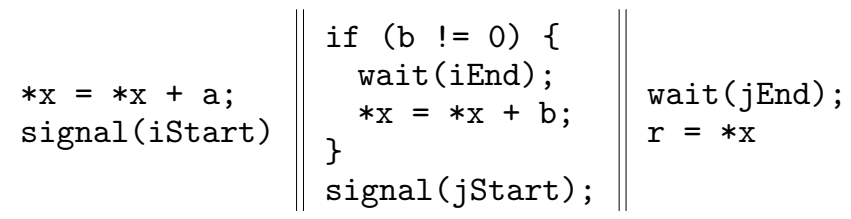

Here we give the start and end points of channels distinct names: iStart and iEnd are the start and end points of channel $i$ (resp. $j$ ). The channels $i$ and $j$ are arranged in a chain. The second thread waits on iEnd only if it needs to access x. Otherwise it signals immediately, even if the first thread has not signalled. Without renunciation the thread would have to insert a wait confirming that the first thread had signalled.

Chains. To support chains in our specification, we introduce an order predicate '_ $\prec$ ' which represents the order between links in the chain. $x \prec y$ asserts that the channel $x$ is earlier in the chain than channel $y$. We use two axioms about the ordering of channels:

$$
\begin{aligned}
& x \prec y \Longrightarrow x \prec y * x \prec y \quad \text { (duplication) } \\
& x \prec y * y \prec z \Longrightarrow x \prec z \quad \text { (transitivity) }
\end{aligned}
$$

The precondition for the three-thread example above would therefore be as follows. The predicate $i$ End $\prec j$ Start represents the relationship between the two channels $i / j$ :

$$
\left\{\begin{array}{l}
\operatorname{send}\left(\text { iStart }, x \mapsto \mapsto_{-}\right) * \operatorname{recv}\left(\text { iEnd, } x \mapsto_{-}\right) * \\
\operatorname{send}\left(j \operatorname{Start}, x \mapsto_{-}\right) * \operatorname{recv}\left(j \text { End, } x \mapsto_{-}\right) * \text { iEnd } \prec \text { jStart }
\end{array}\right\}
$$


The abstract specification of extend takes a send predicate and a set of order predicates about earlier channels $E$, and a set of order predicates about later channels $L$. The function returns a pair of channels $(\mathrm{a}, \mathrm{b})$, that are later than all the channels before $\mathrm{x}$ and before all the channels after $\mathrm{x}$, and $\mathrm{a}$ is before $\mathrm{b}$ in the chain. Note this means a single channel's start and end-point may have different identifiers. It also creates recv, send, and order predicates representing the new channel.

$$
\left\{\begin{array}{l}
\operatorname{send}(\mathrm{x}, P) * \\
\circledast_{e \in E} e \prec \mathrm{x} * \circledast_{l \in L} \mathrm{x} \prec l
\end{array}\right\} \quad(\mathrm{a}, \mathrm{b})=\operatorname{extend}(\mathrm{x}) \quad\left\{\begin{array}{l}
\operatorname{send}(\mathrm{a}, Q) * \operatorname{recv}(\mathrm{a}, Q) * \operatorname{send}(\mathrm{b}, P) \\
* \mathrm{a} \prec \mathrm{b} * \circledast_{e \in E} e \prec \mathrm{a} * \circledast_{l \in L} \mathrm{~b} \prec l l
\end{array}\right\}
$$

Using this, we can construct the precondition for the three-thread example as follows:

$$
\begin{aligned}
& \{\operatorname{emp}\} \\
& \text { jEnd }=\operatorname{newchan}() ; \\
& \left\{\operatorname{send}\left(j \operatorname{End}, x \mapsto_{-}\right) * \operatorname{recv}\left(j \operatorname{End}, x \mapsto_{-}\right)\right\} \\
& (\text {iEnd }, j \operatorname{Start})=\operatorname{extend}(j \text { End }) ; \\
& \text { iStart }=\text { iEnd; } \\
& \left\{\begin{array}{l}
\operatorname{send}\left(i \operatorname{Start}, x \mapsto_{-}\right) * \operatorname{recv}\left(i \text { End }, x \mapsto_{-}\right) * \\
\operatorname{send}\left(j \operatorname{Start}, x \mapsto_{-}\right) * \operatorname{recv}\left(j \text { End }, x \mapsto_{-}\right) * \text { iEnd } \prec \text { jStart }
\end{array}\right\}
\end{aligned}
$$

Renunciation. To support renunciation, we add an axiom allowing threads to satisfy required resources using earlier promised resources:

$$
\{\operatorname{recv}(x, P) * \operatorname{send}(y, P * Q) * x \prec y\} \quad\langle\operatorname{skip}\rangle \quad\{\operatorname{send}(y, Q)\}
$$

By giving up the ability to acquire the $P$ resource on the $x$ channel, we can forward the $P$ resource to partially discharge our send obligation on a subsequent channel $y$. If the initial send obligation on $y$ requires us to supply a resource with two disjoint parts $P$ and $Q$, after renouncing $P$ to $y$, the obligation on $y$ reduces to $Q$.

In the three-thread example, we can use this specification to justify signalling on jStart without waiting on iEnd:

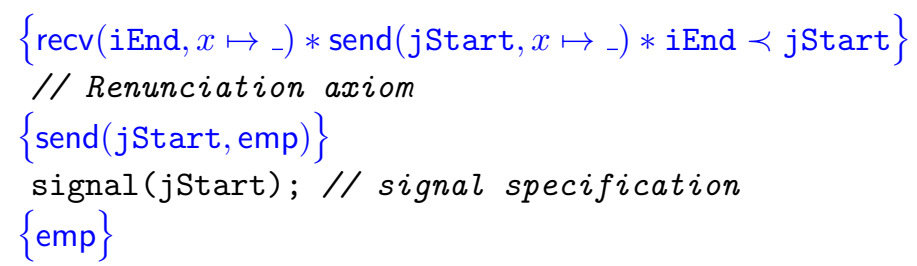

\subsection{Full abstract specification}

Figure 2 shows our client-facing abstract specification for deterministic parallelism. It introduces the extra predicates and axioms to support chains, renunciation and splitting.

Note that in $\S 5$ we define a more general specification which is more convenient when verifying channel implementations. Specifically, it uses explicit stabilization rather than stable assertions (see $§ 4.1$ ). The client-facing specification given in Figure 2 is less general, but also less complex, and sufficient to verify all our examples.

\subsection{Adding Forward Extension}

The specification in Fig. 2 allows chain extension 'backwards', by inserting a channel immediately before an existing send. However, in order to impose a sequential order, it is often useful to allows extension in the other direction, at the end of the chain. Fortunately, it is 


$$
\begin{aligned}
& \text { Specifications: } \\
& \{\text { emp }\} \quad i=\operatorname{newchan}() \quad\{\operatorname{recv}(i, P) * \operatorname{send}(i, P)\} \\
& \{\operatorname{send}(i, P) * \operatorname{stable}(P) * P\} \quad \operatorname{signal}(i) \quad\{\text { emp }\} \\
& \{\operatorname{recv}(\mathrm{i}, P) * \operatorname{stable}(\mathrm{P})\} \quad \text { wait }(\mathrm{i}) \quad\{P\} \\
& \left\{\begin{array}{l}
\operatorname{send}(\mathrm{x}, P) * \\
\circledast_{e \in E} e \prec \mathrm{x} * \circledast_{l \in L} \mathrm{x} \prec l
\end{array}\right\} \quad(\mathrm{b}, \mathrm{a})=\operatorname{extend}(\mathrm{x})\left\{\begin{array}{l}
\operatorname{send}(\mathrm{b}, Q) * \operatorname{recv}(\mathrm{b}, Q) * \operatorname{send}(\mathrm{a}, P) \\
* \mathrm{~b} \prec \mathrm{a} * \circledast_{e \in E} e \prec \mathrm{b} * \circledast_{l \in L} \mathrm{a} \prec l
\end{array}\right\} \\
& \text { Axioms: } \quad x \prec y \quad \Longrightarrow \quad x \prec y * x \prec y \\
& x \prec y * y \prec z \quad \Longrightarrow \quad x \prec z \\
& \{\operatorname{recv}(x, P) * \operatorname{send}(y, P * Q) * x \prec y\} \quad\langle\operatorname{skip}\rangle \quad\{\operatorname{send}(y, Q)\} \\
& \{\operatorname{recv}(a, P * Q) * \operatorname{stable}(P * Q)\} \quad\langle\operatorname{skip}\rangle \quad\{\operatorname{recv}(a, P) * \operatorname{recv}(a, Q)\}
\end{aligned}
$$

Fig. 2. Abstract specification for deterministic parallelism.

simple to implement a wrapper library with this behaviour on top of our abstract specification. We use an object called seqChan to represent the end of the chain, with specification as follows:

$$
\begin{aligned}
& \{\operatorname{seq} C h a n(\mathrm{x}, P)\} \mathrm{cw}, \mathrm{cs}=\operatorname{extendSC}(\mathrm{x})\{\operatorname{recv}(\mathrm{cw}, P) * \operatorname{send}(\mathrm{cs}, Q) * \mathrm{cw} \prec \mathrm{cs} * \operatorname{seq} C h a n(\mathrm{x}, Q)\} \\
& \{P\} \mathrm{sc}=\text { newSeqChan }() \quad\{\operatorname{seq} \operatorname{Chan}(\mathrm{sc}, P)\}
\end{aligned}
$$

To implement a seqChan object, we store a pair of channels. The first, cWait, is the recv point for the resource $P$. The second, cEnd is a dummy send channel used to allow extension. In order to extend, the wrapper library calls extend (cEnd) - this yields in a new channel that can replace cWait. The seqChan object is thus defined as follows:

$\operatorname{seq} C h a n(\mathrm{x}, P) \triangleq \exists c w, c e . \mathrm{x} . \mathrm{cWait} \mapsto c w * \mathrm{x} . \mathrm{cEnd} \mapsto c e * \operatorname{recv}(c w, P) * \operatorname{send}\left(c e,,_{-}\right) * c w \prec c e$

The details of the implementation and verification of the wrapper library are given in Fig. 3. Note that all the reasoning in this proof uses the abstract specification in Fig. 2 - we can use this specification to build and verify richer families of synchronisation constructs.

\subsection{Example: Concurrent Key-value Store}

We will now use our channels to implement a simple tree-based key-value store. We will focus on two methods: an add/update method which inserts a key-value pair, and a reverse lookup method which returns the key associated with a particular value, if it exists. These methods are called sequentially, but our implementation permit concurrent updates and lookups internally within the data-structure. The methods have the following specification:

$$
\begin{array}{lll}
\{\operatorname{Tree}(\mathrm{x}, m)\} \quad \operatorname{add}(\mathrm{x}, \mathrm{k}, \mathrm{v}) ; & \{\operatorname{Tree}(\mathrm{x}, m[\mathrm{k} \mapsto \mathrm{v}])\} \\
\{\operatorname{Tree}(\mathrm{x}, m)\} \mathrm{k}=\operatorname{revlookup}(\mathrm{x}, \mathrm{v}) ; & \{\operatorname{Tree}(\mathrm{x}, m) *(m(\mathrm{k})=\mathrm{v} \vee(\mathrm{k}=-1 \wedge \mathrm{v} \notin i m g(m)))\}
\end{array}
$$

The second parameter of the Tree predicate represents the contents of the key-value store, i.e. it is a partial function $m$ : Key $\rightarrow$ Val.

This specification requires that methods are called sequentially, and indeed makes it appear that the each method runs entirely sequentially. For example, the postcondition of $\operatorname{add}(\mathrm{x}, \mathrm{k}, \mathrm{v})$ is a Tree updated so that the store maps key $\mathrm{k}$ to value v. However, internally the add operation forks a new thread to apply the update, then immediately returns. In other words, internally add and revlookup operations run concurrently, but our channels enforce the illusion of sequential access. 


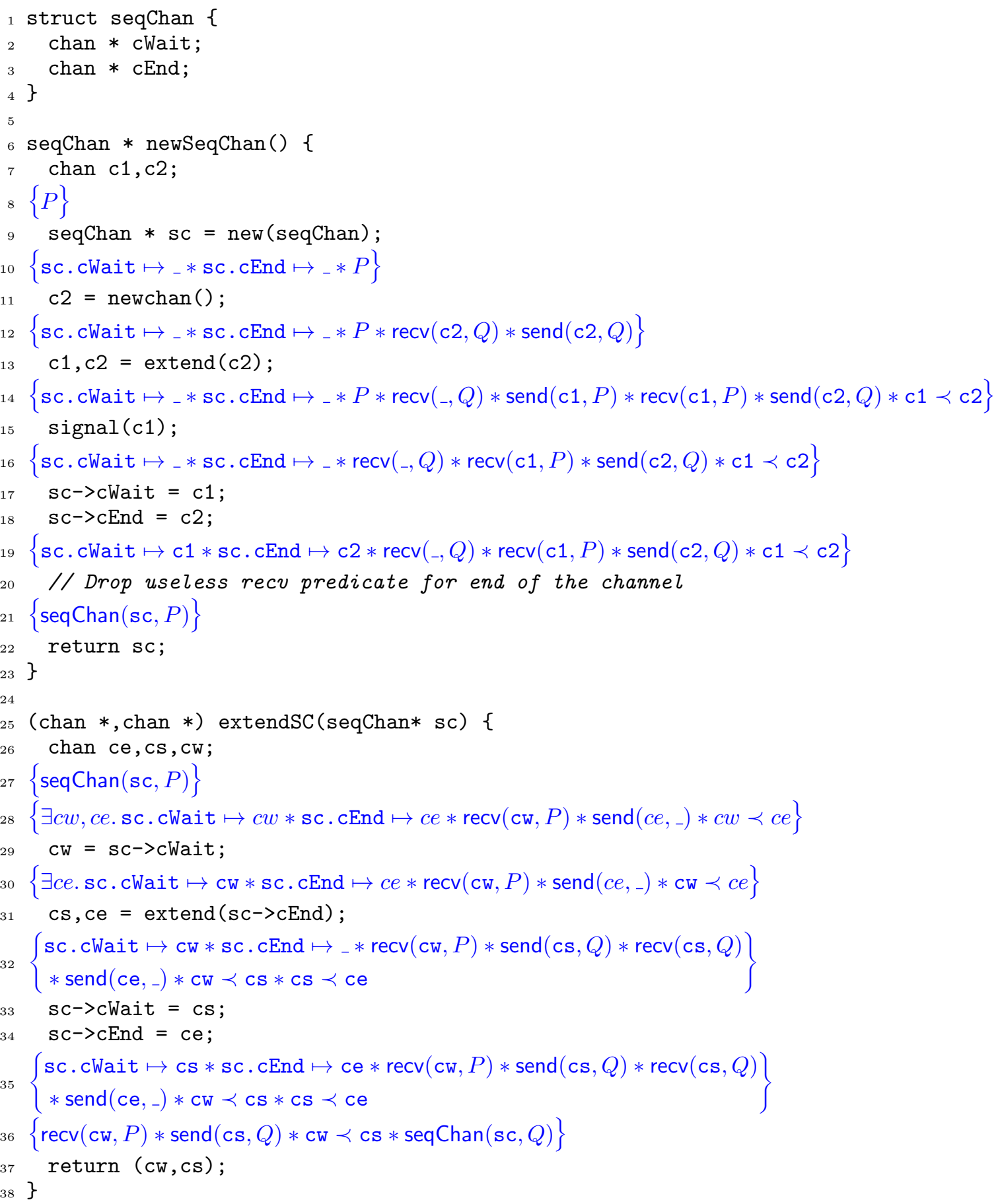

Fig. 3. Source code and proof outlines for seq channels 


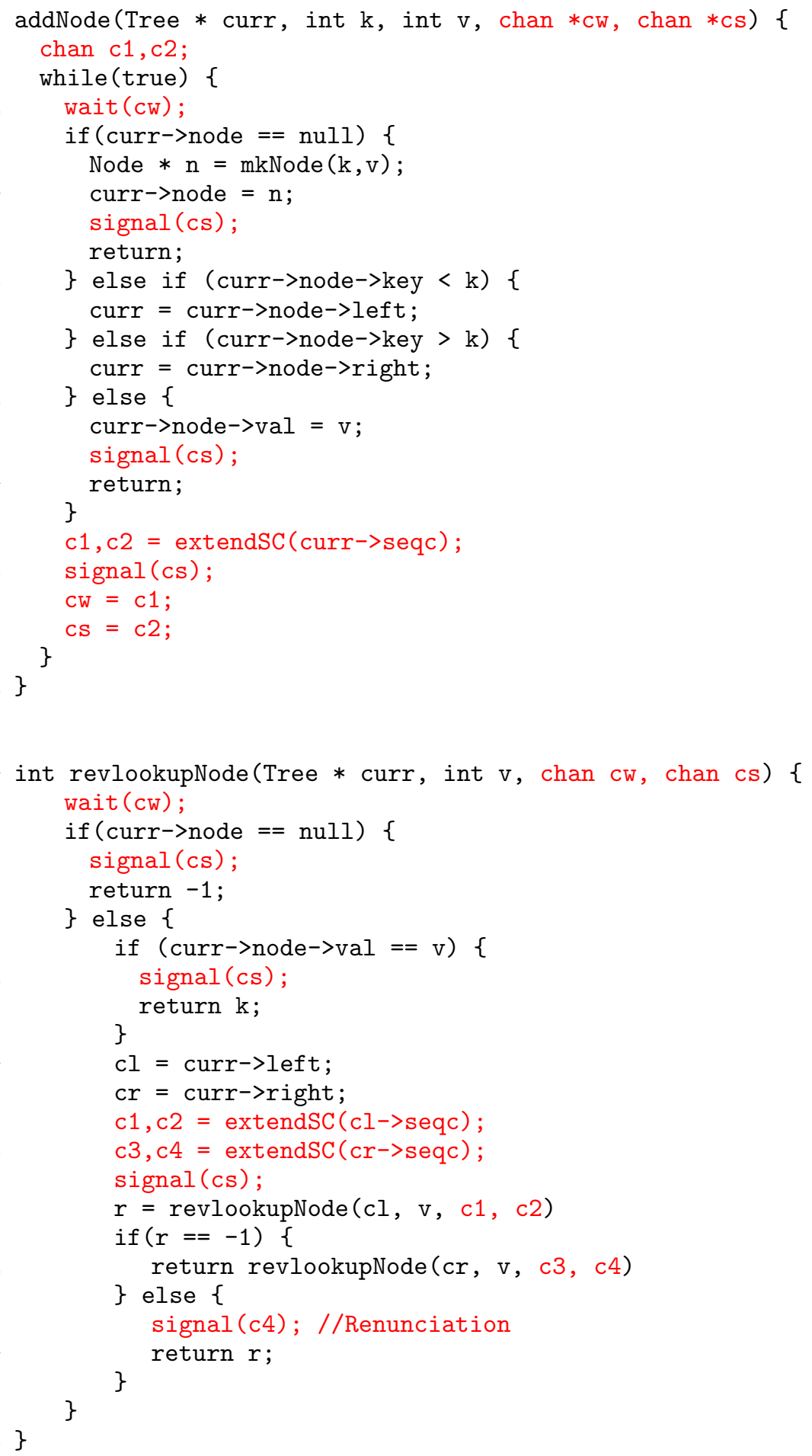

Fig. 4. Concurrent helper methods for the key-value store. 
revlookupNode follows a similar pattern, but an operation registers its presence in both left and right subtrees, because it may need to search both (lines 22-23). If the value is located in the left subtree, then the right subtree will never be searched, and can be signalled without waiting (line 42) - in other words, this is an example of renunciation.

Note that the need to wait for a return value sequentialises calls to revlookup. However, this restriction could be lifted with a little more work: revlookup could return a channel identifier through which its result value could be passed. A client program could then initiate multiple calls to revlookup in sequence, and wait for all the results at once. Internally, these calls would run in parallel in precisely the same way as the current implementation. This would break the illusion of sequential execution, as clients would need to manage returnvalue channels. However, this specification could be verified with largely identical internal reasoning to the current version.

Verifying the key-value store. The predicate Tree representing the store data-structure is defined as follows:

$$
\begin{aligned}
\operatorname{Tree}(x, m) \triangleq & \exists c . \operatorname{seq} C h a n(c, \exists r . x . \text { node } \mapsto r * \operatorname{Node}(r, m)) * x . \text { seqc } \mapsto c \\
\operatorname{Node}(x, m) \triangleq & \exists l, r, k . x . \text { key } \mapsto k * x . \operatorname{val} \mapsto v * x . \text { left } \mapsto l * x . \text { right } \mapsto r * \\
& \operatorname{Tree}\left(l, m_{l}\right) * \operatorname{Tree}\left(r, m_{r}\right) * m_{l} ;[k: v] ; m_{r}=m * k e y s\left(m_{l}\right)<k<k e y s\left(m_{r}\right) \\
\operatorname{Node}(\operatorname{null}, m) \triangleq & m=\emptyset
\end{aligned}
$$

The Tree predicate consists of a pointer to a seqChan predicate, which promises the rest of the tree. Thus, by using extendSC and wait, a thread can gain access to the remainder of the tree. The Node predicate splits the map $m$ between its key/value, and the left and right subtrees. Each subtree is represented recursively by a Tree predicate.

The key step in the proof lifts the specification of extendSC to the Tree predicate. The correctness of this step follows trivially from the definition of Tree.

$$
\begin{aligned}
& \{\operatorname{Tree}(\mathrm{t}, m)\} \\
& \text { cw, cs }=\operatorname{extendSC}(\mathrm{t}->\text { seqc }) ; \\
& \left\{\begin{array}{l}
\operatorname{Tree}\left(\mathrm{t}, m^{\prime}\right) * \operatorname{recv}(\mathrm{cw}, \exists x . \operatorname{curr} . \operatorname{node} \mapsto x * \operatorname{Node}(x, m)) \\
* \operatorname{send}\left(\mathrm{cs}, \exists x . \operatorname{curr} . \operatorname{node} \mapsto x * \operatorname{Node}\left(x, m^{\prime}\right)\right) * \mathrm{cw} \prec \mathrm{cs}
\end{array}\right\}
\end{aligned}
$$

In the precondition, the current thread is promised a tree containing map $m$. This proof step promises that $m$ will then be updated to some other map $m^{\prime}$. This $m^{\prime}$ can be chosen as needed to represent the correct update - the choice is entirely arbitrary. However, before the channel can be signalled, the channel specification requires that the map $m^{\prime}$ is established, which enforces the obligation to make a reasonable choice. This step also gives a handle to the old promise, cw, and a handle used to establish the new promise, cs.

This proof step is used in the add and addNode functions. For example, in add, the promised map is updated to $m[k: v]$ as follows. This guarantees a correctly-updated datastructure to the next thread accessing the store, but defers the actual data-structure modification.

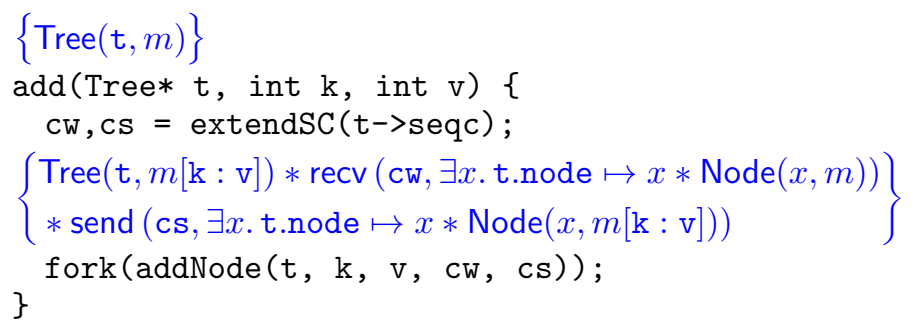


$\{\operatorname{Tree}(\mathrm{t}, m[\mathrm{k}: \mathrm{v}])\}$

The proof outline for addNode can be found in Fig. 5. Each iteration of the main loop updates one subtree. The loop invariant (line 6) consists of a recv predicate for the previous version of the subtree, and a send predicate for the subtree updated with the key/value pair. Calling wait grants access to the previous version of the subtree. The algorithm then examines the node contents and branches on the results.

If the current node is null, then the map $m$ must be empty. Therefore, send can be satisfied by just creating a single node containing the key/value pair (line 14). The case where the node already exists is similar.

In the recursive case, the algorithm first promises to update the tree by calling extendSC on the appropriate subtree (line 19). It then uses signal to indicate it has finished with the current node, and then recurses to the appropriate subtree.

More specifically, suppose the algorithm takes the left branch. In order to signal, it must first satisfy the send on cs, which requires a Node predicate with the key/value pair inserted. It is easy to prove that the map can be partitioned into subtrees less-than and greater-than the current key - these two maps are written $m_{l}$ and $m_{r}$ (line 22). As the required key must be added to the left subtree, the right subtree need not be updated. Calling extendSC promises to updates the left subtree with the key/value pair, which in turn satisfies the Node predicate required by send. This means the algorithm can call signal (line 23).

The proof structure for revlookup is similar to the one for add: by extending the chain with extendSC, the program gains the right to access the tree structure, while still permitting other threads to work on the tree at the same time.

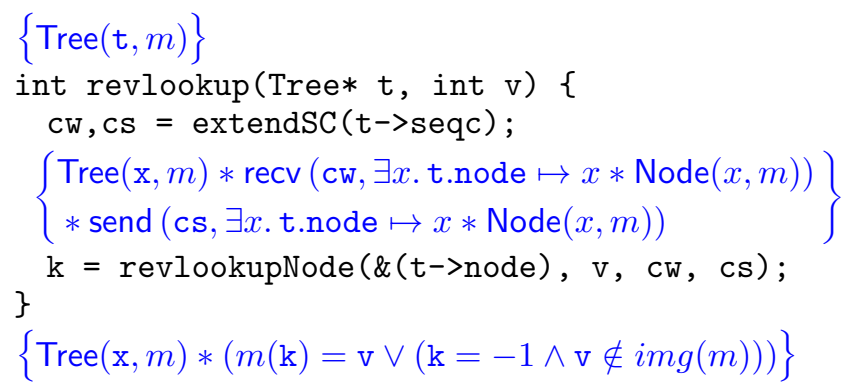

The proof outline for the revlookupNode helper function can be found in Figures 6 and 7 . The broad structure of the reasoning is similar to addNode, albeit expressed inductively rather than iteratively. Each step down the tree retrieves a subtree by calling wait, and then releases the portion of the tree it has already searched using signal. Because subtrees are associated with channels, the algorithm only accesses the portion of the tree it needs, leaving the remainder of the tree available to other threads.

One significant step is the renunciation that takes place on line 40. Here the algorithm takes requirement to supply a subtree to $c 4$ and satisfies it using the recv predicate for $c 3$. In other words, the resource is released without ever being acquired by the thread.

Summary. Thus, using our abstract specification for channels, we have verified the behaviour of the parallelised key-value store. Crucially, even though this program features many threads running at once, with complex communication between threads, each individual thread is able to reason locally, without dealing with other threads or the implementation of the barriers. Furthermore, we are able to verify a function specification which hides the existence of internal concurrency. In [Botinčan et al. 2013] we use the same abstract specification as the basis for a general parallelisation analysis. 


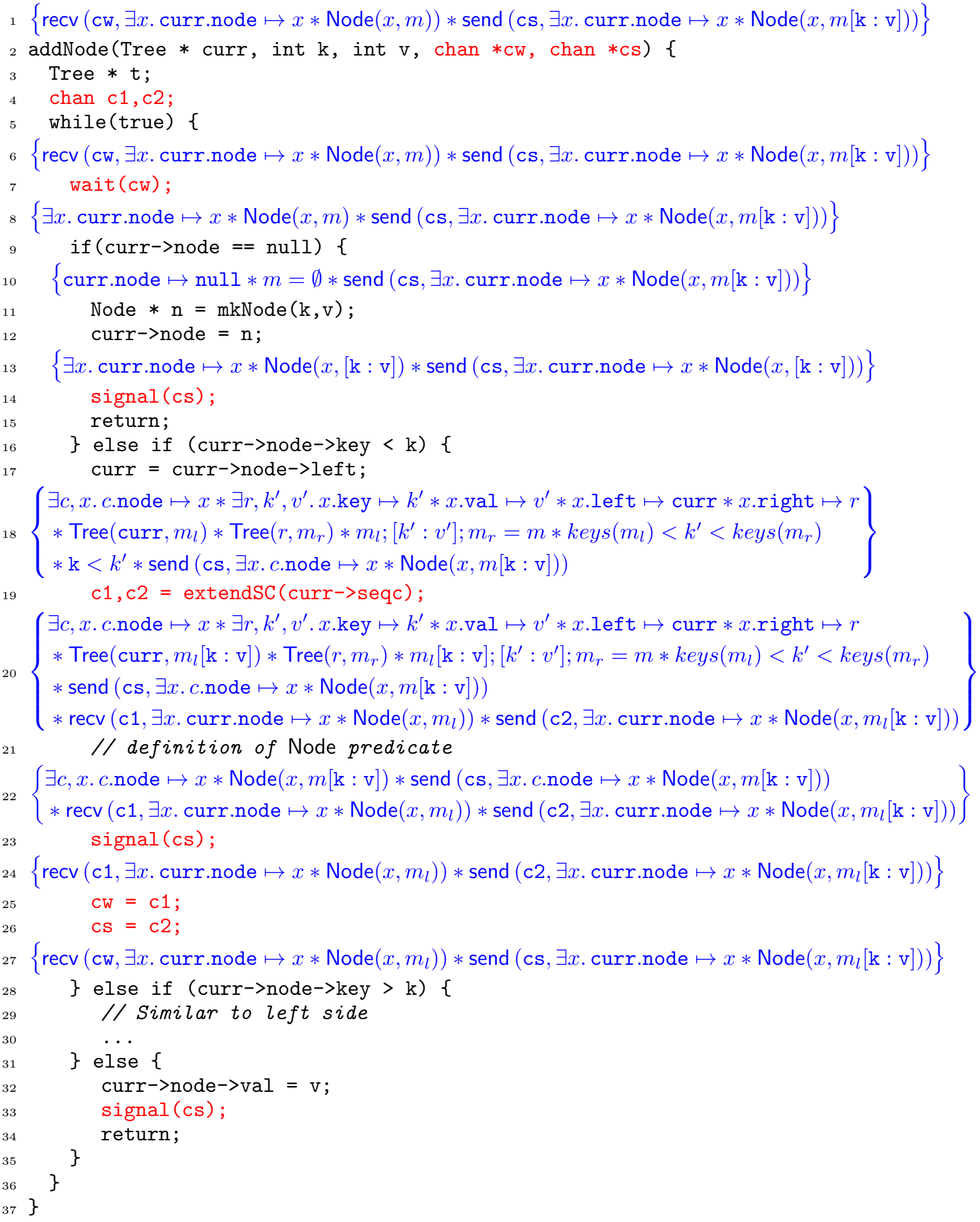

Fig. 5. Proof outline for addNode. 


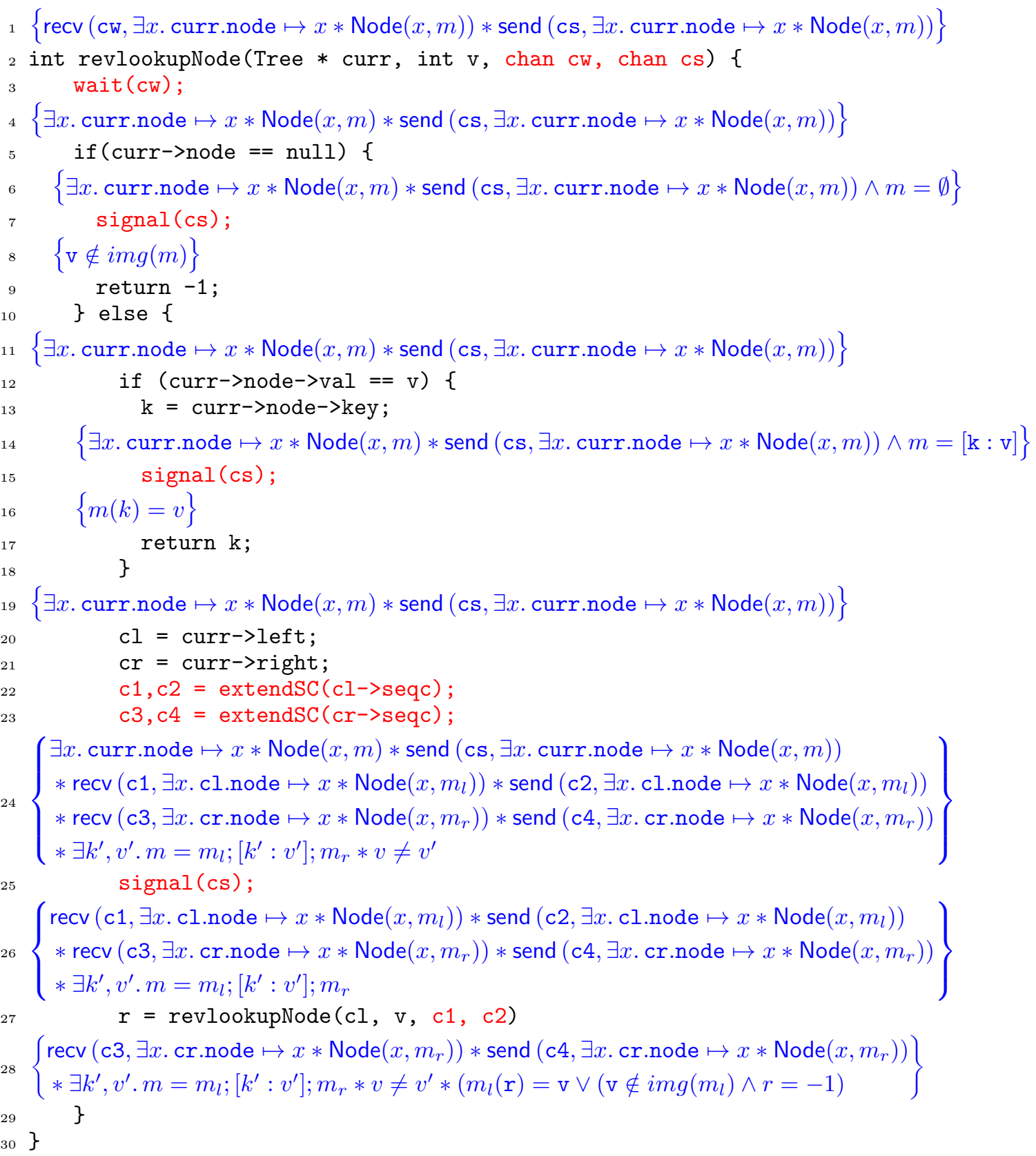

Fig. 6. Proof outline for revlookupNode (completed in Fig. 7) 


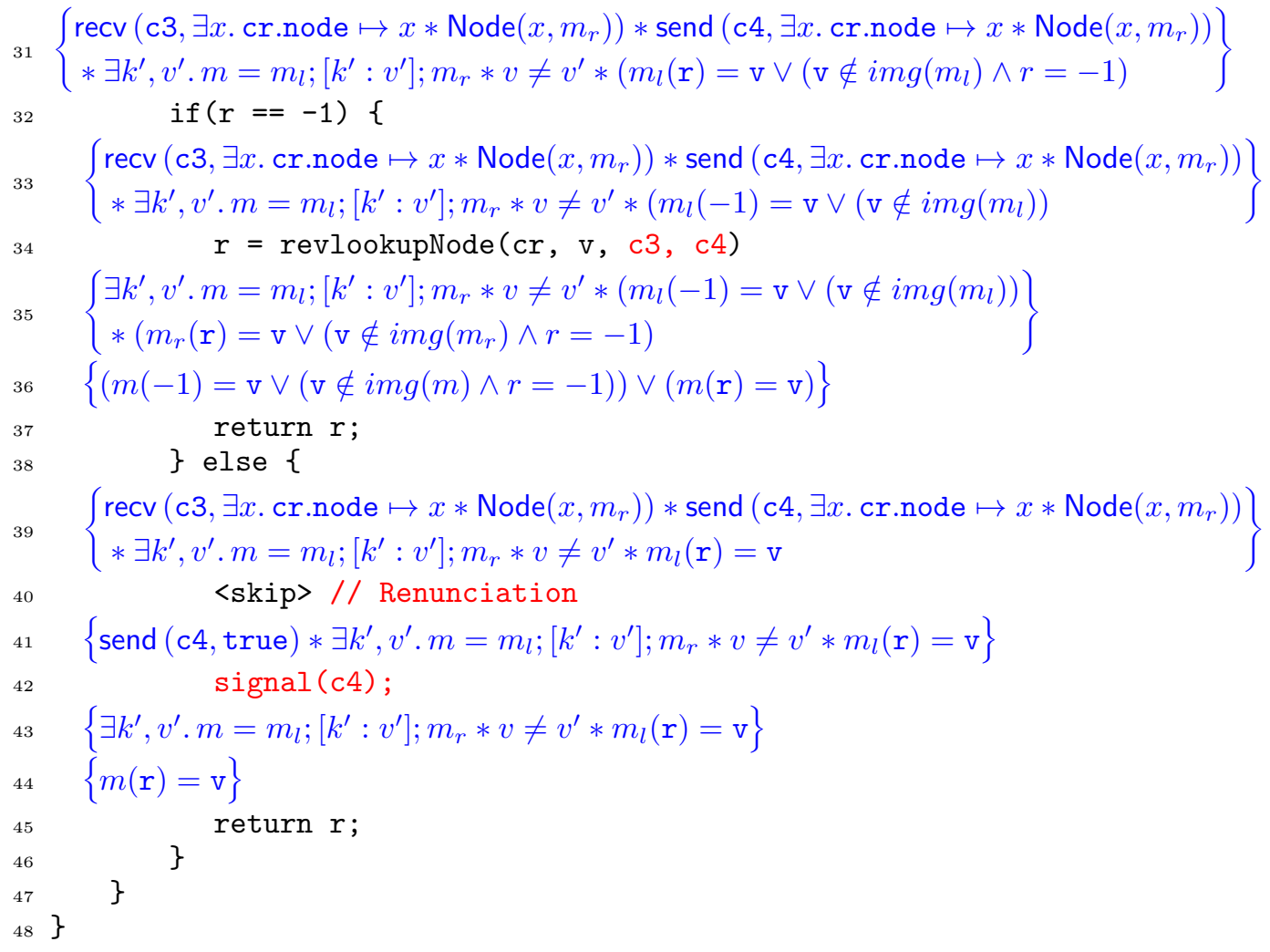

Fig. 7. Proof outline for revlookupNode (continued from Fig. 6)

\section{PROOF STRATEGY}

This section provides an intuitive introduction to our general proof approach, and to iCAP, the reasoning system our proofs are based on. We introduce iCAP using a tutorial-style presentation by verifying a simple channel implementation against a simplified barrier specification consisting of strengthened versions of the first three axioms of our abstract specification:

$$
\begin{array}{rcl}
\{\operatorname{emp}\} & i=\operatorname{newchan}() & \{\operatorname{recv}(i, P) * \operatorname{send}(i, P)\} \\
\{\operatorname{send}(i, P) * \operatorname{stable}(P) * P\} & \operatorname{signal}(i) & \{\operatorname{emp}\} \\
\{\operatorname{recv}(i, P) * \operatorname{stable}(P)\} & \text { wait }(i) & \{P\}
\end{array}
$$

By avoiding splitting, chain extension, and renunciation, we can illustrate the basic features of iCAP in a straightforward manner.

Figure 8 gives a simple barrier implementation. Each channel has a flag field representing the current state of the channel. Each send / recv pair is associated with one such structure in the heap. The signal simply sets the flag, while the wait loops until the flag is set. In $\S 5$ and $\S 6$ we reintroduce the necessary extra reasoning to verify our full abstract specification.

\section{1. iCAP tutorial}

Impredicative Concurrent Abstract Predicates (iCAP) is a separation logic variant intended for verifying concurrent higher-order programs [Svendsen and Birkedal 2014a; 2014b]. In this 
$I(x)$ describes the resources contained in the region when it is in abstract state $x \in R$. To allow multiple distinct regions, $r$ is a unique identifier for this region.

The remaining field, $T$, is a transition relation over abstract states, with transitions labelled with actions. $T$ and $I$ express the protocol that all threads must adhere to when accessing the region. Threads are only allowed to move a region from one abstract state to another if there exists a path in the labelled transition system $T$ labelled with actions permitted to the thread. Permitted actions are tracked using tokens. These are linear objects created at the same time as a region. By issuing threads different tokens, we grant them different abilities over the shared region. For instance, the following assertion asserts ownership of a set and change token: [set $]_{i}^{r_{1}} *\left[\right.$ change ${ }_{j}^{r_{2}}$. Here $r_{1}$ and $r_{2}$ are the identifiers for the associated regions, while $i$ and $j$ are fractional parameters tracking how ownership of each token is shared.

A token with full permission $(i=1)$ asserts exclusive ownership of the action and thus ensures that no other thread can use the given action to change the abstract state of the shared region. Partial permission $(i<1)$ allows the owner to use the action, but does not exclude other currently executing threads from also using the action. Tokens can be split if the permission value is preserved: $[\alpha]_{i+j}^{r} \Leftrightarrow[\alpha]_{i}^{r} *[\alpha]_{j}^{r}$. In contrast, region assertions can be duplicated arbitrarily:

$$
\operatorname{region}(R, T, I, r) \Longrightarrow \operatorname{region}(R, T, I, r) * \operatorname{region}(R, T, I, r)
$$

This allows arbitrarily many threads to access a shared region, but the ability to modify the region is restricted to threads holding the appropriate tokens.

Defining send and recv. To verify the implementation shown in Figure 8 with respect to the abstract specification we must first give concrete definitions to the abstract predicates send and recv.

The idea is to introduce a shared region for each channel, governing the internal state of that channel (the flag field) and ownership of the promised resource $P$. The shared region will have three possible abstract states: Low, High and Done. In the Low state the flag is low and the promised resource has yet to be provided. In the High state the flag is high and the promised resource has been sent, but not yet received and is thus conceptually owned by the channel. Lastly, in the Done state the flag is high and the promised resource has been sent and received. Each abstract state is associated with an invariant by the interpretation function $I_{\mathrm{b}}$, defined as follows:

$$
\begin{aligned}
& I_{\mathrm{b}}(x, P)(\text { Low }) \triangleq x \text {.flag } \mapsto 0 \\
& I_{\mathrm{b}}(x, P)(\text { High }) \triangleq x \text {.flag } \mapsto 1 * \text { stable }(P) * P \\
& I_{\mathrm{b}}(x, P) \text { (Done) } \triangleq x \text {.flag } \mapsto 1
\end{aligned}
$$

Here $x$ is the location of the channel, while $P$ is the resource controlled by the channel. Note that to move from the Low to the High abstract state the client must transfer ownership of $P$ to the shared region, in addition to setting the flag. Likewise, to move from the High to the Done abstract state the client may transfer $P$ out of the shared region and into its local state.

We want to ensure that only the sender (i.e., the owner of the send resource) is allowed to transition the abstract state from Low to High and only the receiver from High to Done. This will force the sender to transfer $P$ to the shared region upon setting the flag and allow the receiver to take ownership of $P$ once the flag has been set. In iCAP, we express this by labelling the Low to High transition with a set action and giving the sender exclusive ownership of the set action. The transition relation $T_{\mathrm{b}}$ thus has the following form. Each transition corresponds to an operation that can be performed on the channel. 


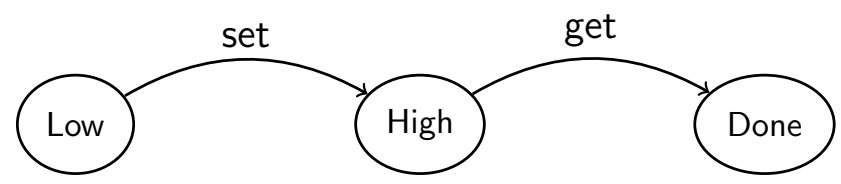

Using these definitions, we can give the predicates send and recv an interpretation:

$$
\begin{aligned}
\operatorname{send}(x, P) & \triangleq \exists r \cdot \operatorname{region}\left(\{\text { Low }\}, T_{\mathrm{b}}, I_{\mathrm{b}}(x, P), r\right) *[\text { set }]_{1}^{r} \\
\operatorname{recv}(x, P) & \triangleq \exists r \cdot \operatorname{region}\left(\{\text { Low, High }\}, T_{\mathrm{b}}, I_{\mathrm{b}}(x, P), r\right) *[\text { get }]_{1}^{r}
\end{aligned}
$$

The send predicate asserts that the abstract state is Low, since the promised resource has not been sent yet. On the other hand, recv asserts that the abstract state is either Low or High, but not Done, as the receiver does not know whether the promised resource has been sent yet, only that it has not been received yet. [set $]_{1}^{r}$ and $[\text { get }]_{1}^{r}$ are tokens allowing the thread to take particular transitions in $T_{\mathrm{b}}$. The send predicate allows the sender to set the flag and supply the promised resource, while recv allows the receiver to retrieve the promised resource.

Stability. Region assertions allow us to describe our knowledge about the current abstract state of a region. However, since regions are shared, concurrently executing threads may update the abstract state of regions, invalidating our region assertions in the process. An assertion is said to be stable if it is invariant under possible interference from the environment. Since the concrete send resource defined above asserts exclusive ownership of the set action, the environment cannot use this transition to update the abstract state. Hence, if we own the send resource, the environment cannot invalidate our knowledge that the current abstract state is Low; thus, the send resource is stable.

More generally, a region assertion, region $(R, T, I, r)$, is stable if $R$ is closed under all transitions in $T$ labelled with actions potentially owned by the environment. This is captured by the following iCAP proof rule.

$$
(\forall \alpha \notin A . \forall x \in X . T(\alpha)(x) \subseteq X) \Longrightarrow \operatorname{stable}\left(\operatorname{region}(X, T, I, r) * \circledast_{\alpha \in A}[\alpha]_{1}^{r}\right)
$$

Since the assertion asserts exclusive ownership of all the action in $A\left(\circledast_{\alpha \in A}[\alpha]_{1}^{r}\right)$, the environment cannot own any actions in $A$. Hence, the region assertion is stable if $X$ is closed under any transitions $\alpha$ not in $A$, as expressed by the assumption.

Stability is closed under several of the usual connectives of higher-order separation logic $\left(\perp, \top, \vee, \wedge, \forall, \exists,=_{\tau}\right.$, emp,$\left.*\right)$, but generally not under implication and separating implication. To avoid reasoning about stability of separating implication, we use Wickerson et al's explicit stabilisation operators, $\lfloor-\rfloor$ and $\lceil-\rceil$, instead of requiring $P$ to be stable [Wickerson et al. 2010]. We explicitly stabilise using $\lfloor P\rfloor$ and $\lceil P\rceil$ which are stable by construction for any assertion $P .\lfloor P\rfloor$ stands for the weakest assertion stronger than $P$ that is stable and $\lceil P\rceil$ for the strongest assertion weaker than $P$ that is stable. Thus, if $P$ is already stable, explicitly stabilising $P$ does nothing:

$$
\text { stable }(P) \Longrightarrow(\lfloor P\rfloor \Longleftrightarrow P \Longleftrightarrow\lceil P\rceil)
$$

However, in general, only the right implications hold: $\lfloor P\rfloor \Rightarrow P \Rightarrow\lceil P\rceil$. Explicit stabilisation operators are semi-distributive over separating conjunction:

$$
\lfloor P\rfloor *\lfloor Q\rfloor \Longrightarrow\lfloor P * Q\rfloor \quad\lceil P * Q\rceil \Longrightarrow\lceil P\rceil *\lceil Q\rceil
$$

As a result, they are easy to move around in proofs.

The concrete recv resource defined above is thus also easily shown to be stable, as \{Low, High\} is closed under the set transition, which is the only transition potentially owned by the environment. 
When reasoning about non-atomic statements, iCAP requires that the pre- and postcondition is stable, to account for possible interference from the environment. We use standard Hoare triples, written $\{P\} C\{Q\}$, when reasoning about non-atomic statements and angled triples, written $\langle P\rangle C\langle Q\rangle$, when reasoning about atomic statements. For every standard Hoare triple, $\{P\} C\{Q\}$, we implicitly have to prove stability of $P$ and $Q$. For the majority of the proof outlines in this article, these stability proofs are trivial and will be omitted. The following structural rule allows us to switch from atomic to non-atomic triples:

$$
\text { atomic }(C) \wedge \operatorname{stable}(P) \wedge \operatorname{stable}(Q) \wedge\langle P\rangle C\langle Q\rangle \Longrightarrow\{P\} C\{Q\}
$$

The predicate atomic holds for any command which is assumed to be atomic by iCAP: these are CAS, field read, field assignment and stack assignment.

Higher-order shared resources. The idea of shared resources that must satisfy an invariant or evolve following a protocol could equally be expressed in prior logics such as CAP or RGSep [Vafeiadis and Parkinson 2007; Dinsdale-Young et al. 2010]. The distinction with iCAP is that it is based on a higher-order separation logic and supports shared higher-order resources - i.e., shared regions containing shared resources. For instance, the send resource defined above is parametric in the promised resource $P$, which the client is free to instantiate with a shared resource - for example, another synchronisation construct.

It is well-known that reasoning about shared higher-order resources is difficult (for example, see the problems with our previous paper, discussed in §8). Intuitively, this is because the semantics of protocols is defined in terms of the semantics of assertions, but assertions are defined in terms of protocols. To avoid this problematic circularity, iCAP stratifies the construction of the semantic domain of protocols using step-indexing. As a result of this stratification, assertions and specifications are only given meaning with respect to a step-index.

In the case of self-referential protocols, step-indexing breaks the circularities by interpreting a region assertion at step-index $n+1$ in terms of the semantics of the region interpretation at step-index $n$. For instance, a region assertion region $(\{x\}, T, I, r)$ holds at step-index $n+1$, if the shared resources owned by region $r$ satisfies $I(x)$ at step-index $n$. To capture this syntactically, iCAP introduces a 'later' modality, written $\triangleright$. The assertion $\triangleright P$ holds at step-index $n+1$, if $P$ holds at step-index $n$. The region assertion region $(\{x\}, T, I, r)$ thus expresses that the shared region $r$ currently owns the resources described by $\triangleright I(x)$.

The semantics of Hoare triples connects the step-indexes of the pre- and postcondition with execution steps. Disregarding environment interference, a specification $\{P\} C\{Q\}$ is valid, if for any number of steps $n$ and any initial state in $P$ at step-index $n$, if $C$ terminates in $k \leq n$ steps, then the terminal states satisfies $Q$ at step-index $n-k$. The effects of this stratification and connection of step-indices with operational steps, is that reasoning involving shared resources require a corresponding step in the program execution. Intuitively, the logic must 'wait a step' before examining resources transferred out of shared regions, to prevent cycles in the reasoning. Formally, this is expressed by the frame rule for atomic commands (AFrame) given below, which allows the removal of a later from the frame. In particular, if $\triangleright P$ holds for the frame at step-index $n$ then $P$ holds for the frame at step-index $n-1$. Thus, since the atomic command requires exactly one step of execution, $P$ holds in the post-condition, as the post-condition is evaluated at step-index $n-1$.

$$
\text { stable }(R) \wedge\langle P\rangle C\langle Q\rangle \Longrightarrow\langle P * \triangleright R\rangle C\langle Q * R\rangle
$$

One intuition for the step-indexed model is that an assertion $P$ holds at step-index $n$ if the assertion that it makes about the state cannot be invalidated with $n$ or less steps of execution. Consequently, if an assertion $P$ holds at step-index $n+1$ it also holds at stepindex $n$, since any assertion that can be invalidated in $n$ or less steps, can also be invalidated in $n+1$ or less steps. This property is expressed by the (SMONo) rule given below, which 
expresses that if $P$ holds now, it also holds with one less step of execution left $(\triangleright P)$.

$$
P \Longrightarrow \triangleright P
$$

Using (SMono) and the rule of consequence, one can derive the standard CAP frame rule. The assertion $\triangleright P$ is stable if $P$ is stable.

The effect of introducing $\triangleright$ is that accesses to shared resources in regions coincide with operational steps in the program. This breaks the circularity and gives iCAP a well-defined semantics.

However, it also means that splitting and renunciation must be associated with an explicit skip instruction to justify the transfer of shared resources in and out of regions. While these skip instructions are crucial to the well-definedness of protocols, they can typically be eliminated once we consider a whole program. In particular, for pre- and postconditions expressible in first-order separation logic, iCAP is adequate with respect to first-order separation logic [Svendsen and Birkedal 2014b, Theorem 1] and in first-order separation logic skip instructions can freely be eliminated. Hence, if $P$ and $Q$ are expressible in first-order separation logic and $\vdash_{i C A P}\{P\} C\{Q\}$, then $\vdash_{S L}\{P\} \tilde{C}\{Q\}$, where $\tilde{C}$ is $C$ stripped of skip instructions.

The later operator distributes over conjunction, disjunction, separating conjunction, stabilization brackets. It semi-distributes over implication and separating implication, i.e:

$$
\triangleright(P \Rightarrow Q) \Longrightarrow(\triangleright P \Rightarrow \triangleright Q) \quad \triangleright(P * Q) \Longrightarrow(\triangleright P * \triangleright Q)
$$

Later also distributes over existential and universal quantification over non-empty types. See Appendix C.2.2 for proof rules. In proof outlines we sometimes apply these properties silently.

Reasoning about shared regions. All statements that access resources owned by shared regions must be atomic and obey the protocols of the regions involved. This is achieved using structural rules that allow shared resources to be treated as local resources for the duration of an atomic statement. We refer to these rules as "region opening" rules and as "entering" and "exiting" a shared region, when applying these rules in proof outlines.

Conceptually, these rules require us to prove 1) that we own sufficient action permissions to justify any potential updates of the abstract state and 2) that we transfer the appropriate resources back to the shared region after the atomic statement. To illustrate, consider verifying the signal method of the simplified barrier implementation:

$$
\{\operatorname{send}(\mathrm{i}, P) * \operatorname{stable}(P) * P\} \text { i->flag }:=1\{\mathrm{emp}\}
$$

Since raising the flag is an atomic statement and both the pre- and postcondition is stable, we can switch to atomic triples. This frees us from reasoning about stability and possible interference during the execution of the atomic statement. After unfolding the send resource we are thus left with the following proof obligation:

$$
\left\langle\operatorname{region}\left(\{\operatorname{Low}\}, T_{\mathrm{b}}, I_{\mathrm{b}}(\mathrm{x}, P), r\right) *[\mathrm{set}]_{1}^{r} * \operatorname{stable}(P) * P\right\rangle \mathrm{x}->\mathrm{fl} \text { lag }:=1\langle\mathrm{emp}\rangle
$$

The precondition asserts that the flag field is owned by the shared region $r$. To update the field we are thus forced to open the shared region governing the given channel and thus to respect its protocol. Intuitively, upon raising the flag the abstract state changes from Low to High. We can thus strengthen the proof obligation:

$$
\begin{gathered}
\left\langle\operatorname{region}\left(\{\text { Low }\}, T_{\mathrm{b}}, I_{\mathrm{b}}(\mathrm{x}, P), r\right) *[\text { set }]_{1}^{r} * \operatorname{stable}(P) * P\right\rangle \\
\mathrm{x}->\text { flag }:=1 \\
\left\langle\operatorname{region}\left(\{\text { High }\}, T_{\mathrm{b}}, I_{\mathrm{b}}(\mathrm{x}, P), r\right)\right\rangle
\end{gathered}
$$

Note that this postcondition is not stable under get transitions. However, since we are reasoning about an atomic statement, the postcondition is not required to be stable. 
To discharge the above proof obligation we first have to prove that we are allowed to update the abstract state from Low to High. Since the precondition asserts exclusive ownership of the set transition, this reduces to proving that there exists a set-labelled path from Low to High in $T_{\mathrm{b}}$, which is true by definition. Secondly, we must prove that $\mathrm{x}->\mathrm{flag}:=1$ does indeed transform the resources associated with abstract state Low to those associated with abstract state High, according to $I_{\mathrm{b}}(\mathrm{x}, P)$. We are thus left with the following proof obligation:

$$
\left\langle\triangleright I_{\mathrm{b}}(\mathrm{x}, P)(\text { Low }) *[\mathrm{set}]_{1}^{r} * \operatorname{stable}(P) * P\right\rangle \mathrm{x}->\mathrm{flag}:=1\left\langle\triangleright I_{\mathrm{b}}(\mathrm{x}, P)(\text { High })\right\rangle
$$

Given local ownership of the shared resources for the abstract state Low we must transfer back resources corresponding to abstract state High after the execution of the atomic statement. Since protocols are implicitly interpreted one step later, the shared resources are only available one step later in the precondition and only have to be provided one step later in the postcondition. After unfolding $I_{\mathrm{b}}(x, P)$ and applying SMONO we are left with the following proof obligation:

$$
\left\langle(\triangleright \mathrm{x} \text {.flag } \mapsto 0) *[\mathrm{set}]_{1}^{r} * \operatorname{stable}(P) * P\right\rangle \mathrm{x}->\text { flag }:=1\langle\mathrm{x} \text {.flag } \mapsto 1 * \operatorname{stable}(P) * P\rangle
$$

Conceptually, this is provable because step-indexing only affects assertions that can generate problematic circularities in the domain. Primitive points-to assertions such as $x . f \mapsto v$ only affect addresses and values, and are thus independent of the step-indexing. This is captured by the structural (LPOINTS) rule given below, which allows us to remove a $\triangleright$ from the pre-condition of a points-to assertion.

$$
\langle x . f \mapsto y\rangle C\langle Q\rangle \Longrightarrow\langle\triangleright x . f \mapsto y\rangle C\langle Q\rangle
$$

The general iCAP proof rule for accessing shared regions is given below:

$$
\begin{gathered}
\forall x \in X .(x, f(x)) \in \overline{T(A)} \\
\frac{\forall x \in X .\left\langle P * \circledast \alpha \in A[\alpha]_{g(\alpha)}^{r} * \triangleright I(x)\right\rangle C\langle Q(x) * \triangleright I(f(x))\rangle^{\mathcal{E}}}{\left\langle P * \circledast_{\alpha \in A}[\alpha]_{g(\alpha)}^{r} * \operatorname{region}(X, T, I, r)\right\rangle C\langle\exists x . Q(x) * \operatorname{region}(\{f(x)\}, T, I, r)\rangle^{\mathcal{E} \uplus\{r\}}} \text { (AOPEN) }
\end{gathered}
$$

It generalizes the above example by considering a set of possible initial abstract states $X$ and allowing the client to transfer ownership of resources in and out of the shared region using $P$ and $Q$. To apply the rule we must define a function $f$ that for every possible initial abstract state $x \in X$ defines the desired terminal abstract state $f(x)$. The first premise asserts that for every possible initial abstract state $x$ there exists a path from $x$ to $f(x)$ labelled with actions from the set $A$ (we use $\bar{R}$ as notation for the reflexive, transitive closure of the relation $R$ ). Since the precondition asserts non-exclusive ownership of every action in $A$, this ensures that we are allowed to update the abstract state from $x$ to $f(x)$ for every possible initial state $x \in X$. The $g$ function, which records the fractional ownership of each action $\alpha \in A$, ensures that we own the same action fractions in the assumption and conclusion of the rule. We implicitly require that $g(\alpha) \neq 0$ for all $\alpha \in A$. The second premise ensures that $C$ does indeed transform the resources associated with the abstract state $x$ to $f(x)$ for every possible initial abstract state $x \in X$.

Since opening a shared region grants local ownership of the region's current resources, in general it would be unsound to have two nested openings of the same region in the proof tree, as this would duplicate the region's resources. To avoid this, we annotate the postcondition of atomic triples with a region mask, $\mathcal{E}$, of regions that may be opened. To open the region $r$, the above proof rule requires that $r$ is in the region mask and removes it from the region mask in the premise, to ensure $r$ is not opened again.

Verifying signal and wait. Figures 10 and 11 sketch proofs for signal() and wait() respectively. The proof of wait (Fig. 11) uses similar reasoning to signal. The main difference is that the region has two initial abstract states, Low and High, and that the thread 
holds the token get, allowing it to transition from High to Done. We deal with the Low and High cases separately - see bottom left and right of Fig. 11. In the Low case, the resource has not been sent yet and we close the region in the Low state again. In the High case, the resource has been sent and we use the thread's get token to take ownership of $P$ and close the region in the Done state.

iCAP is an intuitionistic separation logic, meaning it admits weakening, and in particular that $P * Q \Longrightarrow Q$. We often use this property to dispose of unwanted predicates - intuitively, we can forget they exist. For example, we delete a redundant set token on line 11 of Figure 10.

View-shifts. The region opening rule given above allows a region to be opened for the duration of an atomic statement. It is also possible to open a shared region and close it immediately before the next statement is executed. This is for instance useful to abstractly describe transfer of resources in and out of shared regions. iCAP expresses such updates of the abstract state that do not affect the concrete state using the view-shift operator, $\sqsubseteq$.

For instance, the following view-shift expresses that if the channel is in the abstract state High and we own the get action, then we can change the abstract state to Done and take ownership of $\triangleright P$.

$$
\text { region }\left(\{\mathrm{High}\}, T_{\mathrm{b}}, I_{\mathrm{b}}(x, P), r\right) *[\text { get }]_{1}^{r} \sqsubseteq \operatorname{region}\left(\{\text { Done }\}, T_{\mathrm{b}}, I_{\mathrm{b}}(x, P), r\right) *[\text { get }]_{1}^{r} * \triangleright P
$$

View-shifts generalize standard implication and satisfy a generalized rule of consequence:

$$
P_{1} \sqsubseteq P_{2} \wedge\left\{P_{2}\right\} C\left\{Q_{2}\right\} \wedge Q_{2} \sqsubseteq Q_{1} \Longrightarrow\left\{P_{1}\right\} C\left\{Q_{1}\right\}
$$

We can thus use view-shifts to factor manipulations of shared resources that do not affect the concrete state. The view-shift region opening rule is very similar to the region opening rule for atomic statements and also requires proving that any updates of the abstract state are permitted and that resources are transformed correctly:

$$
\frac{\left.\forall x \in X .(x, f(x)) \in \overline{T(A)} \quad \forall x \in X . P * \circledast_{\alpha \in A}[\alpha]_{g(\alpha)}^{r} * \triangleright I(x)\right\rangle \sqsubseteq^{\mathcal{E}} Q * \triangleright I(f(x))}{\left.P * \circledast_{\alpha \in A}[\alpha]_{g(\alpha)}^{r} * \text { region }(X, T, I, r)\right\rangle \sqsubseteq^{\mathcal{E} \uplus\{r\}} Q * \operatorname{region}(\{f(x)\}, T, I, r)} \text { (VOpen) }
$$

Just as for atomic commands, we need to ensure that already opened regions cannot be opened again. We thus index view-shifts with a region mask annotation $(\mathcal{E})$ that describes the set of regions that may be opened.

Allocation of shared regions is also described using view shifts. To allocate a shared region in initial abstract state $x$ with interpretation map $I$ we must transfer the resource $I(x)$ to the shared region. This is expressed by the following view-shift axiom:

$$
(\forall x \text {. stable }(I(x))) \Longrightarrow I(x) \sqsubseteq \exists r \text {. region }(\{x\}, T, I, r) * \circledast \circledast_{\alpha \in A}[\alpha]_{1}^{r}
$$

The resources owned by a shared region must always be stable. This is enforced upon allocation of the region, as expressed by the above axiom. Upon allocating a shared region, we can take exclusive ownership of any set of actions $A$ on $r$.

While iCAP supports recursively-defined higher-order shared resources, soundness of iCAP depends crucially on the fact that the transition systems associated with each region are not recursively-defined. This is enforced when allocating a new region. Formally, the VALLOC rule includes a side-condition on the labelled transition system, $T$, which is given as relation on abstract states indexed by an action identifier, to enforce this. This side-condition is trivially satisfied by every labelled transition system expressible in firstorder logic with equality. Since this is the case for all the transition systems employed in this article, we will not go into details about this technical side-condition. We refer the reader to the iCAP article for details [Svendsen and Birkedal 2014a].

Verifying newchan. Figure 9 shows a sketch proof of newchan. First the concrete channel data structure is allocated. This allows ownership of the flag field to be transferred to a newly allocated channel region, using the VALLOC and ACONSQ rule. 


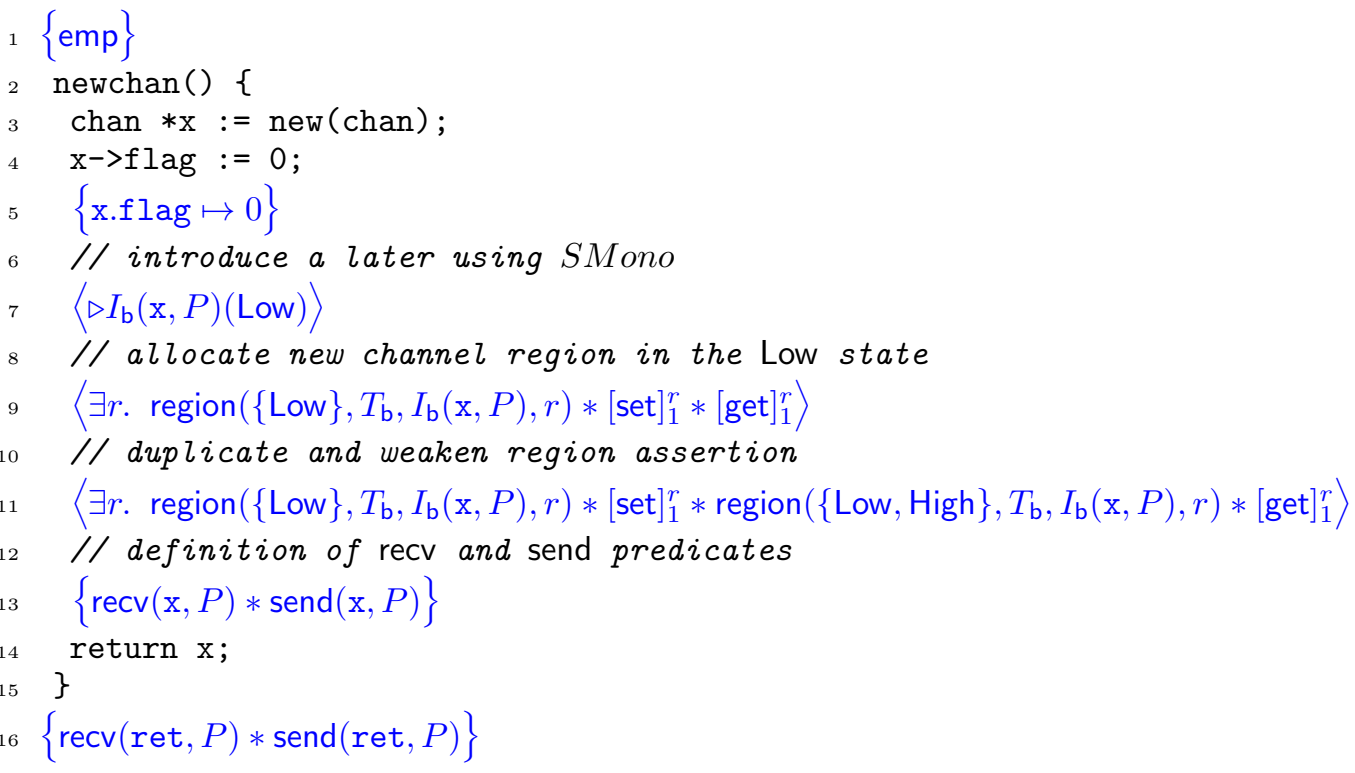

Fig. 9. Proof of newchan() using simple predicate definitions.

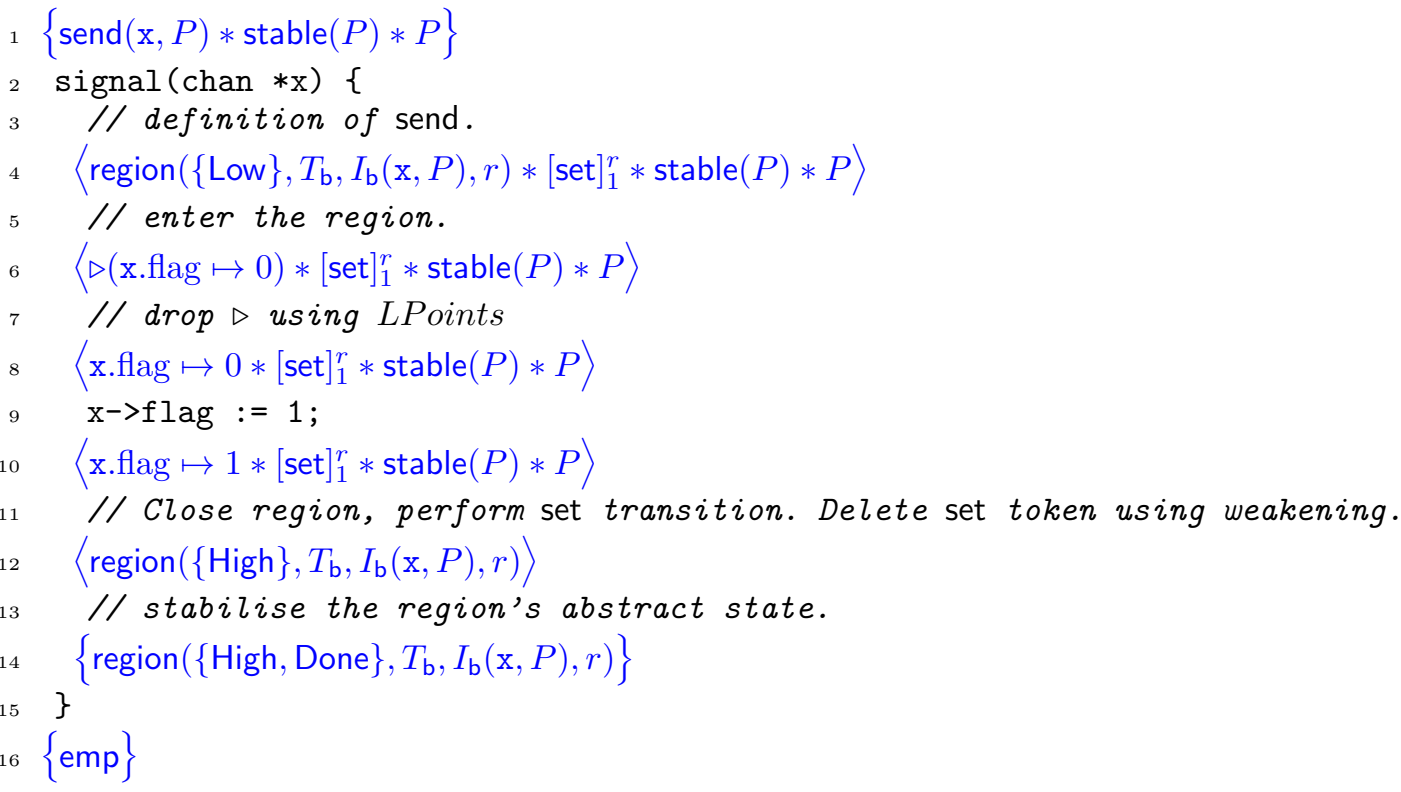

Fig. 10. Proof of signal() using simple predicate definitions. 


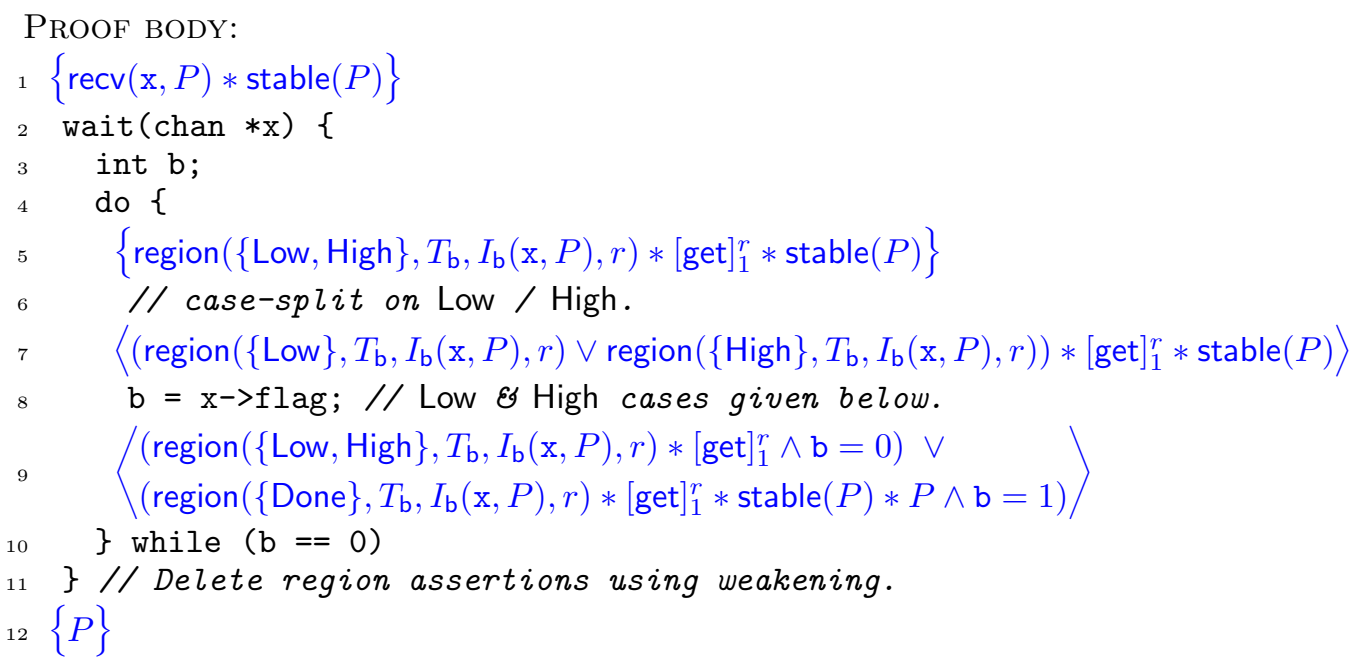

\section{LOW CASE:}

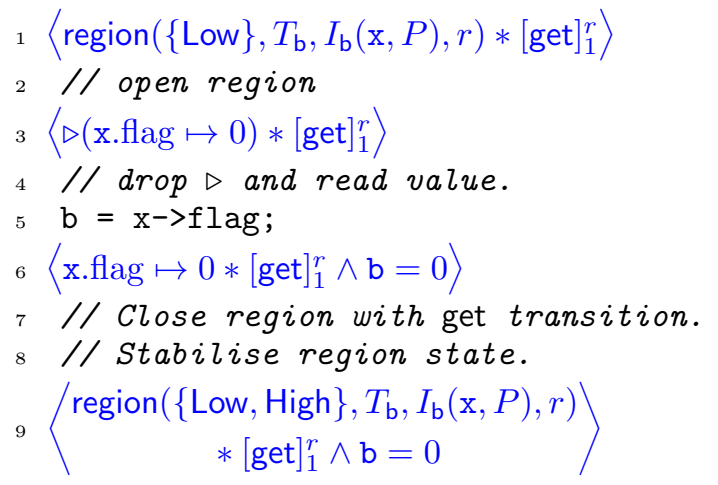

HigH CASE:

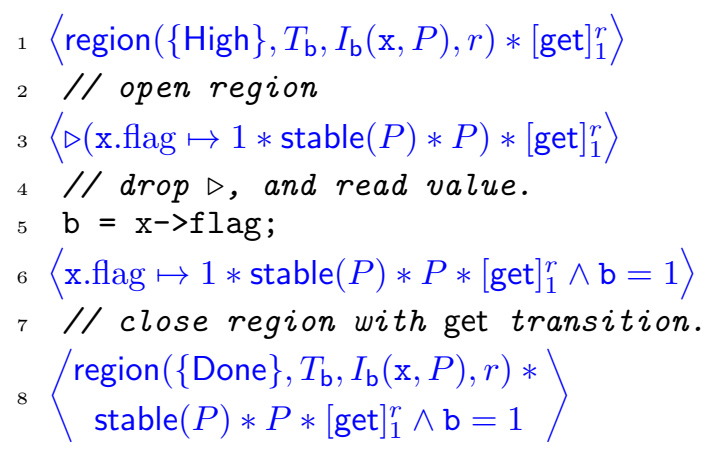

Fig. 11. Proof of wait() using simple predicate definitions.

\section{SPLITTING CHANNELS}

In this section, we extend our proof of the simple implementation to cover splitting - intuitively, promised resources can be divided between threads before they are sent. As the simple implementation sequentialises signalling, we leave extension and renunciation to $\S 6$.

Strengthened abstract specification. To manage promised split resources inside the proof, we use separating implication, * However, the stability assertion stable is difficult to reason about because it is does not distribute with respect to $*$. Explicit stabilization operators, $\lceil-\rceil$ and $\lfloor-\rfloor$ are easier to reason about, and we therefore define a channel specification written in terms of explicit stabilization, from which our client-facing abstract specification can be derived. This strengthened specification is given in Figure 12 .

To derive the weaker specification given in $\S 3.4$ from this stronger one, we define $\operatorname{send}_{w}(x, P)$ in the weaker specification as $\exists P^{\prime}$. $\operatorname{send}_{s}\left(x, P^{\prime}\right) \wedge \operatorname{valid}\left(P \Rightarrow P^{\prime}\right)$, using the send $d_{s}$ predicate from the stronger specification. The valid predicate allows us to utilise facts about iCAP axioms in our library definitions and specifications. For further discussion, see Appendix C.2.1. Predicates other than send are lifted to the new specification without modification.

Function specifications then follow directly from stable $(P) \Longrightarrow(\lfloor P\rfloor \Leftrightarrow P \Leftrightarrow\lceil P\rceil)$. 


$$
\begin{aligned}
& \text { Specifications: } \quad\{\operatorname{emp}\} \quad i=\operatorname{newchan}() \quad\{\operatorname{recv}(i, P) * \operatorname{send}(i, P)\} \\
& \{\operatorname{send}(i, P) *\lfloor P\rfloor\} \quad \text { signal (i) } \quad\{\text { emp }\} \\
& \{\operatorname{recv}(\mathrm{i}, P)\} \quad \text { wait }(\mathrm{i}) \quad\{\lceil P\rceil\} \\
& \left\{\begin{array}{l}
\operatorname{send}(\mathrm{x}, P) * \\
\circledast_{e \in E} e \prec \mathrm{x} * \circledast_{l \in L} \mathrm{x} \prec l
\end{array}\right\} \quad(\mathrm{b}, \mathrm{a})=\operatorname{extend}(\mathrm{x})\left\{\begin{array}{l}
\operatorname{send}(\mathrm{b}, Q) * \operatorname{recv}(\mathrm{b}, Q) * \operatorname{send}(\mathrm{a}, P) \\
* \mathrm{~b} \prec \mathrm{a} * \circledast_{e \in E} e \prec \mathrm{b} * \circledast_{l \in L} \mathrm{a} \prec l
\end{array}\right\}
\end{aligned}
$$

Axioms: $\quad x \prec y \quad \Longrightarrow \quad x \prec y * x \prec y$

$$
\begin{array}{rcl}
x \prec y * y \prec z & \Longrightarrow & x \prec z \\
\{\operatorname{recv}(x, P) * \operatorname{send}(y, Q) * x \prec y\} & \langle\operatorname{skip}\rangle & \{\operatorname{send}(y, P * Q)\} \\
\left\{\operatorname{recv}(a, P) *\left\lfloor\lceil P\rceil *\left(P_{1} * P_{2}\right)\right\rfloor\right\} & \langle\operatorname{skip}\rangle & \left\{\operatorname{recv}\left(a, P_{1}\right) * \operatorname{recv}\left(a, P_{2}\right)\right\}
\end{array}
$$

Fig. 12. Full specification with explicit stabilisation.

To derive the weakened renunciation axiom, we reason as follows:

$$
\begin{aligned}
&\left\{\operatorname{send}_{w}(x, P * Q) * \operatorname{recv}(y, P) * x \prec y\right\} \\
& \Longleftrightarrow\left\{\exists P^{\prime} \cdot \operatorname{send}_{s}\left(x, P^{\prime}\right) * \operatorname{valid}\left((P * Q) \Rightarrow P^{\prime}\right) * \operatorname{recv}(y, P) * x \prec y\right\} \\
& \Longleftrightarrow\left\{\exists P^{\prime} \cdot \operatorname{send}_{s}\left(x, P^{\prime}\right) * \operatorname{valid}\left(Q \Rightarrow\left(P * P^{\prime}\right)\right) * \operatorname{recv}(y, P) * x \prec y\right\} \\
&\langle\operatorname{skip}\rangle \\
&\left\{\exists P^{\prime} \cdot \operatorname{send}_{s}\left(x, P * P^{\prime}\right) * \operatorname{valid}\left(Q \Rightarrow\left(P * P^{\prime}\right)\right)\right\} \\
& \Longleftrightarrow\left\{\operatorname{send}_{w}(Q)\right\}
\end{aligned}
$$

In our strengthened specification, splitting is expressed by the following axiom:

$$
\left\{\operatorname{recv}(a, P) *\left\lfloor\lceil P\rceil *\left(P_{1} * P_{2}\right)\right\rfloor\right\} \quad\langle\operatorname{skip}\rangle \quad\left\{\operatorname{recv}\left(a, P_{1}\right) * \operatorname{recv}\left(a, P_{2}\right)\right\}
$$

To derive the weaker splitting axiom note that if stable $(P * Q)$ then:

$$
\top \Rightarrow\lfloor\top\rfloor \Rightarrow\lfloor P * Q * P * Q\rfloor \Rightarrow\lfloor\lceil P * Q\rceil * P * Q\rfloor
$$

In the remainder of the paper, we verify our implementations against the more general specification given in Figure 12.

Abstract state. To begin the proof, we first define the set of abstract states. In the absence of splitting, the entire promised resource is transferred to a single recipient. For each channel, the resource can therefore be either unsent, sent but not received, or received. In Section 4 we thus introduced three abstract states (Low, High and Done) to represent these three situations.

Splitting means that promised resources can be logically divided between recipients. The abstract state must therefore also track how the promised resource has been split and (if it has been sent) which recipients have taken ownership. Intuitively, we parameterise the abstract state with sets of propositions describing the splitting. We would then have abstract states $\operatorname{Low}(\mathcal{I})$ and $\operatorname{High}(\mathcal{I})$, with $\mathcal{I} \in \mathcal{P}_{\text {fin }}(\operatorname{Prop})$. Each $P \in \mathcal{I}$ represents a promise to a recipient. In the abstract state $\operatorname{Low}(\mathcal{I})$, the whole resource, which has yet to be sent, has been split into a set of promised resources $\mathcal{I}$. In the abstract state $\operatorname{High}(\mathcal{I})$, the entire promised resource has been sent, and the portions still in $\mathcal{I}$ have yet to be received.

In the presence of splitting, $\operatorname{recv}(a, P)$ only confers the right to receive and take ownership of the portion of the resource represented by $P$. We capture this by indexing the split and 
receive transition with a proposition describing the associated resource. We thus have two transitions: send for sending the entire promised resource and change ${ }_{P}$ for splitting the resource $P$ or taking ownership of $P$.

$$
\begin{aligned}
\text { send : } \operatorname{Low}(\mathcal{I}) & \leadsto \operatorname{High}(\mathcal{I}) \\
\text { change }_{P}: \operatorname{Low}(\mathcal{I} \uplus\{P\}) & \leadsto \operatorname{Low}\left(\mathcal{I} \uplus\left\{P_{1}, P_{2}\right\}\right) \\
\text { change }_{P}: \operatorname{High}(\mathcal{I} \uplus\{P\}) & \leadsto \operatorname{High}\left(\mathcal{I} \uplus\left\{P_{1}, P_{2}\right\}\right) \\
\text { change }_{P}: \operatorname{High}(\mathcal{I} \uplus\{P\}) & \leadsto \operatorname{High}(\mathcal{I})
\end{aligned}
$$

Note that when splitting $P$ using the change ${ }_{P}$ transition, the transition system does not enforce that $P * P_{1} * P_{2}$. Rather, this will be enforced by the interpretation function for the abstract states.

The transition system described in this section unfortunately cannot be expressed directly in iCAP. This is because iCAP's abstract states and transitions cannot be directly indexed by propositions. It is unclear how this restriction could be lifted in iCAP's step-indexing framework.

Instead, iCAP supports saved propositions, an encoding which allows propositions to be associated with identifiers and stored. To formalize the above transition system, we index states and transitions with these identifiers. This skirts the restrictions imposed by step-indexing, and allows the reasoning we want. In the following section we introduce the necessary logical machinery.

\subsection{Saved Propositions}

A saved proposition, written $r \stackrel{\pi}{\Leftrightarrow} P$, associates an identifier $r$ with a proposition $P$. By introducing the indirection from identifiers to propositions we lose some properties. Most importantly, we cannot easily unify saved propositions: given $r \stackrel{\pi_{1}}{\Longleftrightarrow} P$ and $r \stackrel{\pi_{2}}{\Longleftrightarrow} Q$, in general it does not hold that $P=Q$. However, saved propositions still satisfy enough properties that we can verify the splitting axiom.

In addition to the identifier $r$ and proposition $P$, we also have a fractional parameter $\pi \in(0,1]$ which records how the saved proposition has been shared between threads. In other words, it serves the same role as fractional permissions for heap cells in standard separation logic [Bornat et al. 2005].

We require that saved propositions satisfy the following three properties:

$$
\begin{aligned}
\text { emp } & \Longrightarrow \exists r . r \stackrel{1}{\models} P \\
r \stackrel{\pi_{1}}{\Longrightarrow} P * r \stackrel{\pi_{2}}{\Longrightarrow} P & \Longleftrightarrow \begin{cases}r \stackrel{\pi_{1}+\pi_{2}}{\Longrightarrow} P & \text { if }\left(\pi_{1}+\pi_{2}\right) \leq 1 \\
\text { false } & \text { otherwise }\end{cases} \\
r \stackrel{\pi_{1}}{\Longrightarrow} P * r \stackrel{\pi_{2}}{\Longrightarrow} Q & \Longrightarrow r \stackrel{\pi_{1}}{\Longrightarrow} P * r \stackrel{\pi_{2}}{\Longrightarrow} Q *(\triangleright P \Rightarrow \triangleright Q)
\end{aligned}
$$

Property (1) allows us to create a saved proposition for an arbitrary proposition P. Property (2) says that saved propositions are linear, meaning we can split and join them without worrying about unwanted duplication. Observe that the fractions $\pi_{1}$ and $\pi_{2}$ are used to track splitting. Property (3) says that holding two saved propositions on the same region allow us to convert from one to another. This is a corrected version of the unification property discussed above. The iCAP later operator ' $\triangleright$ ' is needed because we use shared regions internally in the definition of saved propositions.

The following additional property is derivable from property (3) - proved in Lemma A.1. 


$$
\left(\begin{array}{c}
r \stackrel{\pi_{1}}{\Longleftrightarrow} P * r \stackrel{\pi_{2}}{\Longleftrightarrow} Q * \\
(X * \triangleright(Q * Y)) *(P * Z)
\end{array}\right) \quad \Longrightarrow \quad r \stackrel{\pi_{1}}{\Longleftrightarrow} P * r \stackrel{\pi_{2}}{\Longleftrightarrow} Q *(X * \triangleright(Z * Y))
$$

This says that we can apply property (3) inside separating implications. This is useful when modifying a resource embedded into a larger assertion.

We can encode and verify saved propositions as predicates in iCAP - they do not require any extension of the logic. Our encoding is given in Appendix A. In our encoding, region identifiers are used as identifiers for saved propositions - this is because internally saved propositions are encoded by regions.

\subsection{Predicate Definitions for send and recv}

Once again, we begin by defining the structure of a region. Abstract states are now terms of the form $\operatorname{Low}(\mathcal{I})$ and $\operatorname{High}(\mathcal{I})$, where $\mathcal{I}$ is a finite set of identifiers in $\mathcal{P}_{\text {fin }}(\mathrm{RId})$. We use LoHi to stand for either Low or High. The $\mathcal{I}$ parameter represents the set of outstanding obligations, i.e. the resources that other threads expect to be supplied. As described above, we use saved propositions to give an interpretation to these sets of region identifiers. If we have the abstract state $\operatorname{Low}(\mathcal{I})$ or $\operatorname{High}(\mathcal{I})$, then each $i \in \mathcal{I}$ corresponds to a resource promised to some thread. To find out what resource $P$ is expected, we examine the associated saved proposition $i \stackrel{\pi}{\Rightarrow} P$.

Actions in the transition relation $T_{\mathrm{m}}$ are of the form send and change $(i)$, where $i$ is the identifier for a saved proposition. The first action, send, sets the flag, and simply moves from Low to High. The second, change $(i)$, is both the action of taking the resource associated to region $i$ when the flag is high, and splitting the resource associated with identifier $i$ to the resource required by $i_{1}$ and $i_{2}$. These new identifiers $i_{1} / i_{2}$ can be chosen arbitrarily: however, the invariant mapping $I_{\mathrm{m}}$ ensures they are properly associated with saved propositions.

$$
\begin{aligned}
T_{\mathrm{m}}(\text { send }) & \triangleq\{(\operatorname{Low}(\mathcal{I}), \operatorname{High}(\mathcal{I}))\} \\
T_{\mathrm{m}}(\operatorname{change}(i)) \triangleq\{(\operatorname{High}(\mathcal{I} \uplus\{i\}), \operatorname{High}(\mathcal{I}))\} & \cup\left\{\left(\operatorname{Low}(\mathcal{I} \uplus\{i\}), \operatorname{Low}\left(\mathcal{I} \uplus\left\{i_{1}, i_{2}\right\}\right)\right)\right\} \\
& \cup\left\{\left(\operatorname{High}(\mathcal{I} \uplus\{i\}), \operatorname{High}\left(\mathcal{I} \uplus\left\{i_{1}, i_{2}\right\}\right)\right)\right\}
\end{aligned}
$$

The invariants associated with Low and High are defined as follows:

$$
\begin{aligned}
& I_{\mathrm{m}}(x, r, P)(\operatorname{Low}(\mathcal{I})) \triangleq x \text {.flag } \mapsto 0 * \text { waiting }(P, \mathcal{I}) * \text { changes }_{r}(\mathcal{I}) \\
& I_{\mathrm{m}}(x, r, P)(\operatorname{High}(\mathcal{I})) \triangleq x \text {.flag } \mapsto 1 * \operatorname{ress}(\mathcal{I}) * \operatorname{changes}_{r}(\mathcal{I}) \\
& \text { where } \\
& \text { waiting }(P, \mathcal{I}) \triangleq \exists Q: \mathcal{I} \rightarrow \text { Prop. }\left\lfloor(\triangleright P) * \circledast_{i \in \mathcal{I}}^{\circledast} \cdot Q(i)\right\rfloor * \underset{i \in \mathcal{I}}{\circledast} \cdot i \stackrel{1 / 2}{\Longrightarrow} Q(i) \\
& \operatorname{ress}(\mathcal{I}) \triangleq \underset{i \in \mathcal{I}}{\circledast} \exists R .(i \stackrel{1 / 2}{\Longleftrightarrow} R) *\lceil\triangleright R\rceil \\
& \operatorname{changes}_{r}(\mathcal{I}) \triangleq \underset{i \notin \mathcal{I}}{\circledast} \text {. }[\text { change }(i)]_{1}^{r}
\end{aligned}
$$

The definitions here use three auxiliary predicates: waiting, standing for resources that have been promised but not supplied; ress, standing for resources once they have been supplied; and changes, standing for change tokens for unused identifiers. The set changes can be seen as a 'library' of tokens which are not currently in use, and are currently not used by any thread. Having this library of tokens allows new saved propositions to be added when splitting. 


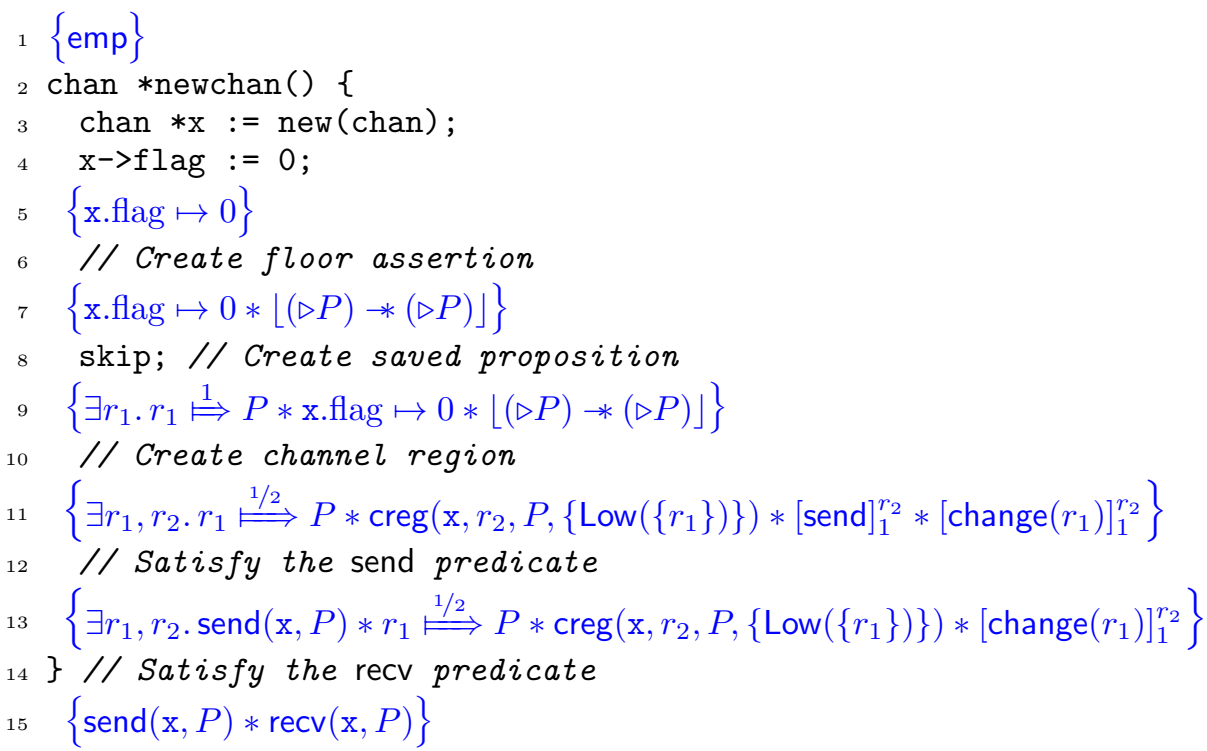

Fig. 13. Proof of newchan() w.r.t. the full specification.

The representation of Low consists of the flag, change tokens, and the waiting predicate. waiting $(P, \mathcal{I})$ requires the existence of a mapping $Q$ from region identifiers, to propositions representing obligations to other threads. The obligations for different threads are tied together using fractional saved propositions $i \stackrel{1 / 2}{\Longrightarrow} Q(i)$. The assertion $\left\lfloor(\triangleright P) * \circledast_{i \in \mathcal{I}} \cdot Q(i)\right\rfloor$ means that supplying the resource $P$ will satisfy each obligation $Q(i)$.

The representation of the High state consists of the flag and change tokens, and the ress predicate. $\operatorname{ress}(\mathcal{I})$ pairs together fractional saved propositions, $i \stackrel{1 / 2}{\longmapsto} R$ with resources $\lceil\triangleright R\rceil$. The other half of each saved proposition is held by the thread that has been promised the resource through the recv predicate (see below). This ensures that all threads that have been promised resources can claim them.

We use a shorthand for the region assertion in our definitions and proofs:

$$
\operatorname{creg}(x, r, P, S) \triangleq \operatorname{region}\left(S, T_{\mathrm{m}}, I_{\mathrm{m}}(x, r, P), r\right)
$$

The definition of the send predicate is now straightforward. It asserts that the region is in a Low state, and holds the unique permission to perform the send action.

$$
\operatorname{send}(x, P) \triangleq \exists r \cdot \operatorname{creg}(x, r, P,\{\operatorname{Low}(\mathcal{I}) \mid \operatorname{true}\}) *[\operatorname{send}]_{1}^{r}
$$

The definition of $\operatorname{recv}(x, Q)$ predicate is more complex. It includes $r^{\prime} \stackrel{1 / 2}{\Longleftrightarrow} Q$, half the permission on the saved proposition $Q$. It also asserts that $r^{\prime}$ is one of identifiers recorded in the region. This ensures that the resource retrieved from the shared region is the correct one, i.e. the one that was promised (see the next section for the reasoning steps involved).

$$
\operatorname{recv}(x, Q) \triangleq \exists R, r, r^{\prime} . \operatorname{creg}\left(x, r, R,\left\{\operatorname{LoHi}(\mathcal{I}) \mid r^{\prime} \in \mathcal{I}\right\}\right) * r^{\prime} \stackrel{1 / 2}{\Longleftrightarrow} Q *\left[\operatorname{change}\left(r^{\prime}\right)\right]_{1}^{r}
$$

\subsection{Proofs of newchan(), signal(), wait(), and the Splitting Axiom}

Proving newchan. The proof of newchan (Fig. 13) allocates a region containing the concrete channel state. Most steps in the proof are straightforward. The challenging ones are 


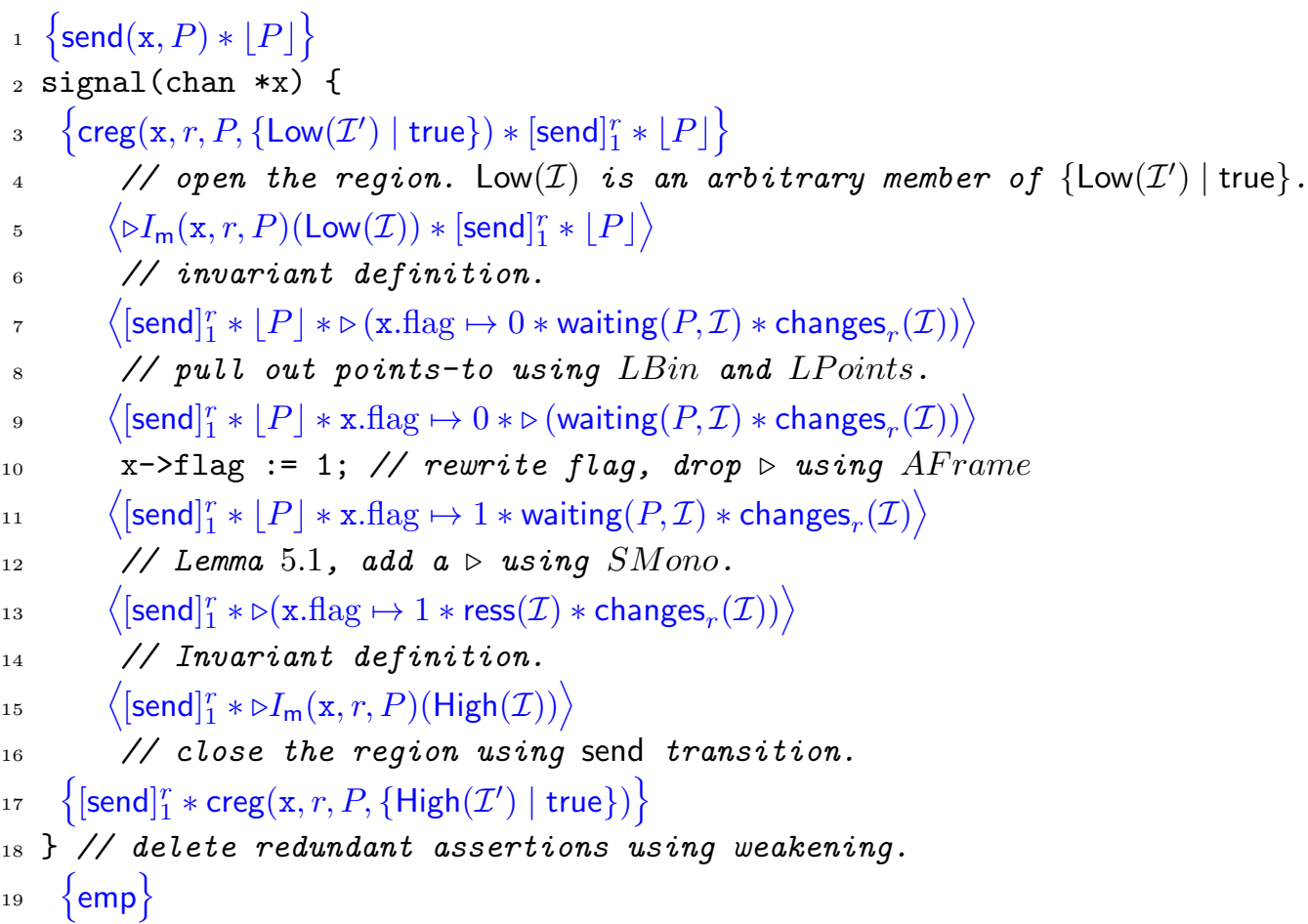

Fig. 14. Proof of signal() w.r.t. the full specification.

line 6 - creating the stabilised assertion - and line 10 - the view-shift which creates the region itself. In line 6 , we need the following implication:

$$
\mathrm{emp} \Longrightarrow\lfloor(\triangleright P) *(\triangleright P)\rfloor
$$

To show this holds, we observe that for any $X$, emp $\Longrightarrow X * X$, and that emp is always stable. The implication then follows by monotonicity of explicit stabilisation brackets, $(A \Rightarrow$ $B) \Longrightarrow(\lfloor A\rfloor \Rightarrow\lfloor B\rfloor)$.

Line 10 requires us to prove the following view-shift:

$$
\begin{aligned}
r_{1} & \stackrel{1 / 2}{\Longleftrightarrow} P * \mathrm{x} \mapsto 0 *\lfloor(\triangleright P) *(\triangleright P)\rfloor \\
& \sqsubseteq \exists r_{2} \cdot \operatorname{creg}\left(\mathrm{x}, r_{2}, P,\left\{\operatorname{Low}\left(\left\{r_{1}\right\}, \emptyset\right)\right\}\right) *[\operatorname{send}]_{1}^{r_{2}} *\left[\operatorname{change}\left(r_{1}\right)\right]_{1}^{r_{2}}
\end{aligned}
$$

To prove this, we appeal to iCAP's VALLOC rule, which controls construction of new regions (see $\S 4.1$ for its definition). Intuitively, this requires that the resources available satisfy the initial abstract state, defined by $I_{\mathrm{m}}$. Note that all the change tokens apart from change $\left(r_{1}\right)$ are stored in the token library predicate changes, held inside the new region.

Proving signal. The proof of signal (Fig. 14) works by opening the channel region (line 4), merging in the supplied resource $\lfloor P\rfloor$ to give the promised resources (line 12), and closing the region again (line 16). When we close the region we also need to confirm that the transition from $\operatorname{Low}(\mathcal{I})$ to $\operatorname{High}(\mathcal{I})$ is allowed, but this is simple: it's the only transition associated to send by $T_{\mathrm{m}}$. The trickiest step is the merging of the resource into the region (line 12), embodied by the following lemma.

LEMMA 5.1. $\lfloor P\rfloor *$ waiting $(P, \mathcal{I}) \sqsubseteq \operatorname{ress}(\mathcal{I})$ 


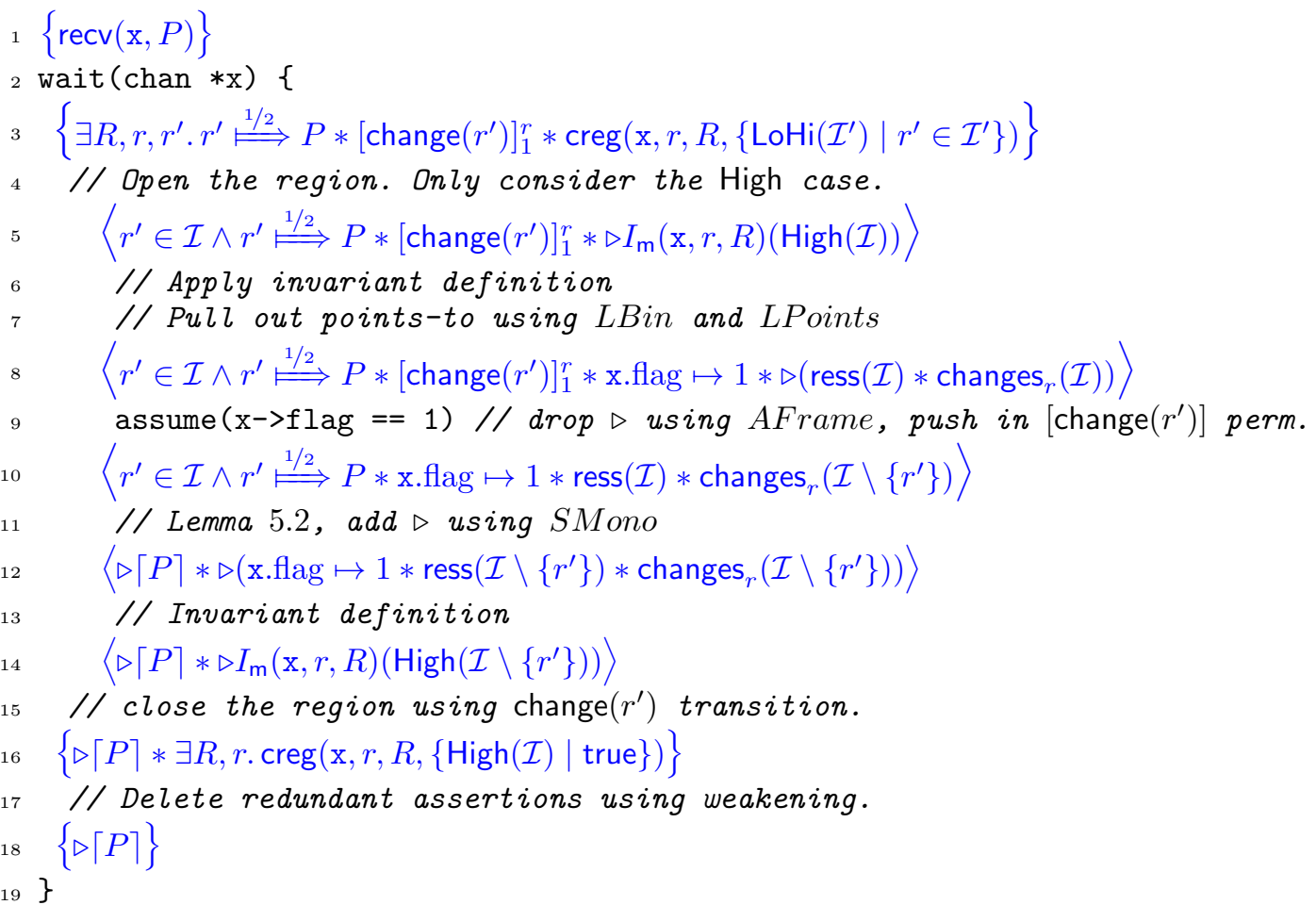

Fig. 15. Proof of wait() w.r.t. the full specification.

Proof. $\lfloor P\rfloor *$ waiting $(P, \mathcal{I})$

$$
\begin{aligned}
& \sqsubseteq\lfloor\triangleright P\rfloor * \exists Q: \mathcal{I} \rightarrow \text { Prop. }\left\lfloor\triangleright P \rightarrow \triangleright \circledast_{i \in \mathcal{I}} \cdot Q(i)\right\rfloor *\left(\circledast_{i \in \mathcal{I}} \cdot i \stackrel{1 / 2}{\Longrightarrow} Q(i)\right) \\
& \sqsubseteq \exists Q: \mathcal{I} \rightarrow \text { Prop. }\left\lfloor\triangleright \circledast_{i \in \mathcal{I}} \cdot Q(i)\right\rfloor *\left(\circledast_{i \in \mathcal{I}} \cdot i \stackrel{1 / 2}{\Longrightarrow} Q(i)\right) \\
& \sqsubseteq \exists Q: \mathcal{I} \rightarrow \text { Prop. }\left(\circledast_{i \in \mathcal{I}} \cdot\lceil\triangleright Q(i)\rceil\right) *\left(\circledast_{i \in \mathcal{I}} \cdot i \stackrel{1 / 2}{\Longrightarrow} Q(i)\right) \\
& \sqsubseteq\left(\circledast_{i \in \mathcal{I}} \cdot \exists R \cdot i \stackrel{1 / 2}{\Longleftrightarrow} R *\lceil\triangleright R\rceil\right) \\
& \sqsubseteq \operatorname{ress}(\mathcal{I})
\end{aligned}
$$

All of the steps in this proof are in fact standard implications (this is also true of all the other view-shift lemmas unless stated explicitly). To prove the second step, we appeal to the fact that $\lfloor-\rfloor$ is semi-distributitive over separating conjunction, $\lfloor A\rfloor *\lfloor B\rfloor \Longrightarrow\lfloor A * B\rfloor$, and modus ponens for separating implication, $A *(A * B) \Longrightarrow B$. The third step follows from the fact that $\lceil-\rceil$ is weaker than $\lfloor-\rfloor$, and $\lceil-\rceil$ is semi-distributitive over separating conjunction, $\lceil A * B\rceil \Longrightarrow\lceil A\rceil *\lceil B\rceil$.

Proving wait. In the proof of wait (Fig. 15) we open the shared region (line 4), extract the required resource (line 11) and close the region again (line 15). For simplicity, we assume that the abstract state is High; if not, the algorithm spins doing nothing until it is the case. Each promised resource is associated with a region identifier $i$ in the set $\mathcal{I}$; removing the resource is modelled abstractly by removing $i$. This abstract transition is allowed by the [change] permission. The key step in the proof is extracting the resource (line 11), embodied by the following lemma. 


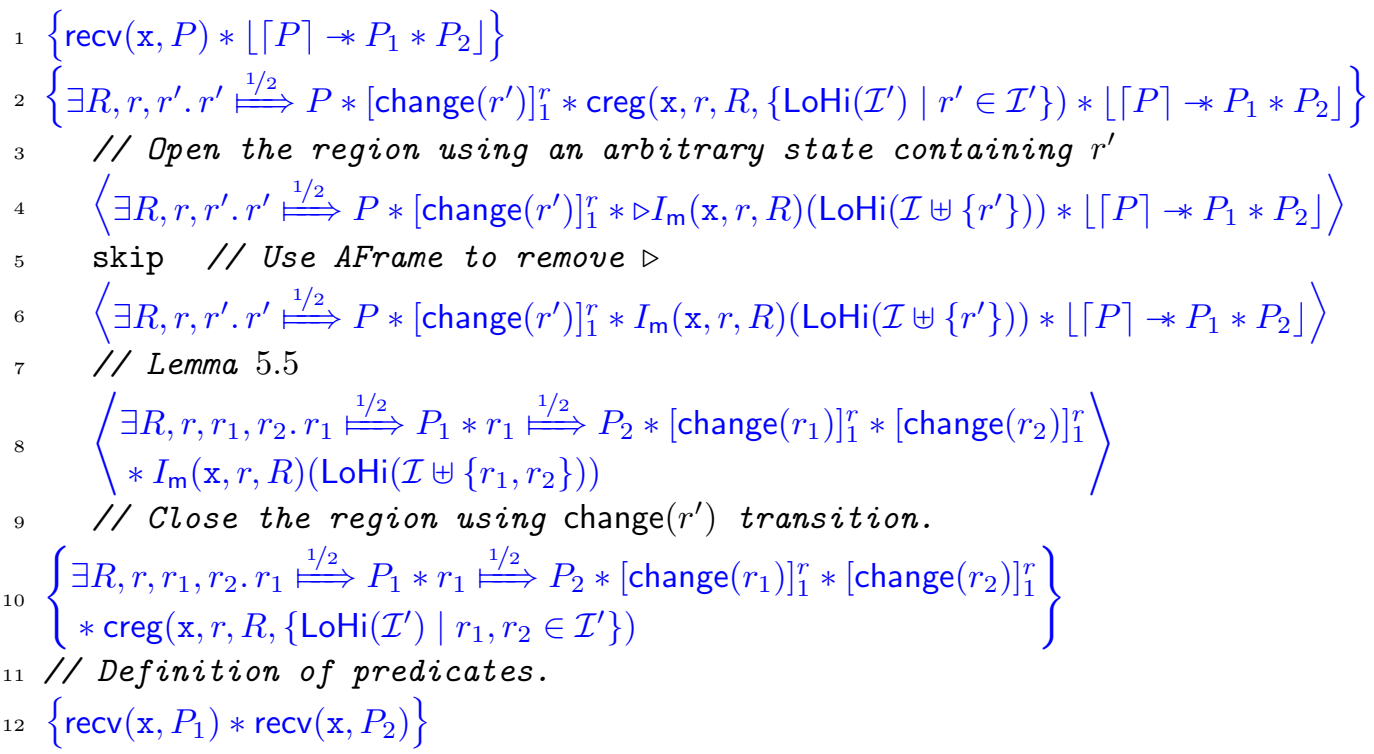

Fig. 16. Proof outline for splitting axiom.

LEMMA 5.2. $\quad r \stackrel{1 / 2}{\Longleftrightarrow} P * \operatorname{ress}(\mathcal{I}) \wedge r \in \mathcal{I} \sqsubseteq \operatorname{ress}(\mathcal{I} \backslash\{r\}) * \triangleright\lceil P\rceil$

Proof. $r \stackrel{1 / 2}{\Longleftrightarrow} P * \operatorname{ress}(\mathcal{I} \uplus\{r\})$

$$
\begin{array}{lll}
\sqsubseteq r \stackrel{1 / 2}{\Longleftrightarrow} P * \operatorname{ress}(\mathcal{I}) * \exists R . r \stackrel{1 / 2}{\Longleftrightarrow} R *\lceil\triangleright R\rceil & & \text { (Definition of ress) } \\
\sqsubseteq r \stackrel{1 / 2}{\Longleftrightarrow} P * \operatorname{ress}(\mathcal{I}) * \exists R . r \stackrel{1 / 2}{\Longleftrightarrow} R *\lceil\triangleright P\rceil & & \text { (Property } 3, \text { mono of }\lceil-\rceil) \\
\sqsubseteq \operatorname{ress}(\mathcal{I}) * \triangleright\lceil P\rceil & & (\lceil\triangleright P\rceil \Longrightarrow \triangleright\lceil P\rceil)
\end{array}
$$

Proving the Splitting Axiom. In our specification, splitting must always be associated with a skip step. It should now be clear why we need this: a skip step allows us to enter the shared region and get rid of $\triangleright$. We present the proof outline in Figure 16. The core of the proof is two lemmas which express splitting in the Low and High cases.

Lemma 5.3 (LOW SPlitTing).

$$
\begin{aligned}
& r \stackrel{1 / 2}{\Longleftrightarrow} P *\left\lfloor\lceil P\rceil * P_{1} * P_{2}\right\rfloor * \text { waiting }(R, \mathcal{I} \uplus r) \\
& \sqsubseteq \exists r_{1}, r_{2} . r_{1} \stackrel{1 / 2}{\Longrightarrow} P_{1} * r_{2} \stackrel{1 / 2}{\Longleftrightarrow} P_{2} * \text { waiting }\left(R, \mathcal{I} \uplus\left\{r_{1}, r_{2}\right\}\right)
\end{aligned}
$$


Proof.

$$
\begin{aligned}
& r \stackrel{1 / 2}{\Longleftrightarrow} P *\left\lfloor\lceil P\rceil * P_{1} * P_{2}\right\rfloor * \text { waiting }(R, \mathcal{I} \uplus r) \\
& \text { (Predicate definitions, extract saved propositions) } \\
& \sqsubseteq \exists Q^{\prime} . r \stackrel{1 / 2}{\Longleftrightarrow} P * r \stackrel{1 / 2}{\Longleftrightarrow} Q^{\prime} *\left\lfloor\lceil P\rceil * P_{1} * P_{2}\right\rfloor * \\
& \exists Q: \mathcal{I} \rightarrow \text { Prop. }\left\lfloor(\triangleright R) * \triangleright Q^{\prime} * \triangleright \circledast_{i \in \mathcal{I}} \cdot Q(i)\right\rfloor *\left(\circledast_{i \in \mathcal{I}} \cdot i \stackrel{1 / 2}{\Longleftrightarrow} Q(i)\right) \\
& \sqsubseteq\left\lfloor\lceil\triangleright P\rceil *\left(\triangleright P_{1}\right) *\left(\triangleright P_{2}\right)\right\rfloor * \\
& \text { (Unify } P / Q^{\prime} \text {, apply } P \Longrightarrow\lceil P\rceil \text { ) } \\
& \exists Q: \mathcal{I} \rightarrow \text { Prop. }\left\lfloor(\triangleright R) *\lceil\triangleright P\rceil * \triangleright \circledast_{i \in \mathcal{I}} \cdot Q(i)\right\rfloor *\left(\circledast_{i \in \mathcal{I}} \cdot i \stackrel{1 / 2}{\Longrightarrow} Q(i)\right) \\
& \exists Q: \mathcal{I} \rightarrow \text { Prop. }\left\lfloor(\triangleright R) *\left(\triangleright P_{1}\right) *\left(\triangleright P_{2}\right) * \triangleright \circledast_{i \in \mathcal{I}} \cdot Q(i)\right\rfloor *\left(\circledast_{i \in \mathcal{I}} \cdot i \stackrel{1 / 2}{\Longleftrightarrow} Q(i)\right)
\end{aligned}
$$

Lemma 5.4 (High SPlitting).

$$
\begin{aligned}
& r \stackrel{1 / 2}{\Longleftrightarrow} P *\left\lfloor\lceil P\rceil * P_{1} * P_{2}\right\rfloor * \operatorname{ress}(\mathcal{I} \uplus r) \\
& \sqsubseteq \exists r_{1}, r_{2} \cdot r_{1} \stackrel{1 / 2}{\Longrightarrow} P_{1} * r_{2} \stackrel{1 / 2}{\Longleftrightarrow} P_{2} * \operatorname{ress}\left(\mathcal{I} \uplus\left\{r_{1}, r_{2}\right\}\right)
\end{aligned}
$$

ProOF.

$$
\begin{aligned}
r \stackrel{1 / 2}{\Longleftrightarrow} P *\left\lfloor\lceil P\rceil * P_{1} * P_{2}\right\rfloor * \operatorname{ress}(\mathcal{I} \uplus r) \\
\sqsubseteq\left(\lceil P\rceil * P_{1} * P_{2}\right) * \operatorname{ress}(\mathcal{I}) * \triangleright\lceil P\rceil \\
\sqsubseteq\left((\triangleright\lceil P\rceil) *\left(\triangleright P_{1}\right) *\left(\triangleright P_{2}\right)\right) * \operatorname{ress}(\mathcal{I}) * \triangleright\lceil P\rceil \\
\sqsubseteq \operatorname{ress}(\mathcal{I}) *\left(\triangleright P_{1}\right) *\left(\triangleright P_{2}\right) \\
\sqsubseteq \operatorname{ress}(\mathcal{I}) *\left(\triangleright P_{1}\right) *\left(\triangleright P_{2}\right) * \exists r_{1}, r_{2} . r_{1} \stackrel{1}{\models} P_{1} * r_{2} \supseteqq P_{2} \\
\sqsubseteq \operatorname{ress}\left(\mathcal{I} \uplus\left\{r_{1}, r_{2}\right\}\right) * \exists r_{1}, r_{2} . r_{1} \stackrel{1 / 2}{\Longleftrightarrow} P_{1} * r_{2} \stackrel{1 / 2}{\Longleftrightarrow} P_{2}
\end{aligned}
$$

The last step in building the new ress predicate consists of two applications of the following sub-lemma:

$$
\begin{aligned}
& \operatorname{ress}(\mathcal{I}) * \triangleright P * r \stackrel{1}{\models} P \\
& \sqsubseteq\left(\circledast_{i \in \mathcal{I}} \exists Q . i \stackrel{1 / 2}{\longmapsto} Q *\lceil\triangleright Q\rceil\right) *\lceil\triangleright P\rceil * r \stackrel{1}{\models} P \\
& \sqsubseteq\left(\circledast_{i \in \mathcal{I} \uplus\{r\}} \exists Q \cdot i \stackrel{1 / 2}{\Longleftrightarrow} Q *\lceil\triangleright Q\rceil\right) * r \stackrel{1 / 2}{\Longleftrightarrow} P \\
& \sqsubseteq \operatorname{ress}(\mathcal{I} \uplus\{r\}) * r \stackrel{1 / 2}{\Longleftrightarrow} P
\end{aligned}
$$

These two lemmas are combined as follows. 
to the shared channel by the thread and environment are represented by transitions over these abstract states. Predicates send, recv are defined as constraints on the abstract state.

The difference with the new implementation is that operations access multiple channels along the chain. As a result, the abstract state cannot be a single channel: instead it is an ordered sequence of channel nodes from the set CNode:

$$
[\operatorname{node}(x), \operatorname{node}(y), \operatorname{node}(z), \ldots]
$$

Here $x, y, z$ are addresses, and channel nodes are ordered $z \prec y \prec x$. Note that the list is reversed with respect to chain order: nodes closer to the tail precede than those closer to the head. (We do this because pointers in the underlying list go in this direction).

Each CNode plays a similar role to an individual channel in the previous section. Therefore, each has a state High / Low, and a set $\mathcal{I}$ representing splittings of the promised resource. In addition, to handle renunciation each node records a set $\mathcal{W}$ of identifiers for resources promised to it through renunciation. Formally, channel nodes have the following structure:

$\begin{array}{ll}\text { CNode } \triangleq\langle & \\ \text { loc } \in \text { Addr, } & \text { (physical address) } \\ \text { res } \in \mathrm{RId}, & \text { (region ID for sent resource) } \\ \mathcal{I} \in \mathcal{P}_{\text {fin }}(\mathrm{RId}), & \text { (region IDs for promised resources) } \\ \text { flg } \in\{\text { High, Low }\}, & \text { (flag status) } \\ \mathcal{W} \in \mathcal{P}_{\text {fin }}(\mathrm{RId}), & \text { (region IDs for earlier renounced resources) } \\ & \end{array}$

Each CNode represents one channel in the chain, so the abstract state of the barrier is an abstract chain consisting of a finite sequence in $\mathrm{CNode}^{+}$.

We assume that the CNode locations in any abstract chain are pairwise-distinct. Thus, where convenient we sometimes treat an abstract chain as a function from locations to tuples: i.e. $r s(x)$ gives some tuple $(r, \mathcal{I}, f, \mathcal{W})$. Given a CNode $s$, we sometimes write $s$. $f l g$, $s . \mathcal{I}$ etc. to identify the appropriate components of the tuple. We use 0 and 1 to represent the Low and High flag state, respectively.

Most of the abstract transitions are just operations on individual channels in the chain: these are liftings of the single-channel operations defined the previous section. For example, the operation set just involves rewriting the flag field for some channel from Low to High, leaving the state otherwise unchanged.

Renunciation is the most interesting case as it involves two channels in the chain. The abstract specification for renunciation uses a resource from a recv predicate to satisfy a send predicate - the former must be earlier in the chain than the latter. Correspondingly, the renun abstract transition copies an identifier from the earlier channel's promise set, to the later channel's renunciation set. For example:

$$
\begin{aligned}
{\left[\ldots\langle x, i, \mathcal{I}, 0, \mathcal{W}\rangle \ldots\left\langle y, i^{\prime}, \mathcal{I}^{\prime} \uplus\{r\}, 0, \mathcal{W}^{\prime}\right\rangle \ldots\right] } & \leadsto \\
& {\left[\ldots\left\langle x, i^{\prime \prime}, \mathcal{I}, 0, \mathcal{W} \uplus\{r\}\right\rangle \ldots\left\langle y, i^{\prime}, \mathcal{I}^{\prime} \cup\{r\}, 0, \mathcal{W}^{\prime}\right\rangle \ldots\right] }
\end{aligned}
$$

In the proof, there will exist saved propositions $r \stackrel{1}{\models} P$ and $i \stackrel{1}{\models} Q$ - the former records the promised resource from $y$, while the latter records the resource required to signal the channel $x$. Intuitively, after this transition, the resource $P$ can no longer be claimed: it will be used to satisfy channel $x$. This corresponds to the send predicate disappearing in the renunciation axiom. The identifier $i$ also changes to $i^{\prime \prime}-$ the associated saved proposition will now be $i^{\prime \prime} \stackrel{1}{\models}(P * Q)$, refecting the fact that $P$ no longer needs to be supplied. 
This transition is purely abstract in the same way that splitting is: nothing has changed in the concrete representation. All that has changed is the way that the threads agree to use resources.

Chain extension also involves multiple channels. The abstract specification takes as its precondition a send and two sets $E$ and $L$ representing nodes earlier and later in the chain:

$$
\left\{\begin{array}{l}
\operatorname{send}(\mathrm{x}, P) * \\
\circledast_{e \in E} e \prec \mathrm{x} * \circledast_{l \in L} \mathrm{x} \prec l
\end{array}\right\} \quad(\mathrm{b}, \mathrm{a})=\operatorname{extend}(\mathrm{x})\left\{\begin{array}{l}
\operatorname{send}(\mathrm{b}, Q) * \operatorname{recv}(\mathrm{b}, Q) * \operatorname{send}(\mathrm{a}, P) \\
* \mathrm{~b} \prec \mathrm{a} * \circledast_{e \in E} e \prec \mathrm{b} * \circledast_{l \in L} \mathrm{a} \prec l
\end{array}\right\}
$$

In the abstract state, this corresponds to the following transition:

$$
\begin{aligned}
& {[\ldots \text { nodes in } L \ldots\langle\mathrm{x}, i, \mathcal{I}, 0, \mathcal{W}\rangle \ldots \text { nodes in } E \ldots]} \\
& \quad\left[\ldots \text { nodes in } L \ldots\langle\mathrm{a}, i, \mathcal{I}, 0, \mathcal{W}\rangle,\left\langle\mathrm{b}, i^{\prime}, \mathcal{I}^{\prime}, 0, \emptyset\right\rangle \ldots \text { nodes in } E \ldots\right]
\end{aligned}
$$

The new node is inserted immediately preceding the parameter $\mathrm{x}$, with the remaining structure of the abstract state remaining unchanged.

\subsection{Definitions and predicates}

Abstract state predicates. The introduction of renunciation makes it important that resources used later in the chain are available as promised earlier in the chain. More concretely, given an abstract chain $x \cdot x s$, every identifier in the set $x . \mathcal{W}$ must be available from some node in $x s$ (i.e. in some set $\mathcal{I}$ ):

$$
\begin{aligned}
& \text { available }([]) \triangleq \emptyset, \quad \text { available }(s \cdot x s) \triangleq(\text { available }(x s) \backslash s . \mathcal{W}) \uplus s . \mathcal{I} \\
& w f([]) \triangleq \text { true, } \quad \operatorname{wf}(s \cdot x s) \triangleq \operatorname{wf}(x s) \wedge s \cdot \mathcal{W} \subseteq \text { available }(x s) \wedge s \cdot \mathcal{I} \cap s . \mathcal{W}=\emptyset \wedge \\
& \forall s^{\prime} \in x s . s . \mathcal{I} \cap s^{\prime} \cdot \mathcal{I}=\emptyset \wedge s . \mathcal{W} \cap s^{\prime} \cdot \mathcal{W}=\emptyset
\end{aligned}
$$

The predicate available constructs the set of identifiers that have been promised earlier in the chain, and that have not been taken by some other earlier channel. Well-formedness, wf, then requires that the set of identifiers available from earlier in the chain includes those required by the current channel.

We also define two predicates over abstract chains, ctrue and cconf. The first asserts that all the flags in the abstract chain have been set, while the second furthermore asserts that all sets of waited-for renounced resources are empty. When the latter holds, any resources promised by this node must be available for retrieval.

$$
\begin{aligned}
& \operatorname{ctrue}(r s) \triangleq \forall e \in r s . e . f l g=1 \\
& \operatorname{cconf}(r s) \triangleq \forall e \in r s . e . f l g=1 \wedge e . \mathcal{W}=\emptyset
\end{aligned}
$$

Finally, we use $r s_{1} \stackrel{p r}{\rightarrow} r s_{2}$ to denote that the abstract chain $r s_{2}$ can be derived from $r s_{1}$ by cancelling out renounced resources with the corresponding promises. This is used in the proof to show that renounced resources can eventually be satisfied with real resources, once all the flags in the chain have been set. $\stackrel{p r}{\rightarrow}^{*}$ is the transitive-reflexive closure of a relation which cancels a single promise to a later node using a renounced resource from an earlier node. For example:

$$
\begin{array}{r}
{\left[\ldots\langle x, i, \mathcal{I}, 1, \mathcal{W} \uplus\{r\}\rangle, \ldots\left\langle y, i^{\prime}, \mathcal{I}^{\prime} \uplus\{r\}, 1, \mathcal{W}^{\prime}\right\rangle \ldots\right] \quad \stackrel{p r}{\longrightarrow}} \\
{\left[\ldots\langle x, i, \mathcal{I}, 1, \mathcal{W}\rangle, \ldots\left\langle y, i^{\prime}, \mathcal{I}^{\prime}, 1, \mathcal{W}^{\prime}\right\rangle \ldots\right]}
\end{array}
$$




$$
\begin{aligned}
& \operatorname{renun}_{c}\left(x, r s, r s^{\prime}\right) \triangleq r s(x) . f l g=0 \wedge \exists r^{\prime}, w \cdot r s^{\prime}=\left(r s \boldsymbol{४}_{x} \boldsymbol{\triangleleft}_{r e s} r^{\prime}\right) \boldsymbol{४}_{x} \boldsymbol{४}_{\mathcal{W}}(\bullet \uplus w) \\
& \operatorname{set}_{c}\left(x, r s, r s^{\prime}\right) \triangleq r s(x) . f l g=0 \wedge r s^{\prime}=r s \boldsymbol{४}_{x} \boldsymbol{४}_{f l g}(1) \\
& \operatorname{ext}_{c}\left(x, r s, r s^{\prime}\right) \triangleq r s=\left(r s_{1} \cdot a \cdot r s_{2}\right) \wedge r s^{\prime}=\left(r s_{1} \cdot a \cdot b \cdot r s_{2}\right) \wedge \\
& \text { a.loc }=x \wedge a . f l g=0 \wedge b . f l g=0 \wedge b . \mathcal{W}=\emptyset \\
& \operatorname{split}_{c}\left(x, r, r s, r s^{\prime}\right) \triangleq r \in r s(x) . \mathcal{I} \wedge \exists r_{2}, r_{3} . r s^{\prime}=r s \boldsymbol{४}_{x} \boldsymbol{४}_{\mathcal{I}}\left((\bullet \backslash\{r\}) \uplus\left\{r_{2}, r_{3}\right\}\right) \\
& \operatorname{sat}_{c}\left(r s, r s^{\prime}\right) \triangleq r s=\left(r s_{1} \cdot r s_{2}\right) \wedge r s^{\prime}=\left(r s_{1} \cdot r s_{2}^{\prime}\right) \wedge \operatorname{cconf}\left(r s_{2}^{\prime}\right) \wedge r s_{2} \stackrel{p r}{\rightarrow} r s_{2}^{\prime} \\
& \operatorname{get}_{c}\left(x, r, r s, r s^{\prime}\right) \triangleq r s=\left(r s_{1} \cdot r s(x) \cdot r s_{2}\right) \wedge \operatorname{cconf}\left(r s(x) \cdot r s_{2}\right) \wedge r s^{\prime}=r s \varangle_{x} \triangleleft_{\mathcal{I}}(\bullet \backslash\{r\}) \\
& T_{\mathrm{c}}(\operatorname{send}(x)) \triangleq\left\{(a, b) \mid \operatorname{wf}(b) \wedge\left(\operatorname{renun}_{c}(x, a, b) \vee \operatorname{set}_{c}(x, a, b) \vee \operatorname{ext}_{c}(x, a, b)\right)\right\} \\
& T_{\mathrm{c}}(\operatorname{change}(x, r)) \triangleq\left\{(a, b) \mid \operatorname{wf}(b) \wedge\left(\operatorname{split}_{c}(x, r, a, b) \vee \operatorname{sat}_{c}(a, b) \vee \operatorname{get}_{c}(x, r, a, b)\right)\right\}
\end{aligned}
$$

Fig. 17. Definition of $T_{\mathrm{c}}$, the transition relation for the chained channel implementation.

We define the relation formally as follows:

$$
\begin{aligned}
r s \stackrel{p r}{\longrightarrow} r s^{\prime} \triangleq & \exists x, y, r . \\
& r \in r s(x) . \mathcal{W} \wedge r \in r s(y) . \mathcal{I} \wedge(x, y) \in \operatorname{ord}(r s) \wedge \\
& r s^{\prime}=\left(r s \varangle_{x} \varangle_{\mathcal{W}}(\bullet \backslash\{r\})\right) \varangle_{y} \bullet_{\mathcal{I}}(\bullet \backslash\{r\})
\end{aligned}
$$

We write $(x, y) \in \operatorname{seq}(r s)$ to say that the two addresses $x$ and $y$ appear adjacent in the sequence $r s$, and $(x, y) \in \operatorname{ord}(r s)$ to say just that they are ordered in $r s$.

Lenses. The notation $\triangleleft$ is a lens allowing a single field of a chain to be updated without modifying the remainder of the chain. We use lenses to make our definitions more compact. Lenses are a notation borrowed from functional programming which we use to update one field of an object, while preserving the remainder of it. By object, we mean either a tuple or a map - we treat tuples as maps from field-names to values. Recall that we can also treat abstract chains as maps from locations to tuples as convenient.

We define the lens notation as follows. In the following, let $x$ be the tuple / map we wish to update. Let $i / j$ be values in the domain (i.e field names for a tuple). Let $f$ be an expression with $\bullet$ standing for the value to be updated. Then the lens notation is defined as follows:

$$
x \boldsymbol{\varangle}_{i} f \triangleq x[i \mapsto(f[x(i) / \bullet])] \quad x \boldsymbol{\triangleleft}_{i} \boldsymbol{\triangleleft}_{j} f \triangleq x\left[i \mapsto\left(x(i) \boldsymbol{४}_{j} f\right)\right]
$$

On the left, the value associated with index / field name $f$ in object $x$ is updated to $f[x(i) / \bullet]$. On the right, we show we can stack lenses, allowing us to update fields deeper inside the object - here we update field $j$ of field $i$ of object $x$.

To give an example, in the preceding section, we had the following lens expression:

$$
\left(r s \boldsymbol{\triangleleft}_{x} \boldsymbol{४}_{\mathcal{W}}(\bullet \backslash\{r\})\right) \boldsymbol{४}_{y} \boldsymbol{\triangleleft}_{\mathcal{I}}(\bullet \backslash\{r\})
$$

Note that here we are using $\mathcal{W} / \mathcal{I}$ as identifiers for particular tuple components. This expression denotes the chain $r s$, with identifier $r$ removed from both the set of renounced resources at CNode $x$ and the set of promised resource at CNode $y$.

Predicate definitions. As usual, we begin by defining the structure of the shared region. Abstract states have the form Chain $(r s)$, where $r s$ is an abstract chain. Actions have the form send $(x)$ and change $(x, r)$, where $x$ is an address, and $r$ a region identifier. The transition 


$$
\begin{aligned}
& \operatorname{chainds}(x \cdot y \cdot r s) \triangleq x . l o c \mapsto\{\mathrm{prev}=y . l o c ; \text { flag }=x . f l g\} * \operatorname{chainds}(y \cdot r s) \\
& \operatorname{chainds}(x \cdot \text { null }) \triangleq x . l o c \mapsto\{\text { prev }=\text { NULL; flag }=x . f l g\} \\
& \text { resource }(\mathcal{I}, \mathcal{W}) \triangleq \exists Q: \mathcal{I} \rightarrow \text { Prop, } R: \mathcal{W} \rightarrow \text { Prop } \\
& \circledast_{i \in \mathcal{I}} \cdot i \stackrel{1 / 2}{\Longleftrightarrow} Q(i) * \circledast_{w \in \mathcal{W}} \cdot w \stackrel{1 / 2}{\Longleftrightarrow} R(w) \\
& *\left\lfloor\left(\triangleright \circledast_{w \in \mathcal{W}} \cdot R(w)\right) * \triangleright \circledast_{i \in \mathcal{I}} \cdot\lceil Q(i)\rceil\right\rfloor \\
& \operatorname{chainres}(x \cdot r s) \triangleq \operatorname{resource}(x . \mathcal{I}, x . \mathcal{W} \uplus\{x . r e s \mid \neg x . f l g\}) * \text { chainres }(r s) \\
& \text { chainres }(\text { null }) \triangleq \mathrm{emp} \\
& \mathrm{uS}(r s) \triangleq\left\{x \mid\left(x,_{-},,_{-},,_{-}\right) \in r s\right\} \\
& \mathrm{uC}(r s) \triangleq\left\{(x, i) \mid\left(x,,_{-}, \mathcal{I},,_{-},-\right) \in r s \wedge i \in \mathcal{I} \wedge \neg \exists\left(y,,_{-},,_{-}, \mathcal{W}\right) \in r s . i \in \mathcal{W}\right\} \\
& \text { unused } \left.(r, r s) \triangleq\left(\circledast x \notin \mathrm{uS}(r s) \text {. }[\operatorname{send}(x)]_{1}^{r}\right) *\left(\circledast\left(x, r^{\prime}\right) \notin \mathrm{uC}(r s) \text {. [change }\left(x, r^{\prime}\right)\right]_{1}^{r}\right)
\end{aligned}
$$

Fig. 18. Predicates used in defining the state of a region.

relation $T_{\mathrm{c}}$ is defined in Fig. 17. We assume that physical addresses are used uniquely, so where convenient we use chains as finite functions of type

$$
\text { Addr } \stackrel{\text { fin }}{\longrightarrow}(\text { Rld } \times \mathcal{P}(\text { Rld }) \times\{\text { High, Low }\} \times \mathcal{P}(\text { Rld }))
$$

The transition relation defines six kinds of transitions in Fig. 17. For send we have renunciation, which adds an element to $\mathcal{W}$; setting the flag; and extending the chain, which creates a new CNode $b$. For change we have splitting; satisfying the renounced resource set, which sets $\mathcal{W}$ to $\emptyset$ and pulls the resources out of earlier chain elements; and pulling out a resource.

To translate from an abstract chain to a concrete invariant, we define three predicates: chainds, chainres, and unused (defined in Fig. 18). The predicate chainds represents the linked list underpinning the implementation. Each link in the chain has the appropriate prev and flag values set determined by the corresponding CNode in the chain.

The predicate chainres represents the resources that are communicated through the chain. The key predicate is resource, which ties together a set of promised resources $\mathcal{I}$ and a set of resources waited for $\mathcal{W}$. Note that the set $\mathcal{W}$ of resources waited for includes both renounced resources, and the resource supplied by the preceding channel - these are unioned by chainres. The core of the resource predicate is the following assertion:

$$
\lfloor(\triangleright \underset{w \in \mathcal{W}}{\circledast} \cdot R(w)) * \triangleright \underset{i \in \mathcal{I}}{\circledast} \cdot\lceil Q(i)\rceil\rfloor
$$

Here $Q$ and $R$ map identifiers to propositions. Leaving aside explicit stabilization and $\triangleright$, this assertion has a straightforward intuition: supplying all the resources waited for (those with identifiers in $\mathcal{W}$ ) results in the resources promised (those with identifiers in $\mathcal{I}$ ).

When there are no resources waited for, i.e. $\mathcal{W}=\emptyset$, the resource predicate can be simplified to just the promised resources:

$$
\text { Lemma 6.1. resource }(\mathcal{I}, \emptyset) \sqsubseteq \circledast_{i \in \mathcal{I}} \cdot \exists Q \text { : Prop. } i \stackrel{1 / 2}{\longmapsto} Q(i) *\lceil\triangleright Q(i)\rceil
$$

The unused predicate stands for the set of unused permissions (similar to changes in the previous proof). We define this using uS(rs), the set of used send permissions, and $\mathrm{uC}(r s)$, the set of used change permissions.

The representation function for the region, $I_{\mathrm{c}}$, is defined as follows:

$$
I_{\mathrm{c}}(r)(\text { Chain }(r s)) \triangleq \operatorname{chainds}(r s) * \text { chainres }(r s) * \operatorname{unused}(r, r s)
$$


As before, we use a shorthand for the region assertion in our definitions and proofs:

$$
\operatorname{oreg}(r, S) \triangleq \operatorname{region}\left(S, T_{\mathrm{c}}, I_{\mathrm{c}}(r), r\right)
$$

We can now define the send, recv, and ordering predicates.

$$
\begin{aligned}
& \operatorname{send}(x, P) \triangleq \exists r_{1}, r_{2} . \operatorname{oreg}\left(r_{1},\left\{\text { Chain }(r s) \mid \operatorname{wf}(r s) \wedge r s(x)=\left(r_{2},{ }_{-}, 0_{-}\right)\right\}\right) \\
& * r_{2} \stackrel{1 / 2}{\Longleftrightarrow} P *[\operatorname{send}(x)]_{1}^{r_{1}} \\
& \operatorname{recv}(x, P) \triangleq \exists r_{1}, r_{2} \cdot \operatorname{oreg}\left(r_{1},\left\{\operatorname{Chain}(r s) \mid r s(x)=\left(,, \mathcal{I},_{-},,_{-}\right) \wedge r_{2} \in \mathcal{I} \wedge \operatorname{wf}(r s)\right\}\right) \\
& * r_{2} \stackrel{1 / 2}{\Longleftrightarrow} P *\left[\operatorname{change}\left(x, r_{2}\right)\right]_{1}^{r_{1}} \\
& x \prec y \triangleq \exists r . \operatorname{oreg}(r,\{\text { Chain }(r s) \mid(y, x) \in \operatorname{ord}(r s)\})
\end{aligned}
$$

The structure of the send and recv predicates is similar to the un-chained proof. Both send and recv contain saved propositions for their proposition parameter $P$. The predicate definitions ensure the identifier for this saved proposition is embeddeded into the abstract chain correctly. Meanwhile, the $\prec$ predicate is a straightforward lifting of the ord predicate on abstract chains.

\subsection{Proving signal, wait and extend}

The majority of the proof concerns manipulations of resource obligations, rather than reads and writes to the underlying data-structure. To help with proof clarity, as far as possible we factor reads and writes into small, separate specifications.

Proving signal. The sketch-proof is shown in Fig. 19 - it is similar in structure to the one in $§ 5.3$. The main additional challenge is to show that resources are supplied to the appropriate point in the chain. To do this, we use the following lemma, which says that supplying the resource $\lfloor P\rfloor$ and an associated saved proposition removes the need to supply $r$. This is then sufficient to allow the flag to be set.

Lemma 6.2. $\quad r \stackrel{1 / 2}{\Longleftrightarrow} P *\lfloor P\rfloor * \operatorname{resource}(\mathcal{I}, \mathcal{W} \uplus\{r\}) \sqsubseteq \operatorname{resource}(\mathcal{I}, \mathcal{W})$

Proof. Given in Appendix B.

In the proof of signal, this lemma is used to show that the appropriate resource has been supplied (Fig. 19, line 12). By factoring logical resource transfer away from the physical signalling, we simplify the proof structure considerably.

The rest of the proof consists of manipulating predicates. We pull out the node associated with $\mathrm{x}$ and set the flag (lines 1-8). Once the resource has been supplied on line 12, the remainder of the proof closes the region again.

Proving wait. The sketch-proof is given in Fig. 20. The three most important steps are checking that all preceding flags in the chain are set (lines 5-12), checking that renounced resources have been supplied (line 15), and retrieving the resource from the chain (line 20). The last two of these require helper lemmas, given below.

Resources that are renounced earlier in the chain can be used to satisfy required resources later in the chain. These resources are represented by the set $\mathcal{W}$ in the abstract state of a cnode. Renounced resources need not be supplied when signal is called, but they must be available before wait returns. To ensure this, the implementation of wait checks all the preceding flags in the chain. Once all preceding flags are set, all the resources should be available. However, proving this is subtle, because renounced resources may themselves be satisfied by resources renounced earlier in the chain. 
$1\{\operatorname{send}(\mathrm{x}, P) *\lfloor P\rfloor\}$

2 // Definition of send.

$3\left\{\begin{array}{l}\exists r_{1}, r_{2} \cdot r_{2} \stackrel{1 / 2}{\Longleftrightarrow} P *[\operatorname{send}(\mathrm{x})]_{1}^{r_{1}} *\lfloor P\rfloor * \\ \operatorname{oreg}\left(r_{1},\left\{\text { Chain }(r s) \mid \operatorname{wf}(r s) \wedge r s(\mathrm{x})=\left(r_{2}, \mathcal{I}, 0, \mathcal{W}\right)\right\}\right)\end{array}\right\}$

4 // Enter the region.

${ }_{5}\left\langle\begin{array}{l}\exists r_{1}, r_{2} \cdot r_{2} \stackrel{1 / 2}{\Longleftrightarrow} P *[\operatorname{send}(\mathrm{x})]_{1}^{r_{1}} *\lfloor P\rfloor * \\ \triangleright\left(\text { chainds }(r s) * \text { chainres }(r s) * \operatorname{unused}\left(r_{1}, r s\right) \wedge \operatorname{wf}(r s) \wedge r s(\mathrm{x})=\left(r_{2}, \mathcal{I}, 0, \mathcal{W}\right)\right)\end{array}\right\rangle$

6 // Split up using chain axioms.

$7\left\langle\begin{array}{l}\exists r_{1}, r_{2} \cdot r_{2} \stackrel{1 / 2}{\Longleftrightarrow} P *[\operatorname{send}(\mathrm{x})]_{1}^{r_{1}} *\lfloor P\rfloor * \\ \triangleright\left(\begin{array}{c}\exists r s_{1}, r s_{2}, a . \text { chainds }\left(r s_{1}\right) * \mathrm{x} \mapsto\left\{\mathrm{prev}=\operatorname{hd}\left(r s_{2}\right) . \operatorname{loc} ; \text { flag }=0\right\} * \text { chainds }\left(r s_{2}\right) * \\ \text { chainres }(r s) * \text { unused }\left(r_{1}, r s\right) \wedge r s=r s_{1} \cdot a \cdot r s_{2} \wedge \operatorname{wf}(r s) \wedge a=\left(\mathrm{x}, r_{2}, \mathcal{I}, 0, \mathcal{W}\right)\end{array}\right)\end{array}\right\rangle$

$8 \quad \mathrm{x}->$ flag $=1$; // Set the flag, drop the $\triangleright$ using Aframe.

$/ \exists r_{1}, r_{2} \cdot r_{2} \stackrel{1 / 2}{\Longleftrightarrow} P *[\operatorname{send}(\mathrm{x})]_{1}^{r_{1}} *\lfloor P\rfloor *$

$9\left\langle\exists r s_{1}, r s_{2}, a\right.$. chainds $\left(r s_{1}\right) * \mathrm{x} \mapsto\left\{\mathrm{prev}=\mathrm{hd}\left(r s_{2}\right)\right.$. loc $;$ flag $\left.=1\right\} *$ chainds $\left.\left(r s_{2}\right) *\right\rangle$

chainres $(r s) * \operatorname{unused}\left(r_{1}, r s\right) \wedge r s=r s_{1} \cdot a \cdot r s_{2} \wedge \operatorname{wf}(r s) \wedge a=\left(\mathrm{x}, r_{2}, \mathcal{I}, 0, \mathcal{W}\right)$

10 // Pull out resource predicate from chainres predicate.

$11\left\langle\begin{array}{r}\exists r_{1}, r_{2} \cdot r_{2} \stackrel{1 / 2}{\rightrightarrows} P *[\operatorname{send}(\mathrm{x})]_{1}^{r_{1}} *\lfloor P\rfloor * \\ \exists r s_{1}, r s_{2}, a . \text { chainds }\left(r s_{1}\right) * \mathrm{x} \mapsto\left\{\operatorname{prev}=\operatorname{hd}\left(r s_{2}\right) . l o c ; \text { flag }=1\right\} * \operatorname{chainds}\left(r s_{2}\right) \\ * \operatorname{chainres}\left(r s_{1}\right) * \operatorname{resource}\left(\mathcal{I}, \mathcal{W} \uplus\left\{r_{2}\right\}\right) * \operatorname{chainres}\left(r s_{2}\right) \\ * \operatorname{unused}\left(r_{1}, r s\right) \wedge r s=r s_{1} \cdot a \cdot r s_{2} \wedge \operatorname{wf}(r s) \wedge a=\left(\mathrm{x}, r_{2}, \mathcal{I}, 0, \mathcal{W}\right)\end{array}\right\rangle$

12 // Apply Lemma 6.2 -- use $\lfloor P\rfloor$ to remove $r_{2}$ from the second parameter of resource.

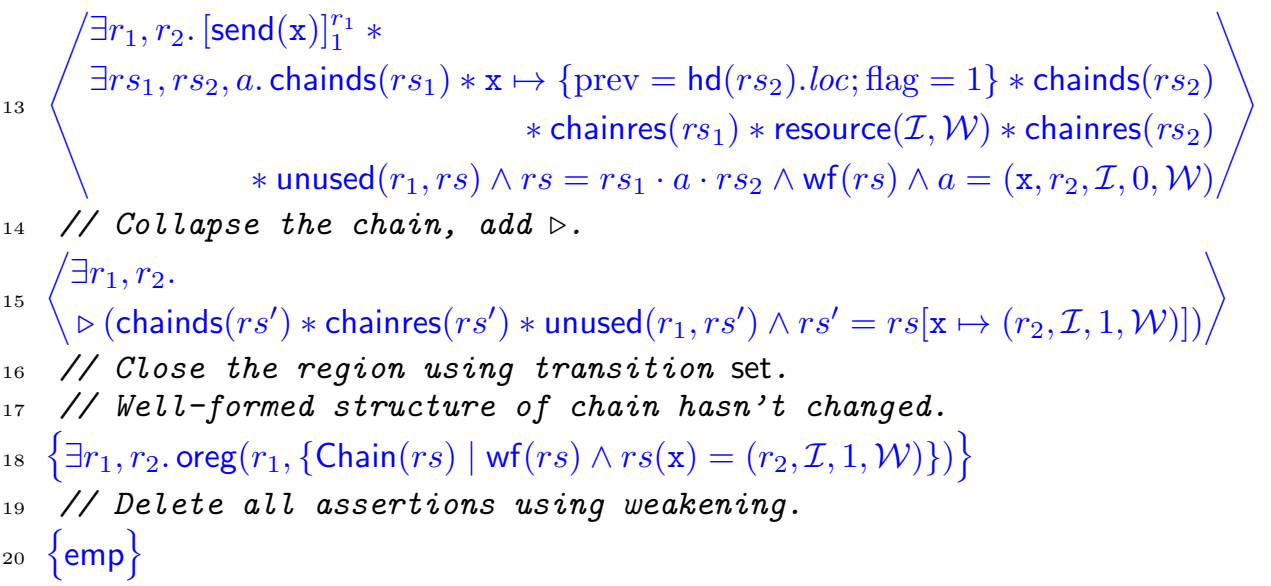

Fig. 19. Sketch-proof for signal with out-of-order signalling. 


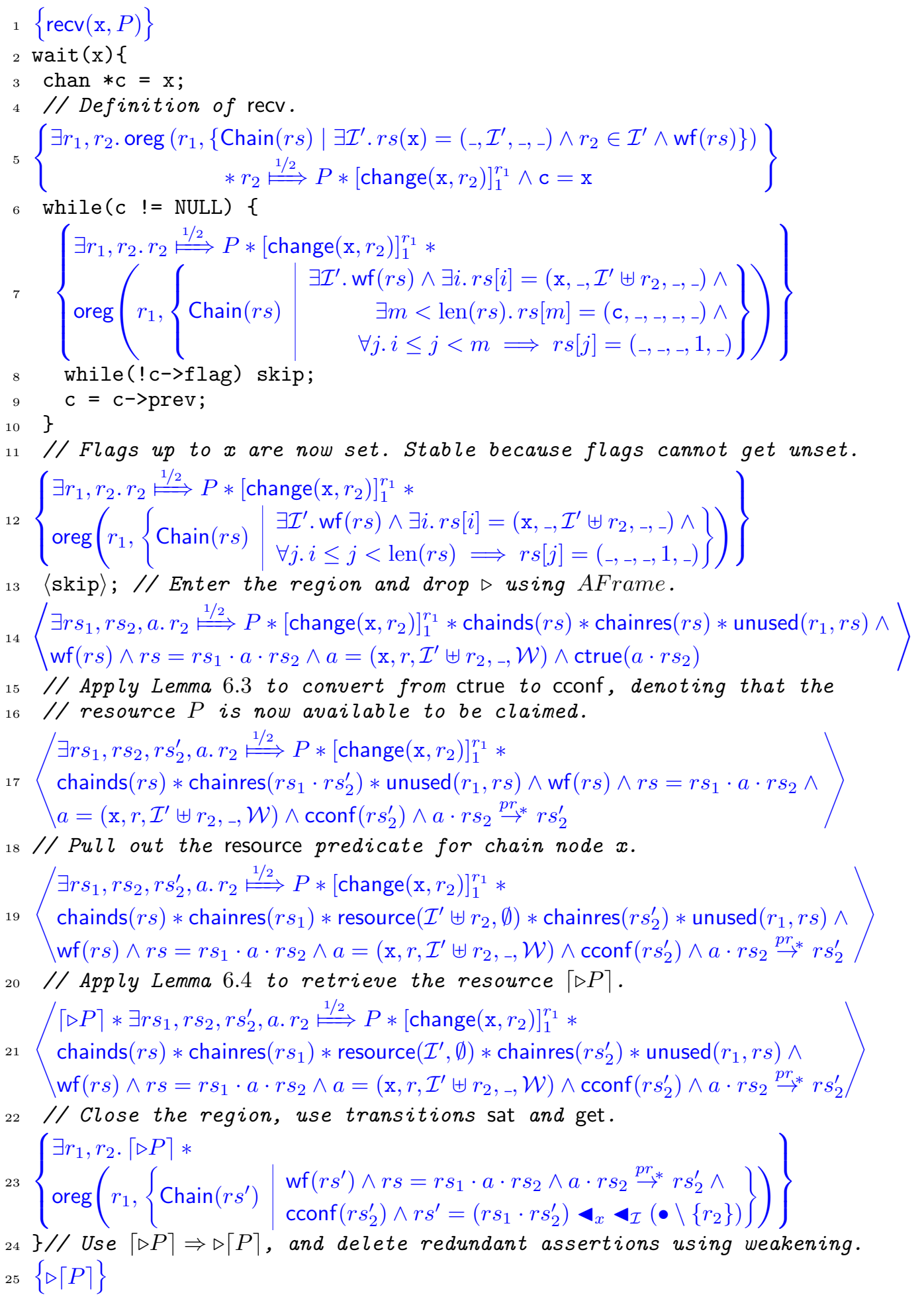

Fig. 20. Sketch-proof for wait with out-of-order signalling. 
To establish the required resources are available, we use the following lemma. This says that a chainres predicate for a chain where all the flags are set can be transformed into one where pending resources have been resolved (asserted by ctrue and cconf respectively).

LEMMA 6.3. chainres $(r s) \wedge \mathrm{wf}(r s) \wedge \operatorname{ctrue}(r s)$

$$
\sqsubseteq \exists r s^{\prime} \text {. chainres }\left(r s^{\prime}\right) \wedge \operatorname{cconf}\left(r s^{\prime}\right) \wedge r s \stackrel{p r}{\rightarrow} r s^{\prime} \wedge \mathrm{wf}\left(r s^{\prime}\right)
$$

Proof. Given in Appendix B.

We apply this lemma on line 15 of the sketch-proof.

Once we've established that the resources are available, we use the following lemma to extract the appropriate resource from the resource predicate:

LEMma 6.4. resource $\left(\mathcal{I} \uplus r_{2}, \emptyset\right) * r_{2} \stackrel{1 / 2}{\Longleftrightarrow} P \sqsubseteq \operatorname{resource}(\mathcal{I}, \emptyset) *\lceil\triangleright P\rceil$

Proof. Given in Appendix B

This lemma says that, given resource and an identifier $r_{2}$ in $\mathcal{I}$ such that all required resources are available, the resource $\triangleright P$ associated with $r_{2}$ can be retrieved. We apply this lemma on line 20 of the sketch-proof.

Proving extend. The sketch-proof is given in Fig. 21. The key steps in the proof are creating a new node to add to the chain (lines 3-9), stitching the new node into the chain itself (line 12), then satisfying the required invariants for the region (lines 18-22).

It is important that new saved propositions are fresh - that is, their identifiers have not been used elsewhere in the chain. We use the following lemma to show new identifiers are fresh:

LEMMA 6.5.

$$
\left\{\operatorname{oreg}(r, \mathcal{T}) * r^{\prime} \stackrel{1}{\Rightarrow} P\right\} \quad\langle\text { skip }\rangle \quad\left\{\operatorname{oreg}\left(r, \mathcal{T} \cap\left\{\text { Chain }(r s) \mid r^{\prime} \notin r s\right\}\right) * r^{\prime} \stackrel{1}{\Rightarrow} P\right\}
$$

Proof. Each identifier $r^{\prime \prime}$ used in $r s$ is associated with a fractional saved proposition $r^{\prime \prime} \stackrel{1 / 2}{\Longleftrightarrow} P$. We case-split on the finite set of possible equalities and appeal to the linearity of saved propositions (Property 2). The skip is required by iCAP because we access the internal state of the region $r$.

The following lemma uses this freshness property, along with the freshness of allocated locations to show that we can we can retrieve the required permissions from unused (the 'library' of unused permissions). We use this lemma on line 16 of the sketch-proof.

LEMMA 6.6 .

$$
\begin{aligned}
& r s=r s_{1} \cdot\left(x, r_{2}, \mathcal{I}, 0, \mathcal{W}\right) \cdot r s_{2} \wedge \text { unused }\left(r_{1}, r s\right) \wedge z, r^{\prime}, r^{\prime \prime} \notin r s \\
& \sqsubseteq \quad \text { unused }\left(r_{1}, r s_{1} \cdot\left(x, r_{2}, \mathcal{I}, 0, \mathcal{W}\right) \cdot\left(z, r^{\prime},\left\{r^{\prime \prime}\right\}, 0, \emptyset\right) \cdot r s_{2}\right) *[\operatorname{send}(z)]_{1}^{r_{1}} *\left[\operatorname{change}\left(z, r^{\prime \prime}\right)\right]_{1}^{r_{1}}
\end{aligned}
$$

Proof. Appeal to the definition of unused.

On line 18, we close the region. The resulting chain is well-formed because the new region has no elements in its renunciation set $\mathcal{W}$, and the rest of the chain is preserved. The chain is stable because we hold the send permission on $x$ and $z$, meaning these channels cannot be extended or renounced.

Proving newchan. Omitted: this proof is similar to extend. However it is simpler: we only need to construct a new point in the chain, and not update the existing chain to take account of it. 


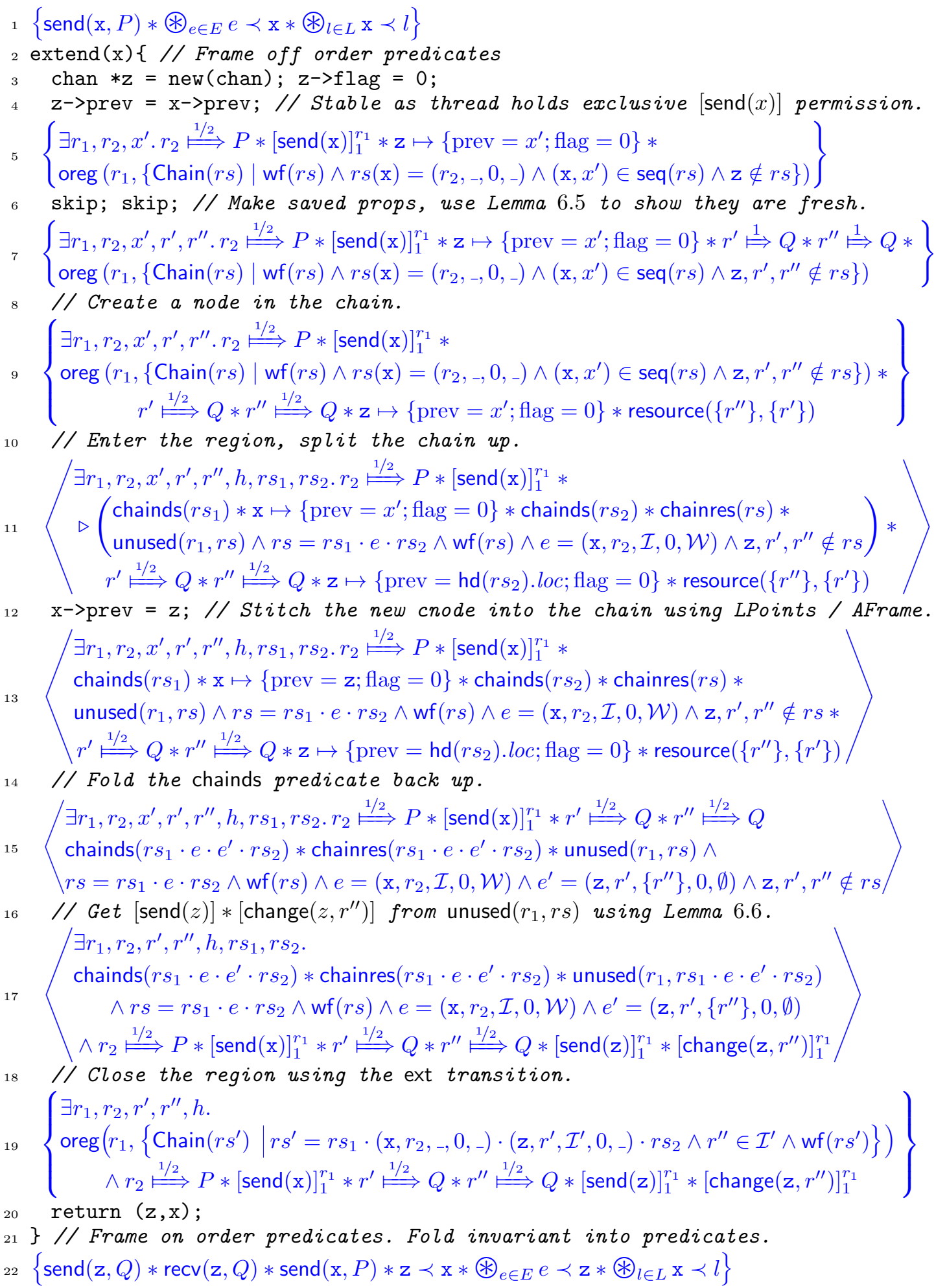

Fig. 21. Sketch-proof of extend with out-of-order signalling. 


\subsection{Proving Renunciation and Splitting Axioms}

Renunciation. The axiom is defined as follows:

$$
\{\operatorname{recv}(x, P) * \operatorname{send}(y, Q) * x \prec y\} \quad\langle\operatorname{skip}\rangle \quad\{\operatorname{send}(y, P * Q)\}
$$

The sketch-proof is given in Fig. 22. Internally each predicate contains a view on the same shared region, and the first step of the proof consists of conjoining these three views to give a single stable view on the shared structure (line 3). The remaining steps are supplying the renounced resource to the shared region (line 13), and closing the region to give a new send predicate (line 15).

In order to conjoin the regions arising from the send, recv and the order predicates, they need to operate over the same region. Although the predicates do not expose region names, we know from order predicates that all of the regions share common elements in their chain addresses. We therefore use an extra lemma to show that pairs of such regions must be the same:

LEMMA 6.7 .

$$
\left\{\operatorname{oreg}(r,\{\text { Chain }(r s) \mid x \in r s\}) * \operatorname{oreg}\left(r^{\prime},\left\{\text { Chain }\left(r s^{\prime}\right) \mid x \in r s^{\prime}\right\}\right)\right\} \quad\langle\operatorname{skip}\rangle \quad\left\{r=r^{\prime}\right\}
$$

Proof. Given in Appendix B.

We use this lemma on line 3, Fig. 22. The conjoined region that arises from this lemma (line 6) is stable because elements cannot be reordered with respect to each other once they are in the chain, and because exclusive [chain] and [send] permissions are held for $x$ and $y$ respectively.

When we push the renounced resource into the resource predicate (line 13) we use the following lemma to show that the renunciation set $\mathcal{W}$ is updated appropriately:

LEMma 6.8 . $\operatorname{resource}(\mathcal{I}, \mathcal{W} \uplus\{r\}) * r \stackrel{1 / 2}{\Longleftrightarrow} Q * r^{\prime} \stackrel{1 / 2}{\Longleftrightarrow} P$

$$
\sqsubseteq \quad \exists r^{\prime \prime} \text {. resource }\left(\mathcal{I}, \mathcal{W} \uplus\left\{r^{\prime}, r^{\prime \prime}\right\}\right) * r^{\prime \prime} \stackrel{1 / 2}{\Longleftrightarrow}(P * Q)
$$

Proof. Given in Appendix B.

Note that the identifier $r$ used for sending resources is replaced with a fresh identifier $r^{\prime \prime}$ because the associated invariant is changed from $Q$ to $P * Q$. Internally this corresponds to deleting one saved proposition through weakening, and then creating another.

On line 15 we close the region. The resulting chain is well-formed because the identifier we selected was previously unused for renunciation - we get this from the definition of unused. Furthermore, the remainder of the chain stays the same. The resulting chain is trivially stable because the exclusive [send] permission is held.

Splitting. The axiom is defined as follows:

$$
\left\{\operatorname{recv}(a, P) *\left\lfloor\lceil P\rceil *\left(P_{1} * P_{2}\right)\right\rfloor\right\} \quad\langle\operatorname{skip}\rangle \quad\left\{\operatorname{recv}\left(a, P_{1}\right) * \operatorname{recv}\left(a, P_{2}\right)\right\}
$$

A sketch-proof is given in Fig. 23. The key step is splitting one element of the promised resource set $\mathcal{I}$ for a node (line 6 ). To do this, we use the following lemma, which states that the saved proposition $r_{2}$ can be exchanged for new saved propositions $r_{3}$ and $r_{4}$ if the appropriate resource $\left\lfloor\lceil P\rceil *\left(P_{1} * P_{2}\right)\right\rfloor$ is supplied.

LEMMA 6.9. $\quad r_{2} \in r s(x) . \mathcal{I} \wedge r_{2} \stackrel{1 / 2}{\Longleftrightarrow} P *\left\lfloor\lceil P\rceil *\left(P_{1} * P_{2}\right)\right\rfloor *$ chainres $(r s)$

$$
\begin{array}{r}
\sqsubseteq \quad \exists r s^{\prime}, r_{3}, r_{4} \cdot r_{3}, r_{4} \notin r s \wedge r s^{\prime}=r s \boldsymbol{४}_{x} \boldsymbol{\cup}_{\mathcal{I}}\left(\left(\bullet \backslash r_{2}\right) \uplus\left\{r_{3}, r_{4}\right\}\right) \wedge \\
r_{3} \stackrel{1 / 2}{\longmapsto} P_{1} * r_{4} \stackrel{1 / 2}{\longmapsto} P_{2} * \text { chainres }\left(r s^{\prime}\right)
\end{array}
$$




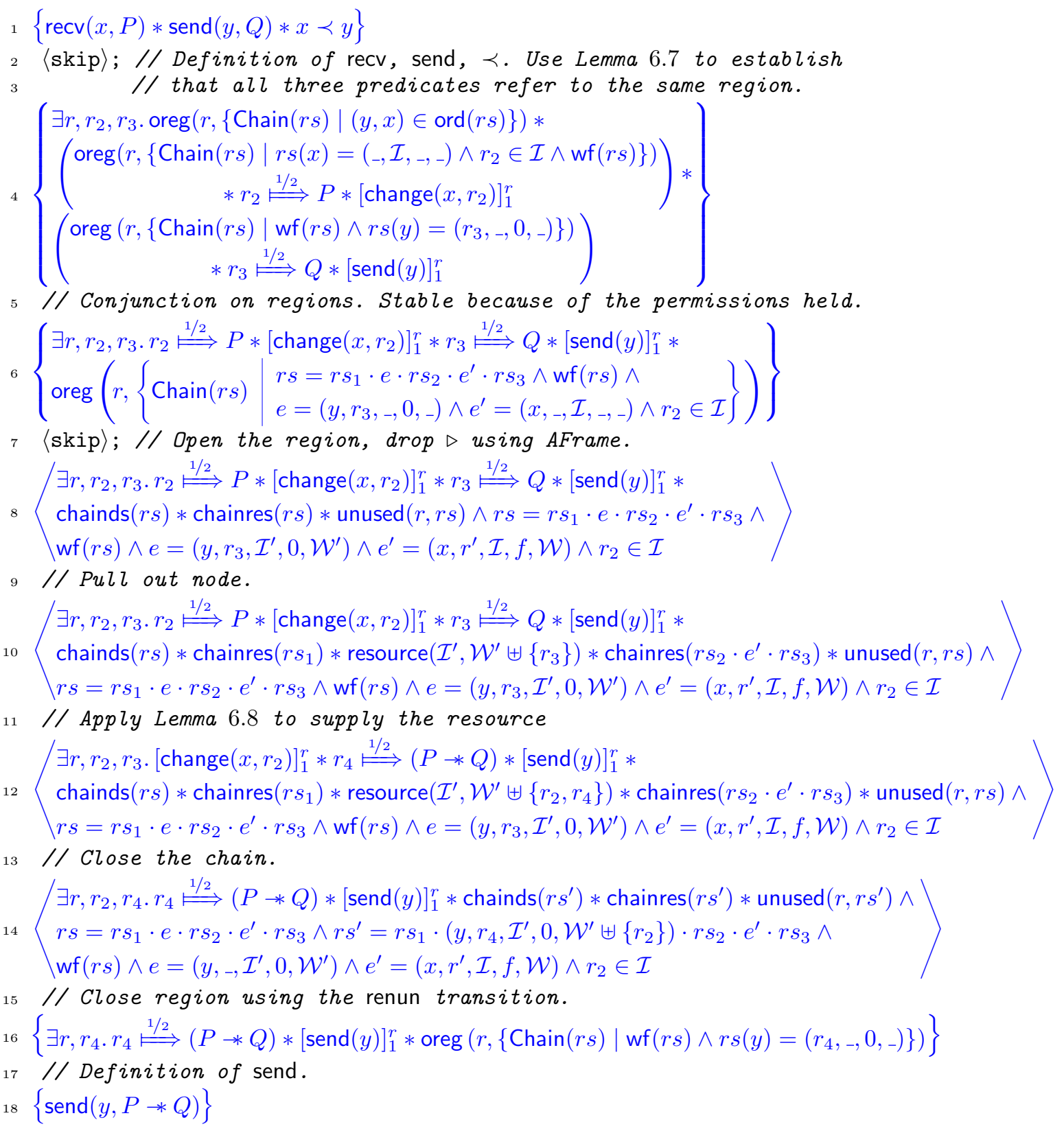

Fig. 22. Proof of the renunciation axiom for out-of-order implementation. 


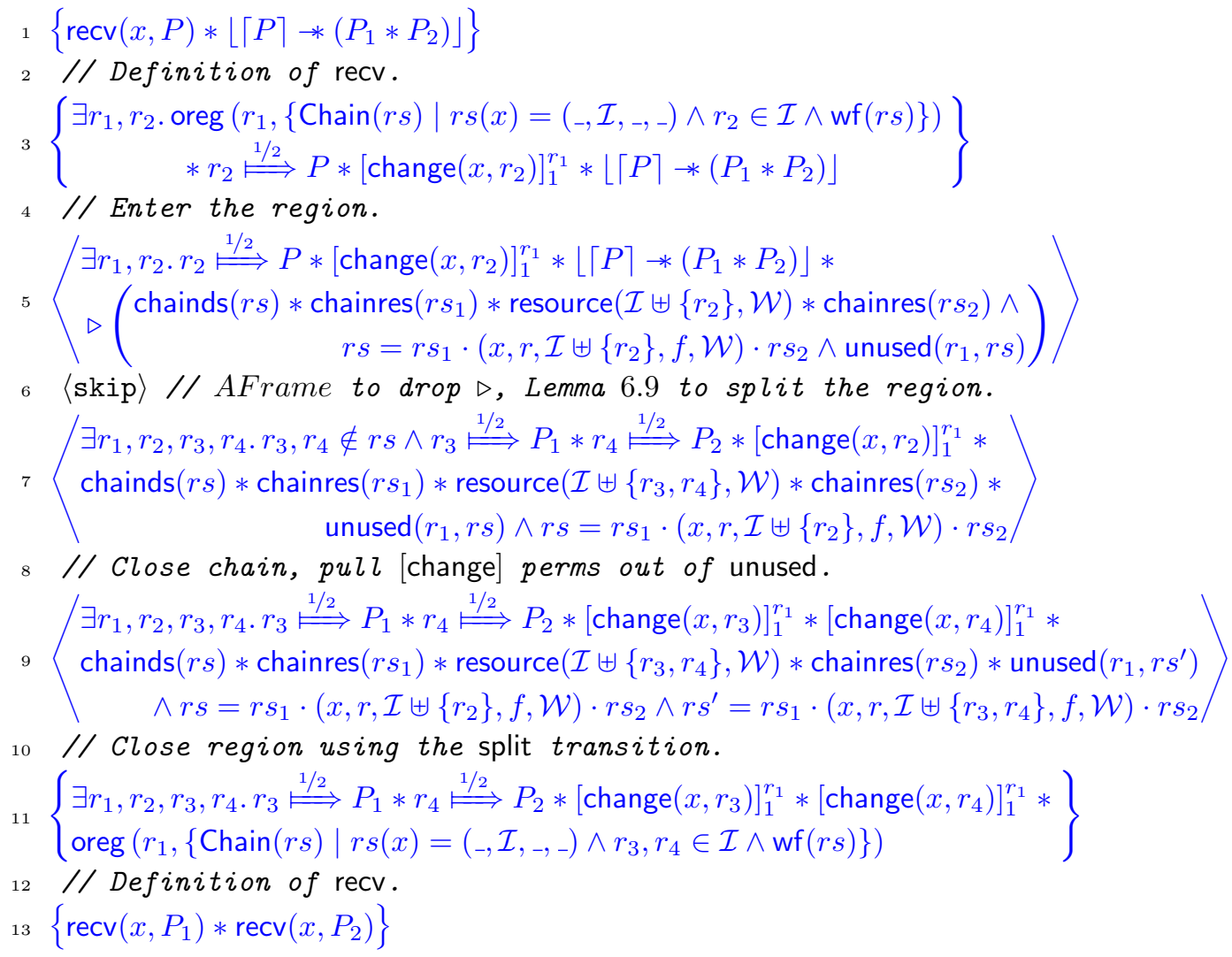

Fig. 23. Proof of the splitting axiom for the out-of-order implementation.

Proof. Given in Appendix B. This proof is very similar in structure to the proof without chaining given in $\S 5.3$.

\section{OUT-OF-ORDER SUMMARIZATION}

In this section we consider our third, most complex, channel implementation. This implementation satisfies our abstract specification, but internally, signals propagate up a tree structure towards a shared root rather than along a linear chain. This barrier implementation corresponds closely to the one in [Navabi et al. 2008] - the summarisation process is how it achieves its efficiency. Verifying this implementation demonstrates that our approach scales to custom synchronisation constructs developed for performance-sensitive concurrency applications.

\subsection{Implementation approach}

Figure 24 shows the implementation. The data-structure is an inverted tree - that is, nodes point upwards towards a single common root. An instance of the data-structure is illustrated in Figure 25.

All of the nodes in the tree are of type chan_addr, and each contains a fixed-size boolean array. Booleans in an array correspond to channel flags, either Low or High. Flags that are leaves, i.e. that do not have a child subtree, represent the channels in the chain. Scanning these leaf flags in order gives the sequence of flags in the abstract chain. 


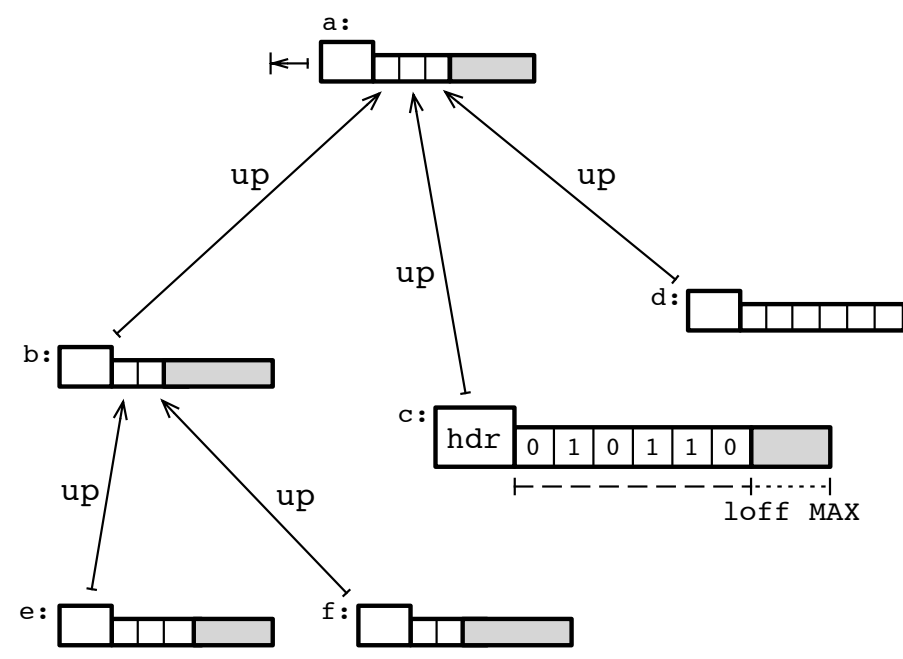

Fig. 25. Example of the summarising channel structure.

to the root. The function waits on each preceding flag in the current array (Fig. 24, lines 6064 - note that increasing the index moves logically earlier in the chain). It then reads the up address stored in the array header and loops if it is not at the root. Because it only ever climbs the tree, the function avoids the cost of iterating over the entire chain. The following diagram shows the nodes accessed when traversing up from $\mathrm{x}$ :

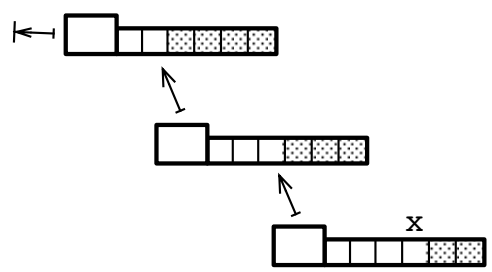

Arrays are allocated with a fixed maximum size MAX, and are gradually filled when extending the chain. The current number of flags active in the array is stored in the header field loff. Thus extend has two cases depending whether there is room in the current array for another leaf (checked on line 17).

- If there is space, then the new leaf is inserted immediately following the current one in the array (lines 20-22).

- If no space remains in the array, then a new array is allocated, and the current flag is used as a summary. The first and second locations in the new array represent the current and newly-created channels (lines 25-31).

As a result of extension, wait may be passed the address of a leaf flag, which is then silently converted into a summary. However, this process is sound: if this summary is set, then the appropriate child flag must also be set (this subtlety is what necessitates the predicate finalleaf in the proof below).

\subsection{Proof Strategy}

Abstract state. At level of specification, the behaviour of the algorithm is unchanged from $\S 6$ - as a result, we can reuse some of the reasoning from there. The algorithm's abstract 
state has two parts: an abstract chain and a heap map. The former dictates the resources promised and renounced at each point in the chain, while the latter defines the underlying inverted-tree pointer structure.

An abstract chain is a sequence in $\mathrm{CNode}^{+}$, defined exactly as in $\S 6$ (see p.38). The only difference is that each node location $l o c$ is now a pair $\langle h, o\rangle$ consisting of a physical address and an offset - this corresponds to the chan_addr type. We reuse abstract chains so that we can reuse reasoning about how resources move through the chain. However, for this implementation an abstract chain alone is insufficient, because it does not represent the underlying inverted tree data-structure.

A heap map represents the inverted-tree structure which controls signal propagation and summarisation. Heap maps are finite, partial functions from physical addresses - each address represents a chan_hdr-typed object forming the tree.

$$
d \in \text { HMap: Addr } \stackrel{\text { fin }}{\longrightarrow}\{u h d r: \text { Addr; uoff : Int; loff : Int; flags: }\{0 . . \mathrm{MAX}\} \rightarrow \text { Bool }\}
$$

(To simplify the representation, we flatten the chan_adr-typed field 'up' into the two subfields $u h d r$ and $u o f f$.

To ensure a heap map represents a correct inverted tree we require several well-formedness properties:

- Each address-offset pair $\langle x, i\rangle$ has at most one child in $d$ which points upward to it. This ensures leaves are uniquely identified.

- Chains of upward pointers are non-cyclic and point towards a common root. This ensures that the data-structure is tree-shaped.

- Flags are only set in non-leaf nodes if all the corresponding leaf nodes are set. This ensures that signals are properly summarised and propagated up the tree.

Given a chain $r s$ and heap map $d$, well-formedness also requires that the two portions of the abstract state correspond. Loosely, this means that the leaves of the inverted tree defined by $d$ are exactly the addresses in the chain $r s$.

Abstract state well-formedness. In order to define formally that $r s$ and $d$ are well-formed and correspond correctly, we require several auxiliary notions:

$$
\begin{aligned}
\operatorname{child}_{d}(x, i, y) & \triangleq d(y) \cdot u h d r=x \wedge d(y) \cdot \text { uoff }=i \wedge 0 \leq i \leq d(x) . \text { loff } \\
\operatorname{leaf}_{d}(\langle x, i\rangle) & \triangleq\langle x, i\rangle \in d \wedge \nexists y \cdot d(y) \cdot \text { uhdr }=x \wedge d(y) \cdot \text { uoff }=i \\
\operatorname{descend}_{d}(\langle x, i\rangle,\langle y, j\rangle) & \triangleq\langle x, i\rangle=\langle y, j\rangle \vee \operatorname{descend}_{d}(\langle x, i\rangle,\langle d(y) \cdot \text { uhdr,d(y).uoff }\rangle) \\
\operatorname{isset}_{d}(\langle x, i\rangle) & \triangleq d(x) \cdot \text { flags }(i)=1 \\
\operatorname{allset}_{d}(x) & \triangleq \forall i .0 \leq i \leq d(x) . \text { loff } \Longrightarrow \operatorname{isset}_{d}(\langle x, i\rangle)
\end{aligned}
$$

(Note we say that a location-offset pair $\langle x, i\rangle$ is in $d$ if $x \in \operatorname{dom}(d) \wedge i \leq d$.loff.)

The child, descend, and leaf predicates record corresponding structural facts about relationships in the tree. Well-formedness on $d$ (defined below) requires that paths through $u h d r$ are finite, which suffices to ensure that descend is well-defined. isset and allset respectively assert that a single address and a whole array have their flags set.

$$
\begin{aligned}
\langle x, i\rangle<_{d}\langle y, j\rangle & \triangleq \exists z, i x, i y \cdot \operatorname{descend}_{d}(\langle z, i x\rangle,\langle x, i\rangle) \wedge \operatorname{descend}_{d}(\langle z, i y\rangle,\langle y, j\rangle) \wedge i x>i y \\
a<_{d}^{\|} b & \triangleq a<_{d} b \vee \operatorname{descend}_{d}(a, b) \vee \operatorname{descend}_{d}(b, a) \\
\text { finalleaf }_{d}(x, y) & \triangleq \operatorname{descend}_{d}(x, y) \wedge \operatorname{leaf}_{d}(y) \wedge\left(\forall z \cdot \operatorname{descend}_{d}(x, z) \wedge \operatorname{leaf}_{d}(z) \Rightarrow z<_{d}^{\|} y\right)
\end{aligned}
$$

The order predicate $<_{d}$ says that two addresses are ordered in the tree, meaning that they share a common ancestor array in which they are also ordered. This defines a transitive and irreflexive order. $<_{d}^{\mathbb{1}}$ says that either two addresses are related by $<_{d}$, or that one is the descendant of the other (i.e. they are on the same path and one summarises the other). 
finalleaf $_{d}(x, y)$ indicates that $y$ is the maximal leaf according to $<_{d}^{\mathbb{t}}$ that is summarised by $x$. This is useful because applications of extend may mean clients wait on $x$ when the actual leaf has been superceded by $y$. For each $x$ there exists at most one $y$ satisfying finalleaf, so we generally use it as a partial function, i.e. finalleaf $f_{d}(x)$ stands for the unique $y$ such that finalleaf $_{d}(x, y)$.

We can now define $\mathrm{wf}(r s, d)$, which requires that $r s$ and $d$ are independently well-formed, and that they are correctly tied together. As abstract chains are unchanged from $\S 6$, we can reuse the prior definition of $\operatorname{wf}(r s)$ (§6.2). For heap-maps, well-formedness is defined as follows:

$$
\begin{aligned}
& \operatorname{wf}(d) \triangleq \exists r: \text { Addr. } \exists \tau: \operatorname{dom}(d) \rightarrow \mathbb{N} . \\
& \forall x, i, y, z .(\langle x, i\rangle \in d \Longrightarrow \exists j \cdot \operatorname{descend}(\langle r, j\rangle,\langle x, i\rangle)) \wedge \\
&\left(\left(\operatorname{child}_{d}(x, i, y) \wedge \operatorname{child}_{d}(x, i, z)\right) \Longrightarrow y=z\right) \wedge \\
&\left(\left(\operatorname{child}_{d}(x, i, y) \wedge \operatorname{isset}_{d}(x, i)\right) \Longrightarrow \operatorname{allset}_{d}(y)\right) \wedge \\
&(d(x) \cdot u h d r=y \wedge y \neq \mathrm{NULL} \Longrightarrow \\
&d(y) \text { defined } \wedge d(x) . u o f f \leq d(y) . \operatorname{loff} \wedge \tau(y)<\tau(x))
\end{aligned}
$$

Here the address $r$ is the location of the tree root, while the function $\tau$ records the distance from the current node to the root - this enforces the absence of cycles. The first clause of the definition ensures all elements in the tree share a common root. The second ensures that children are uniquely identified by address and offset. The third ensures that setting a flag summarises all descendants. The final clause guarantees the existence of non-NULL parents to a node, and enforces the distance function $\tau$.

$$
\begin{aligned}
\operatorname{wf}(r s, d) \triangleq & \operatorname{wf}(r s) \wedge \operatorname{wf}(d) \wedge \\
& \forall r \in r s . r . l o c=\langle l, o\rangle \Longrightarrow r . f l g=d(l) . f l a g s[o] \wedge \\
& \forall r \in r s . \operatorname{leaf}_{d}(r . l o c) \wedge \\
& \forall r_{1}, r_{2} \cdot\left(r s={ }_{-} \cdot r_{1} \cdot{ }_{-} \cdot r_{2} \cdot{ }_{-}\right) \Longrightarrow r_{2} . l o c<_{d} r_{1} \cdot l o c
\end{aligned}
$$

As well as requiring that $r s$ and $d$ are well-formed on their own, this requires (1) that flags in the chain are correctly set in the heap map; (2) locations in the chain are leaves in the heap map; and (3) order in the chain is reflected in the heap-map order $<_{d}$.

Transition map. We define a new transition map $T_{s}$ to capture changes to the heap map (Figure 26). Many of the transitions are inherited from $T_{c}$, the transition relation for the chained implementation $(\S 6.2)$.

set and ext alter the underlying inverted-tree data-structure, and so are defined to allow this. set just updates the tree-map flag appropriately using our lens notation. ext has two cases, reflecting the conditional in the implementation. Either there is enough space in the array to fit another flag, or another array must be added to the heap map. In the latter case, note that the address of the existing channel $a$ is shifted into the new array.

Furthermore, signal can mark summary flags, i.e. flags that are not leaves in the tree; this is allowed by the transition mark. Finally, note finalleaf in the definition of get: this is needed because the real flag may shift its position due to extend, with wait left reading from a summary. Here $x$ is the original flag which has been superceded, and finalleaf ${ }_{d}(x)$ is the current position of the flag. It is safe to pass an address to wait which may have been converted into a summary because extension ensures the original flag must be one of those summarised by $x$.

Interpretation function. For a given map $d$, chainds maps down to the corresponding datastructure definition. Heap maps are intentionally close to the underlying heap: each element 


$$
\begin{aligned}
& \operatorname{renun}_{s}\left(x,(r s, d),\left(r s^{\prime}, d^{\prime}\right)\right) \triangleq d^{\prime}=d \wedge \operatorname{renun}_{c}\left(x, r s, r s^{\prime}\right) \\
& \operatorname{set}_{s}\left(x,(r s, d),\left(r s^{\prime}, d^{\prime}\right)\right) \triangleq x=\langle h, o\rangle \wedge d^{\prime}=d \boldsymbol{\varangle}_{h} \boldsymbol{४}_{\text {flags }[o]}(1) \wedge \operatorname{set}_{c}\left(\langle h, o\rangle, r s, r s^{\prime}\right) \\
& \operatorname{ext}_{s}\left(x,(r s, d),\left(r s^{\prime}, d^{\prime}\right)\right) \triangleq r s=\left(r s_{1} \cdot a \cdot r s_{2}\right) \wedge r s^{\prime}=\left(r s_{1} \cdot b \cdot c \cdot r s_{2}\right) \wedge a . f l g=0 \wedge \\
& \exists y . b=a[l o c \mapsto y] \wedge c . f l g=0 \wedge c . \mathcal{W}=\emptyset \wedge x=\langle h, o\rangle=a . l o c \wedge
\end{aligned}
$$

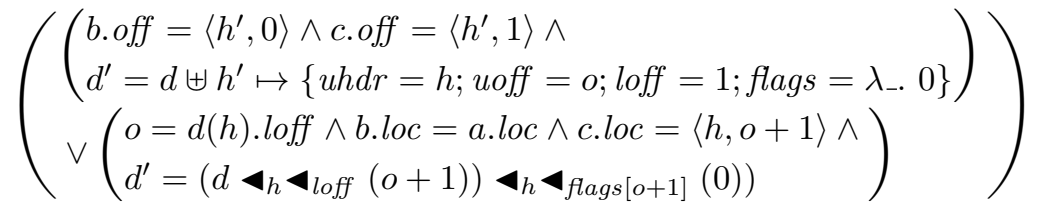

$$
\begin{aligned}
& \operatorname{split}_{s}\left(x, r,(r s, d),\left(r s^{\prime}, d^{\prime}\right)\right) \triangleq d=d^{\prime} \wedge \operatorname{split}_{c}\left(\text { finalleaf }_{d}(x), r, r s, r s^{\prime}\right) \\
& \operatorname{sat}_{s}\left((r s, d),\left(r s^{\prime}, d^{\prime}\right)\right) \triangleq d=d^{\prime} \wedge \operatorname{sat}_{c}\left(r s, r s^{\prime}\right) \\
& \operatorname{get}_{s}\left(x, r,(r s, d),\left(r s^{\prime}, d^{\prime}\right)\right) \triangleq d=d^{\prime} \wedge \operatorname{get}_{c}\left(\text { finalleaf }_{d}(x), r, r s, r s^{\prime}\right) \\
& T_{\mathbf{s}}(\operatorname{send}(x)) \triangleq\left\{(a, b) \mid \operatorname{wf}(b) \wedge\left(\operatorname{renun}_{s}(x, a, b) \vee \operatorname{set}_{s}(x, a, b) \vee \operatorname{ext}_{s}(x, a, b)\right)\right\} \\
& T_{\mathbf{s}}(\operatorname{change}(x, r)) \triangleq\left\{(a, b) \mid \operatorname{wf}(b) \wedge\left(\operatorname{split}_{s}(x, r, a, b) \vee \operatorname{sat}_{s}(a, b) \vee \operatorname{get}_{s}(x, r, a, b)\right)\right\}
\end{aligned}
$$

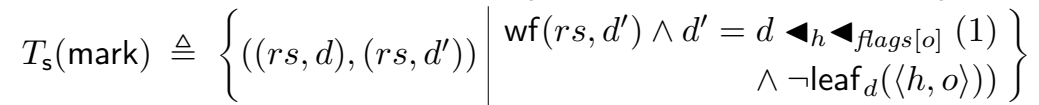

Fig. 26. Definition of the transition relation $T_{\mathrm{s}}$ for the summarising implementation.

in the domain maps to a distinct chan_addr object in memory.

$$
\begin{aligned}
& \text { chainds }(d) \triangleq \circledast_{x \in \operatorname{dom}(d)} \text {. } \\
& x . \mathrm{up} \mapsto\{\mathrm{hdr}=d(x) . \text { uhdr; off }=d(x) . \text { uoff }\} * x . \text { loff } \mapsto(d(x) \text {.loff }) \\
& * \circledast_{i \in\{0 . . \operatorname{MAX}\}} \cdot x . f l a g s[i] \mapsto(d(x) \text {.flags }(i))
\end{aligned}
$$

Once a chan_addr object has been allocated, its up-address cannot be modified. To represent this in the definition, we write $x \mapsto v$ to indicate that $x$ is immutable - shorthand for $\exists f . x \stackrel{f}{\mapsto} v$. This is useful as immutable locations can be freely shared between threads: $x \mapsto v \Longrightarrow x \mapsto v * x \mapsto v$.

The interpretation function $I_{s}$ converts an abstract state Chain $(r s, d)$ into a datastructure:

$$
I_{s}(r)(\text { Chain }(r s, d)) \triangleq \operatorname{chainds}(d) * \text { chainres }(r s) * \text { unused }(r, r s, d)
$$

As chainds defines the concrete heap structure, it only requires a heap-map $d$. Conversely, chainres defines the pattern of splits, promises, and renunciations, and so only requires an abstract chain $r s$. Indeed, chainres is defined identically to $\S 6$.

The predicate unused, representing the 'library' of unused permissions, requires both $d$ and $r s$. This is because the position of a flag may move as a result of extension. As a result, wait may be passed a summary flag $x$ - before extension, the passed node would have been a leaf. The flag $x$ will be represented in $d$, but not represented in the chain $r s$, and thus unused requires both in order to keep track of permissions.

To define unused, the set $\mathrm{C}(r s, d)$ represents possible targets of wait; a permission is missing from the set of unused change permissions, $\mathrm{uC}(r s, d)$, only if it targets one of these 
nodes.

$$
\begin{aligned}
\mathrm{C}(r s, d) & \triangleq\left\{\langle y, i\rangle \mid r \in r s \wedge \text { finalleaf }_{d}(\langle y, i\rangle, r . l o c) \wedge \neg \text { finalleaf }_{d}(\langle d(y) . u h d r, d(y) . u o f f\rangle, r . l o c)\right\} \\
\mathrm{uC}(r s, d) & \triangleq\left\{(x, r) \mid r \in r s\left(\text { finalleaf }_{d}(x)\right) \cdot \mathcal{I} \wedge x \in \mathrm{C}(r s, d) \wedge \neg \exists y . r \in r s(y) . \mathcal{W}\right\} \\
\text { unused }(r, r s, d) & \triangleq\left(\circledast x \notin \mathrm{uS}(r s) .[\operatorname{send}(x)]_{1}^{r}\right) *\left(\circledast\left(x, r^{\prime}\right) \notin \mathrm{uC}(r s, d) .\left[\operatorname{change}\left(x, r^{\prime}\right)\right]_{1}^{r}\right)
\end{aligned}
$$

Predicate definitions. We can now define the send and recv predicates. These largely follow the definitions in $\S 6$ : the differences in underlying data-structures are abstracted by the interpretation function and transition relation.

$$
\begin{aligned}
\operatorname{oreg}(r, S) \triangleq & \operatorname{region}\left(S, T_{\mathrm{s}}, I_{\mathrm{s}}(r), r\right) \\
\operatorname{send}(x, P) \triangleq & \exists r_{1}, r_{2} \cdot r_{2} \stackrel{1 / 2}{\Longrightarrow} P *[\operatorname{send}(x)]_{1}^{r_{1}} *[\operatorname{mark}]_{-}^{r_{1}} * \\
& \operatorname{oreg}\left(r_{1},\left\{\text { Chain }(r s, d) \mid \operatorname{wf}(r s, d) \wedge r s(x)=\left(r_{2},,_{-}, 0,-\right)\right\}\right) \\
\operatorname{recv}(x, P) \triangleq & \exists r_{1}, r_{2} \cdot r_{2} \stackrel{1 / 2}{\longmapsto} P *\left[\operatorname{change}\left(x, r_{2}\right)\right]_{1}^{r_{1}} * \\
& \operatorname{oreg}\left(r_{1},\left\{\text { Chain }(r s, d) \mid \operatorname{wf}(r s, d) \wedge r_{2} \in r s\left(\text { finalleaf }_{d}(x)\right) \cdot \mathcal{I}\right\}\right) \\
x \prec y \triangleq & \exists r . \operatorname{oreg}\left(r,\left\{\text { Chain }(r s, d) \mid \operatorname{wf}(r s, d) \wedge\left(\text { finalleaf }_{d}(y), \text { finalleaf }_{d}(x)\right) \in \operatorname{ord}(r s)\right\}\right)
\end{aligned}
$$

We use $x$ to stand for $\langle x$.hdr, $x$.off $\rangle$ if $x$ is a chan_addr struct and we use [mark] $]_{-}^{r}$ as notation for $\exists \pi$. [mark $]_{\pi}^{r}$ to represent non-exclusive ownership of the mark action.

The stability of most of the predicates is obvious; however, the fact that finalleaf can change means we prove stability explicitly for recv.

Lemma 7.1. $\operatorname{recv}(x, P)$ is stable.

Proof. Assume the initial abstract state of the chain is Chain $(r s, d)$ and that ext takes the step $\left(x^{\prime},(r s, d),\left(r s^{\prime}, d^{\prime}\right)\right)$. The case where $x \neq x^{\prime}$ is trivial, so assume $x=x^{\prime}$. We now need to show $\operatorname{wf}\left(r s^{\prime}, d^{\prime}\right) \wedge r_{2} \in \mathrm{rs}^{\prime}\left(\right.$ finalleaf $\left._{d^{\prime}}(x)\right)$. I. Assume $a, b$ and $c$ are chain nodes as used in the definition of ext.

The requirement $\mathrm{wf}\left(r s^{\prime}, d^{\prime}\right)$ holds as a constraint on the transition relation. It remains to show the second clause. By the definition of ext, finalleaf ${ }_{d}(x)=a . l o c$ and $a \cdot \mathcal{I}=b . \mathcal{I}$. There are now two cases: either ext generates a new array, or it adds an element to the existing array. In the latter case, $d^{\prime}$ only changes by adding an element at a higher index in the array. Thus finalleaf ${ }_{d^{\prime}}(x)=b$.loc. In the former case, ext adds a new array which must also descend from $x$. If finalleaf $d^{\prime}(x) \neq b . l o c$, there must be leaf $z$ such that $b . l o c<_{d} z$, but the only new leaf $c$ is at the next index in the new array, meaning $c . l o c<_{d} b . l o c$. Thus the result follows by contradiction.

\subsection{Verifying wait, signal, extend}

Proving signal. A sketch-proof for signal is given in Fig. 27. The algorithm begins by setting the flag at the appropriate address (line 9). Abstractly the reasoning here is the same as when setting a flag in the non-summarising implementation ( $\S 6.3)$ so we omit it. The algorithm then climbs up the tree. If all the flags have been set in a given array, the summary flag is also set (line 9). Well-formedness allows summary nodes to be set if all their children are set. If a flag is discovered which is not set, or the loop climbs to the top of the tree, the algorithm exits.

The assignment on line 9 applies the transition relation step set or mark, depending on whether the node is a leaf or a summary. The following lemma ensures that the library of unused permissions is preserved after each such transition relation step. 


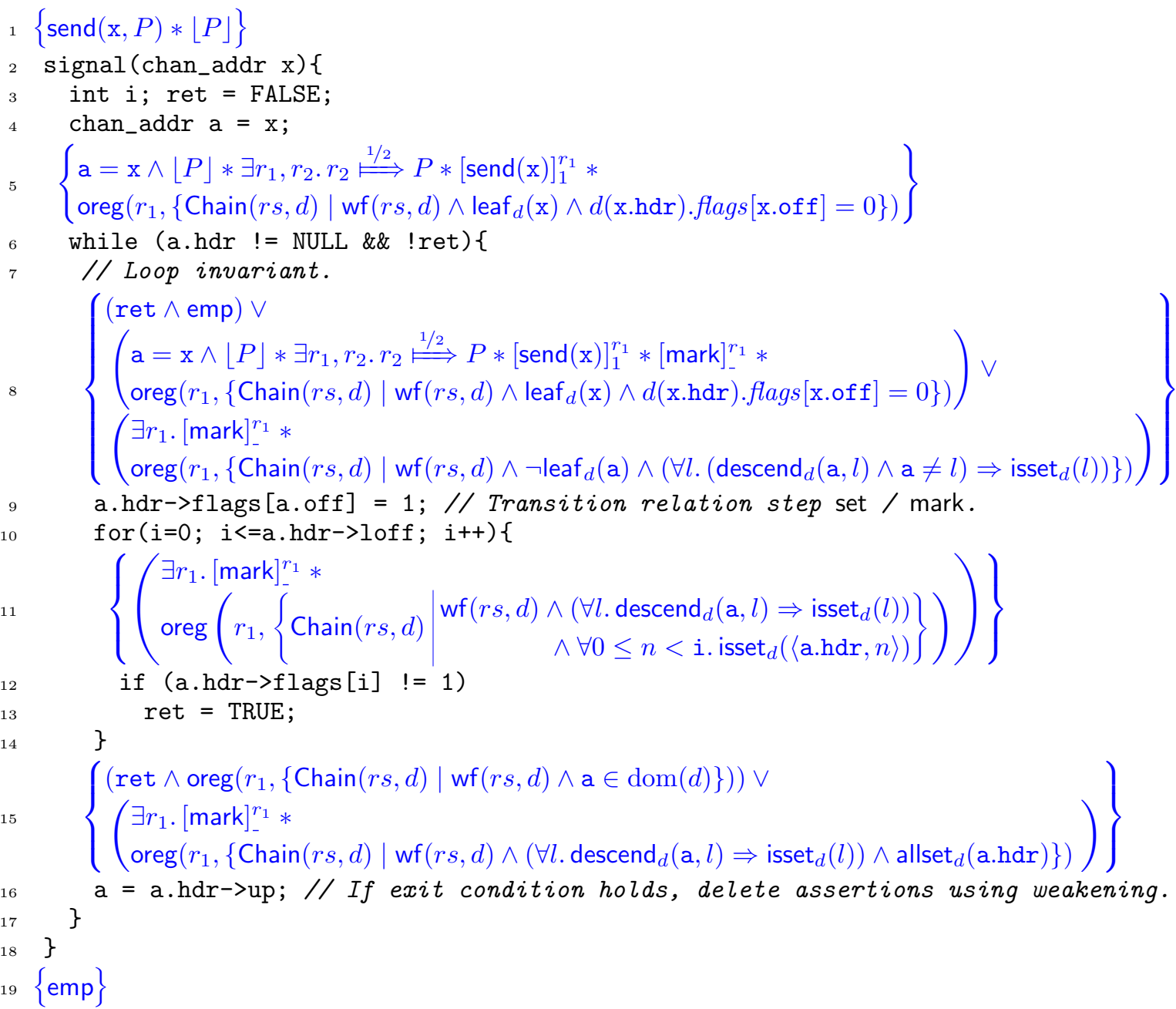

Fig. 27. Sketch-proof of signal with summarization. 


\section{LEMMA 7.2 .}

$$
\begin{aligned}
& \operatorname{set}_{s}\left(x,(r s, d),\left(r s^{\prime}, d^{\prime}\right)\right) \wedge \operatorname{unused}(r, r s, d) *[\operatorname{send}(x)]_{1}^{r} \Longrightarrow \operatorname{unused}\left(r, r s^{\prime}, d^{\prime}\right) \\
& \left((r s, d),\left(r s^{\prime}, d^{\prime}\right)\right) \in T_{s}(\text { mark }) \wedge \operatorname{unused}(r, r s, d) \Longrightarrow \operatorname{unused}\left(r, r s^{\prime}, d^{\prime}\right)
\end{aligned}
$$

Proof. Trivial from the definition of $T_{s}$ and unused.

Proving wait. A sketch-proof for wait is given in Fig. 28 / 29. This proof just deals with the part of the code establishing that all the flags in the chain have been set. In the proof, we use $\operatorname{last}_{d}(\mathrm{a})$ to stand for the last address in the array associated with a, i.e. $\langle$ a.hdr, $d($ a.hdr $)$.loff $\rangle$.

The loop starting at line 5 checks the flags in the current array. In line 8 the algorithm waits for the current node's flag. This may not be a leaf - it may be a summary node somewhere inside the tree. Once this passes, by the second clause of well-formedness (page 53) we can conclude that all the flags in the subsequence $r s_{3}$ have been set. Then the algorithm increments the offset - as a is not at the last offset for the array, there must exist an adjacent channel address at this position.

To prove this algorithm correct, we need several sub-lemmas. The first states that once the algorithm reaches the root of the tree, there are no locations in $d$ that are earlier according to $<_{d}$. This ensures that searching the tree covers all the preceding channels in the chain.

LEMma 7.3. $\quad \operatorname{wf}(d) \wedge d(a) . l o f f=o \wedge d(a) . u h d r=\mathrm{NULL} \Longrightarrow \neg \exists x . x<_{d}\langle a, o\rangle$

Proof. Assume such an $x$ exists. Then by the definition of $<_{d}$, there must exist an address $y$ such that $x$ and $\langle a, o\rangle$ are both descended from $y$. As the $u h d r$ field is NULL, the only possibility is that both addresses are in the object at $a$. By the definition of $<_{d}, x$ must be further right in the flag array, but $o$ is the right-most address. This contradicts the assumption and completes the proof.

The second lemma states that examining the elements reachable through the heap map suffices to show that the corresponding elements in the abstract chain have been set. This lemma justifies our splitting of the invariant into a separate heap map and abstract chain structure.

LEMma 7.4. $\quad w f(r s, d) \wedge z \in \operatorname{dom}(r s) \wedge \operatorname{descend}_{d}(x, z) \wedge \operatorname{leaf}_{d}(z) \wedge$

$$
\begin{aligned}
& \left(\forall l . l<<_{d}^{\mathbb{N}} x \wedge \operatorname{leaf}_{d}(l) \Rightarrow \operatorname{isset}_{d}(l)\right) \\
& \quad \Longrightarrow \quad \exists r s_{1}, r s_{2} . r s=r s_{1} \cdot r s(z) \cdot r s_{2} \wedge \operatorname{ctrue}\left(r s(z) \cdot r s_{2}\right)
\end{aligned}
$$

Proof. As $z \in \operatorname{dom}(r s)$, we can easily divide up $r s$ into $r s_{1} \cdot r s(z) \cdot r s_{2}$. Now pick an arbitrary element $y$ in $\operatorname{dom}\left(r s(z) \cdot r s_{2}\right)$ and suppose that $r s(y)$. flg is not set. By wellformedness, it must be true that $y<_{d}^{\mathbb{}} z$. Now we show that $y<_{d}^{\mathbb{N}} x$. The contrary, $x<_{d} y$, would imply that $z<_{d} y$, contradicting our assumption. Therefore by the premise the associated flag must be set. However, well-formedness requires that flags are mirrored in $r s$ and $d$, contradicting our assumption and completing the proof.

The final lemma shows that shifting left from the current maximal node reaches a node earlier in the order. Note that the existence of the new node is shown on left of the implication because this lemma applied in a negative position in the proof.

LEMma 7.5. $0 \leq d(a)$. off $<d(a) . \operatorname{loff} \wedge \mathrm{wf}(d) \wedge a \bullet_{\text {off }}(\bullet+1)<_{d} k<_{d} b \Longrightarrow a<_{d}^{\mathbb{k}} k<_{d} b$

Proof. The result follows from the structure of the heap map and the definition of $<_{d}$.

Proving extend. A sketch-proof of extend is given in Fig. 30. There are two cases for extending the chain: either the node is the last element in the current array and there is 


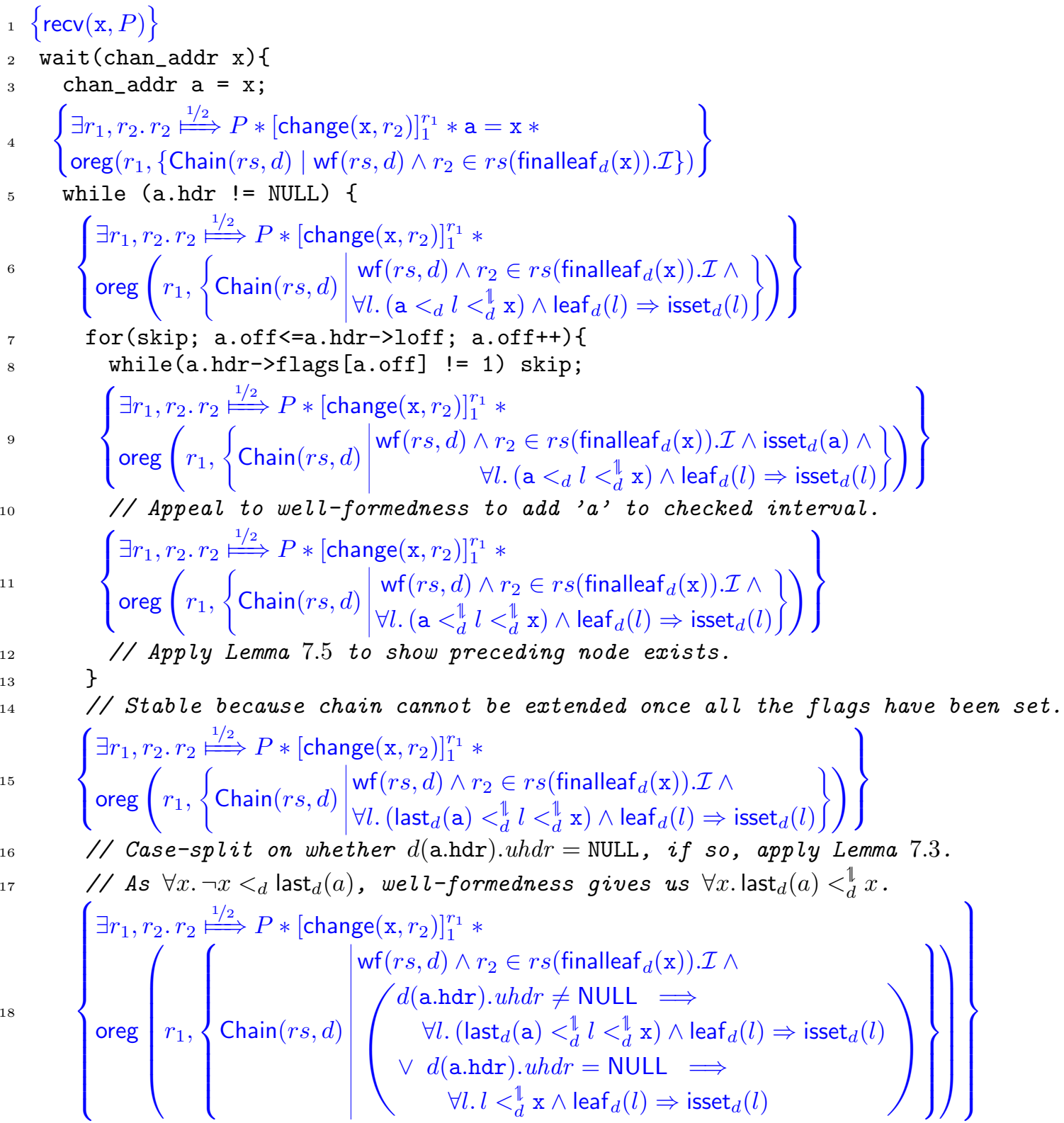

Fig. 28. Sketch-proof of wait with summarization (completed in Fig. 29). 
$\mathrm{a}=\mathrm{a} \cdot \mathrm{hdr}->\mathrm{up}$

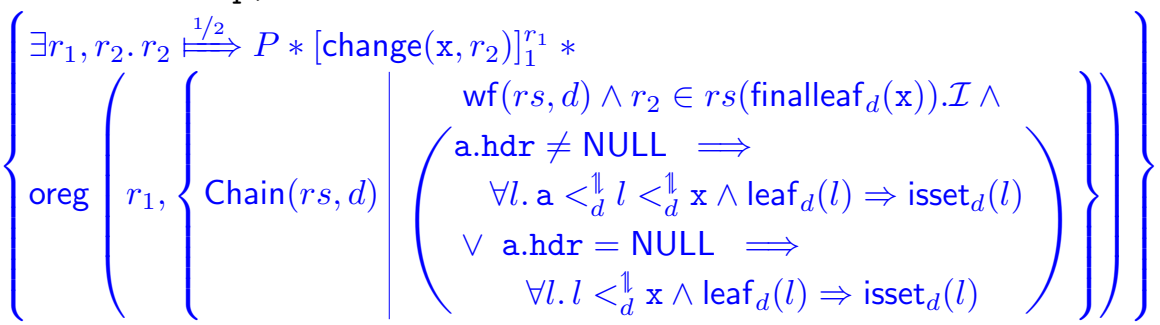

a.off++; // Apply Lemma 7.5 to non-NULL case.

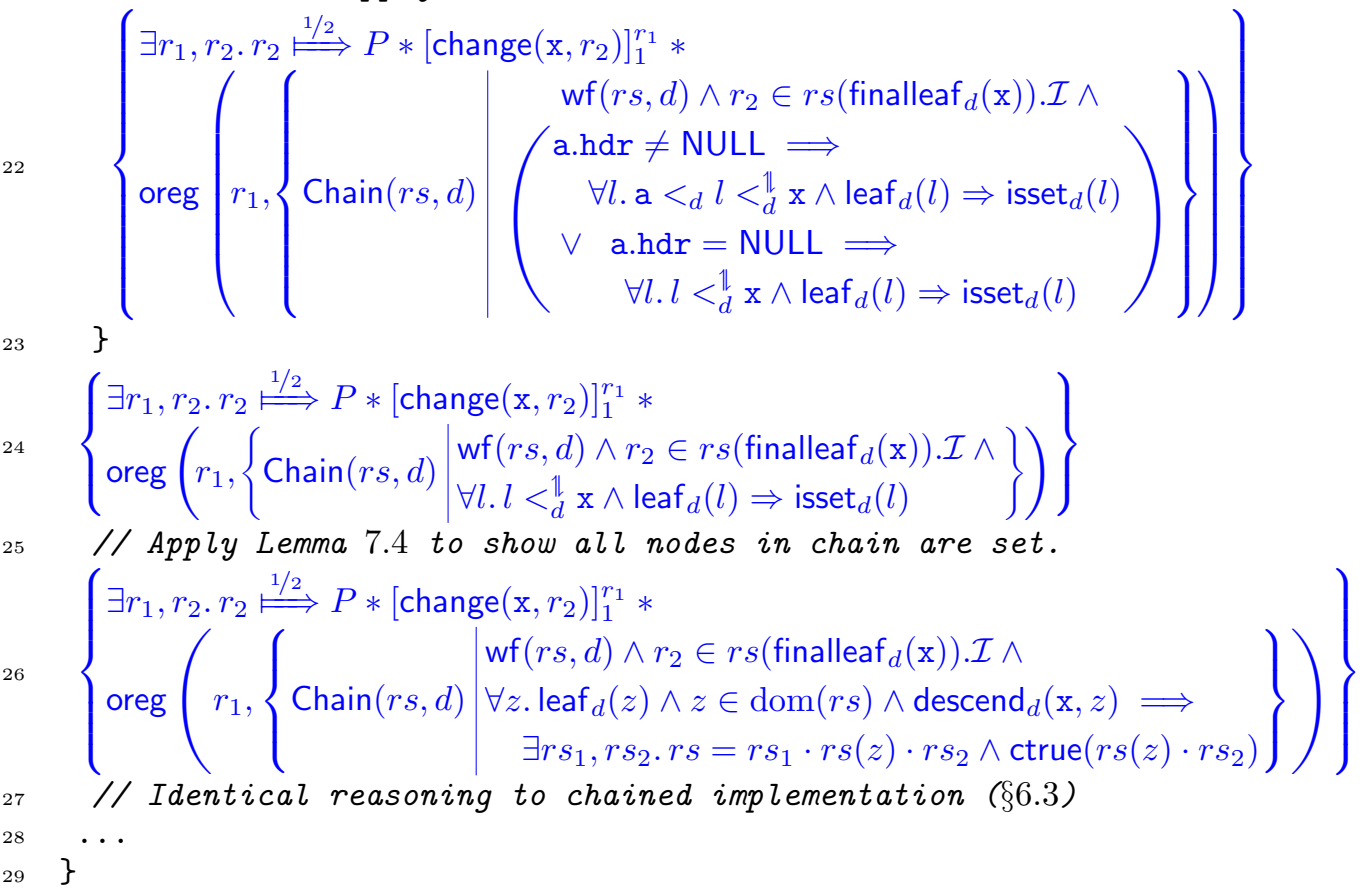

Fig. 29. Sketch-proof of wait with summarization (continued from Fig. 28).

space to add an extra node; or there is no space and the algorithm allocates a fresh array. This choice is made by the conditional in line 6 .

The proof needs the following lemmas to show that the unused predicate representing unused permissions is preserved by extending the chain.

LEMMA 7.6.

$$
\begin{aligned}
\left(\begin{array}{l}
\operatorname{ext}_{s}\left(\langle x, i\rangle,(r s, d),\left(r s^{\prime}, d^{\prime}\right)\right) \wedge \operatorname{dom}\left(d^{\prime}\right)=\operatorname{dom}(d) \wedge r^{\prime} \notin r s \wedge \\
r s^{\prime}(\langle x, i+1\rangle) \cdot \mathcal{I}=\left\{r^{\prime}\right\} \wedge \operatorname{wf}(r s, d) \wedge \operatorname{wf}\left(r s^{\prime}, d^{\prime}\right) \wedge \operatorname{unused}(r, r s, d)
\end{array}\right) \Longrightarrow \\
\text { unused }\left(r, r s^{\prime}, d^{\prime}\right) *[\operatorname{send}(\langle x, i+1\rangle)]_{1}^{r} *\left[\operatorname{change}\left(\langle x, i+1\rangle, r^{\prime}\right)\right]_{1}^{r}
\end{aligned}
$$

ProOF. Begin by observing that, by the definition of $\operatorname{ext}_{s},\langle x, i+1\rangle \notin r s$, and thus that: $\operatorname{unused}(r, r s, d) \Longrightarrow[\operatorname{send}(\langle x, i+1\rangle)]_{1}^{r} *\left[\operatorname{change}\left(\langle x, i+1\rangle, r^{\prime}\right)\right]_{1}^{r} *$ true

As $\langle x, i+1\rangle \in r s^{\prime}$, it holds immediately that $\langle x, i+1\rangle \in \mathrm{uS}\left(r s^{\prime}\right)$. As $\langle x, i+1\rangle$ is a leaf, finalleaf $_{d^{\prime}}(\langle x, i+1\rangle)=\langle x, i+1\rangle$. As it is not the first leaf in the array $x$, it cannot have a 


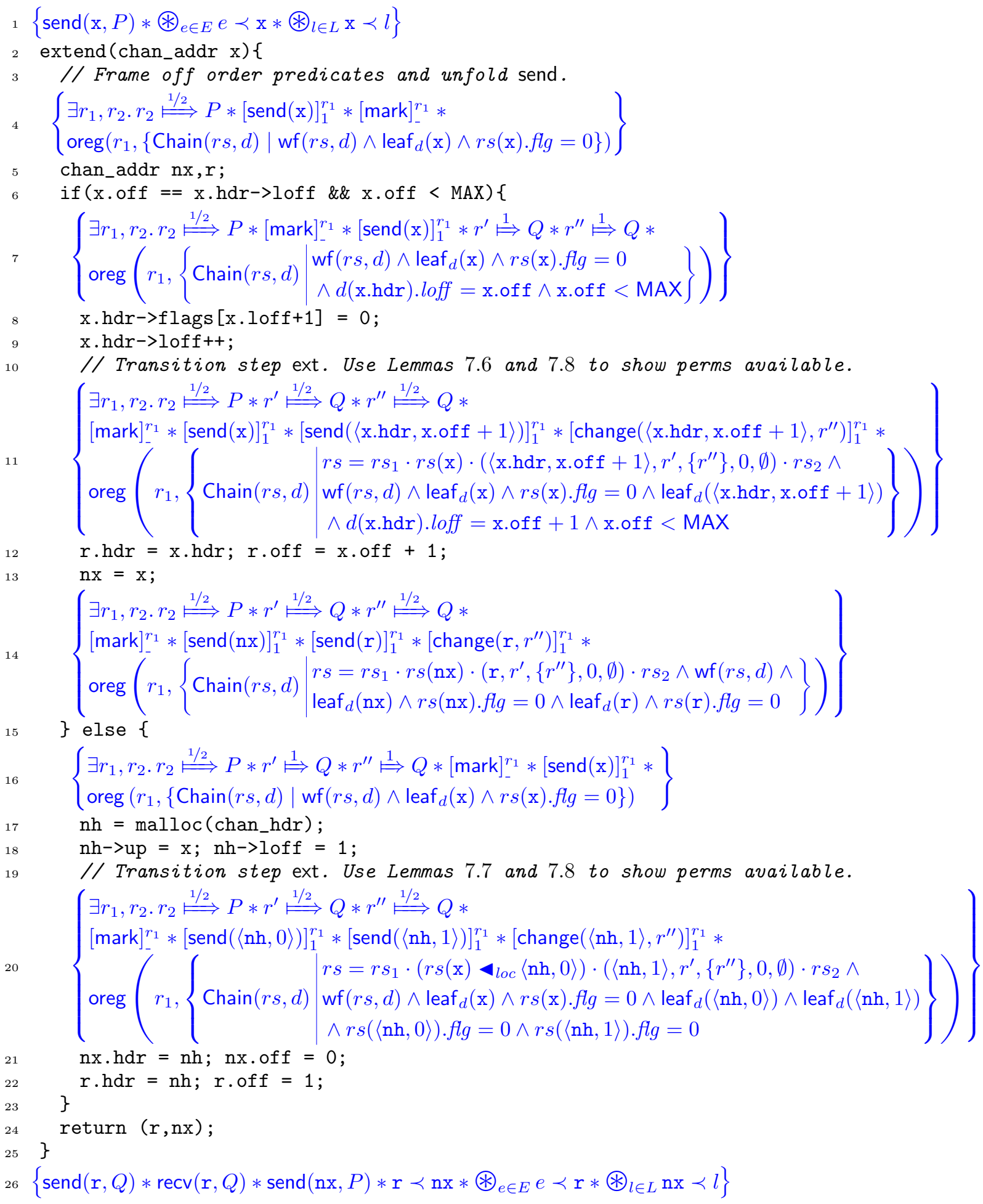

Fig. 30. Sketch-proof of extend with summarization. 
finalleaf parent, meaning it must be in $\mathrm{C}\left(r s^{\prime}, d^{\prime}\right)$. Thus $\langle x, i+1\rangle \in \mathrm{uC}\left(r s^{\prime}, d^{\prime}\right)$. This suffices to show that $[\operatorname{send}(\langle x, i+1\rangle)]$ and $\left[\right.$ change $\left.\left(\langle x, i+1\rangle, r^{\prime}\right)\right]$ can be safely removed from unused.

LEMMA 7.7.

$$
\begin{gathered}
\left(\begin{array}{l}
\operatorname{ext}_{s}\left(\langle x, i\rangle,(r s, d),\left(r s^{\prime}, d^{\prime}\right)\right) \wedge \operatorname{dom}\left(d^{\prime}\right)=\operatorname{dom}(d) \uplus\{l\} \wedge r^{\prime} \notin r s \wedge \\
r s^{\prime}(\langle l, 1\rangle) \cdot \mathcal{I}=\left\{r^{\prime}\right\} \wedge \operatorname{wf}(r s, d) \wedge \operatorname{wf}\left(r s^{\prime}, d^{\prime}\right) \wedge \operatorname{unused}(r, r s, d) *[\operatorname{send}(\langle x, i\rangle)]_{1}^{r}
\end{array}\right) \Longrightarrow \\
\quad \text { unused }\left(r, r s^{\prime}, d^{\prime}\right) *[\operatorname{send}(\langle l, 0\rangle)]_{1}^{r} *[\operatorname{send}(\langle l, 1\rangle)]_{1}^{r} *\left[\operatorname{change}\left(\langle l, 1\rangle, r^{\prime}\right)\right]_{1}^{r}
\end{gathered}
$$

Proof. By the structure of ext, $\langle l, 0\rangle$ and $\langle l, 1\rangle$ are not in $r s$, but are in $r s^{\prime}$. The ability to retrieve $[\operatorname{send}(\langle l, 0\rangle)]_{1}^{r} *[\operatorname{send}(\langle l, 1\rangle)]_{1}^{r}$ follows immediately. As $\langle l, 0\rangle$ is leftmost in the array $l$, its parent $\langle x, i\rangle$ is the maximal final-leaf in $\mathrm{C}\left(r s^{\prime}, d^{\prime}\right)$. However, $\langle l, 1\rangle$ is not leftmost, and thus is in $\mathrm{C}\left(r s^{\prime}, d^{\prime}\right)$. By the same argument used in the previous lemma, [change $\left.\left(\langle l, 1\rangle, r^{\prime}\right)\right]_{1}^{r}$ can be removed from the unused.

Lemma 7.8. $\operatorname{ext}\left(x,(r s, d),\left(r s^{\prime}, d^{\prime}\right)\right) \wedge \operatorname{wf}(r s, d) \wedge \operatorname{wf}\left(r s^{\prime}, d^{\prime}\right) \Longrightarrow \mathrm{uC}(r s, d) \subseteq \mathrm{uC}\left(r s^{\prime}, d^{\prime}\right)$

Proof. There are two cases for extension: in-place extension in the array, or creation of a new array. In the former case, finalleaf is preserved for existing nodes because the only new node is less than all existing nodes in the array. In the latter case, the parent of the new array is a finalleaf to the new array, and all other finalleaf relationships are preserved.

Now pick a pair $(x, i) \in \mathrm{uC}(r s, d)$, the set of used send permissions. Extending the chain can't stop $x$ from satisfying finalleaf or make any node higher than $x$ satisfy finalleaf. Therefore $x \in \mathrm{C}\left(r s^{\prime}, d^{\prime}\right)$ after extension. The only alteration to renounced sets $\mathcal{W}$ in $r s^{\prime}$ is to add a new empty set. Thus $\neg \exists y . r \in r s^{\prime}(y)$. W. Finally, both cases of extension preserve the promise sets $\mathcal{I}$, ensuring that $r \in r s^{\prime}\left(\right.$ finalleaf $\left._{d^{\prime}}(x)\right)$.I .

\subsection{Verifying splitting and renunciation axioms}

The splitting and renunciation axioms do not depend on the underlying data-structure representation, and therefore are largely identical to the ones given in $\S 6.4$. The main difference is the new definition of unused. The renunciation case is straightforward, but for splitting we need to show that we can pull the appropriate change permissions out of the 'library' predicate unused. This is captured by the following lemma:

\section{LEMMA 7.9.}

$$
\begin{aligned}
& \operatorname{unused}\left(r_{1}, r s, d\right) *\left[\operatorname{change}\left(x, r_{2}\right)\right]_{1}^{r_{1}} \wedge \operatorname{split}\left(x,(r s, d),\left(r s^{\prime}, d^{\prime}\right)\right) \wedge \\
& r s\left(\text { finalleaf }_{d}(x)\right)=\left(r, \mathcal{I} \uplus\left\{r_{2}\right\}, f, \mathcal{W}\right) \wedge r s^{\prime}=r s \triangleleft_{\text {finalleaf }_{d^{\prime}}(x)} \boldsymbol{\cup}_{\mathcal{I}}\left(\left(\bullet \backslash\left\{r_{2}\right\}\right) \uplus\left\{r_{3}, r_{4}\right\}\right) \\
& \Longrightarrow \text { unused }\left(r_{1}, r s^{\prime}, d^{\prime}\right) *\left[\operatorname{change}\left(x, r_{3}\right)\right]_{1}^{r_{1}} *\left[\operatorname{change}^{\prime}\left(x, r_{4}\right)\right]_{1}^{r_{1}}
\end{aligned}
$$

Proof. By the definition of split, $d=d^{\prime}$, and element locations in $r s$ are unchanged in $r s^{\prime}$. Thus it holds that $\mathrm{C}(r s, d)=\mathrm{C}\left(r s^{\prime}, d^{\prime}\right)$ and finalleaf $f_{d}(x)=$ finalleaf $_{d^{\prime}}(x)$. From the definition of $\mathrm{uC}$ the available change permissions are controlled by the set $r s\left(\right.$ finalleaf $\left._{d}(x)\right)$. $\mathcal{I}$. This set is correctly updated by the transition, which completes the proof.

\section{COMPARISON TO CONFERENCE PAPER}

This paper substantially expands and revises the proofs of correctness given in our conference paper [Dodds et al. 2011]. All the proofs have been restructured, and the proof of the summarising implementation $(\S 7)$ is entirely new. This paper also fixes a subtle logical error which rendered some of the reasoning in our conference paper unsound. In this section, we describe how this problem arose, and how we have fixed it.

Our specifications rely crucially on higher-order quantification to abstract over the resources transferred through channels. To support this, in [Dodds et al. 2011] we extended the 
original concurrent abstract predicates logic [Dinsdale-Young et al. 2010] with higher-order assertions and quantification.

In concurrent abstract predicates, resources describe not only the current state of shared regions but also the protocols that govern these shared regions. In the case of higher-order shared resources, these protocols are themselves expressed in terms of assertion variables that might be instantiated with shared resources. Support for such higher-order shared resources thus require a semantic domain of protocols that include assertions over (among other things) protocols. This results in a circularity and the resulting equation (protocol $\cong$ $\mathcal{P}(\ldots \times$ protocol $))$ has no solution in set-theory, by a simple cardinality argument.

The logic and model presented in [Dodds et al. 2011] broke this circularity by ignoring protocol assertions when interpreting protocols. As a consequence, many of the properties we relied on when reasoning about the higher-order resources box $(i, P, \pi)$ and fut $(i, P)$ are unsound. (In that paper, fut played a similar role to recv in this paper, while box was used in verifying the splitting axiom.) For instance, fut $(i, P)$ is generally not stable when $P$ is instantiated with an assertion that includes a protocol assertion, because fut $(i, P)$ asserts the existence of a shared region whose protocol is defined in terms of $P$. A more detailed discussion of this class of problem is given in [Svendsen et al. 2013].

The program logic itself presented in [Dodds et al. 2011] still appears sound. However, many steps in the proofs of programs depend on unsound auxiliary entailment steps. These steps are common in separation logic proofs, but in most earlier work entailments generally capture comparatively simple properties. We failed to appreciate how deeply the proofs in [Dodds et al. 2011] relied on very subtle entailments between shared regions that were broken in our modified model. The problem came to light a year later when Svendsen attempted to use our logic to verify the Joins library [Svendsen et al. 2013]. Resolving this kind of problem motivated the development of iCAP, which is the proof technique we use in this paper.

iCAP uses step-indexing to stratify the construction of the semantic domain of protocols. The resulting logic does support higher-order shared resources, but requires $\triangleright$ operators to ensure that protocols are properly stratified. Thus the problematic circularity in [Dodds et al. 2011] is appropriately resolved in the rules of the logic. At the level of human process, we have been much more meticulous in this paper in identifying and checking entailment steps used in program proofs.

Acknowledgements. Thanks to the anonymous referees, Richard Bornat, Matko Botinčan, Thomas Dinsdale-Young, Philippa Gardner, Ralf Jung, Robbert Krebbers, Neel Krishnaswami, Daiva Naudžiūniené, Viktor Vafeiadis and John Wickerson.

\section{REFERENCES}

Christian J. Bell, Andrew Appel, and David Walker. 2009. Concurrent Separation Logic for Pipelined Parallelization. In $S A S$.

Emery D. Berger, Ting Yang, Tongping Liu, and Gene Novark. 2010. Grace: Safe Multithreaded Programming for $\mathrm{C} / \mathrm{C}++$. In OOPSLA.

Lars Birkedal, Rasmus Ejlers Møgelberg, Jan Schwinghammer, and Kristian Støvring. 2012. First steps in synthetic guarded domain theory: step-indexing in the topos of trees. Logical Methods in Computer Science 8, 4 (2012).

Robert L. Bocchino, Jr., Vikram S. Adve, Danny Dig, Sarita V. Adve, Stephen Heumann, Rakesh Komuravelli, Jeffrey Overbey, Patrick Simmons, Hyojin Sung, and Mohsen Vakilian. 2009. A type and effect system for deterministic parallel Java. In OOPSLA '09. ACM, 97-116.

Richard Bornat, Cristiano Calcagno, Peter O'Hearn, and Matthew Parkinson. 2005. Permission accounting in separation logic. In POPL. 259-270.

M. Botinčan, M. Dodds, and S. Jagannathan. 2013. Resource-Sensitive Synchronization Inference by Abduction. In ACM Trans. on Programming Languages and Systems.

P. da Rocha Pinto, T. Dinsdale-Young, M. Dodds, P. Gardner, and M. Wheelhouse. 2011. A Simple Abstraction for Complex Concurrent Indexes. In OOPSLA. 
P. da Rocha Pinto, T. Dinsdale-Young, and P. Gardner. 2014. TaDA: A Logic for Time and Data Abstraction. In ECOOP. To appear.

Thomas Dinsdale-Young, Mike Dodds, Philippa Gardner, Matthew J Parkinson, and Viktor Vafeiadis. 2010. Concurrent Abstract Predicates. In ECOOP.

Mike Dodds, Xinyu Feng, Matthew J. Parkinson, and Viktor Vafeiadis. 2009. Deny-Guarantee Reasoning. In ESOP.

M. Dodds, S. Jagannathan, and M. J. Parkinson. 2011. Modular Reasoning for Deterministic Parallelism. In $P O P L$.

Xinyu Feng, Rodrigo Ferreira, and Zhong Shao. 2007. On the Relationship between Concurrent Separation Logic and Assume-Guarantee Reasoning. In ESOP.

Alexey Gotsman, Josh Berdine, Byron Cook, Noam Rinetzky, and Mooly Sagiv. 2007. Local Reasoning for Storable Locks and Threads. In APLAS.

Christian Haack, Marieke Huisman, and Clément Hurlin. 2008. Reasoning about Java's Reentrant Locks. In APLAS. $171-187$.

C. A. R. Hoare and Peter W. O'Hearn. 2008. Separation Logic Semantics for Communicating Processes. ENTCS 212 (2008), 3-25.

Aquinas Hobor, Andrew W. Appel, and Francesco Zappa Nardelli. 2008. Oracle Semantics for Concurrent Separation Logic. In ESOP.

Bart Jacobs and Frank Piessens. 2009. Modular Full Functional Specification and Verification of LockFree Data Structures. Technical Report CW 551. Katholieke Universiteit Leuven, Dept. of Computer Science.

Cliff B. Jones. 1983. Tentative steps toward a development method for interfering programs. TOPLAS 5, 4 (1983), 596-619.

Neel R. Krishnaswami, Lars Birkedal, and Jonathan Aldrich. 2010. Verifying event-driven programs using ramified frame properties. In TLDI.

K. R. M. Leino, P. Müller, and J. Smans. 2010. Deadlock-free Channels and Locks. In ESOP.

A. Navabi, X. Zhang, and S. Jagannathan. 2008. Quasi-static Scheduling for Safe Futures. In PPoPP. ACM, $23-32$.

Peter W. O'Hearn. 2007. Resources, Concurrency and Local Reasoning. TCS (2007).

M. J. Parkinson and G. M. Bierman. 2005. Separation logic and abstraction. In POPL. 247-258.

Martin C. Rinard and Monica S. Lam. 1992. Semantic Foundations of Jade. In POPL. ACM, 105-118.

Kasper Svendsen and Lars Birkedal. 2014a. Impredicative Concurrent Abstract Predicates. In ESOP.

Kasper Svendsen and Lars Birkedal. 2014b. Impredicative Concurrent Abstract Predicates. Technical Report. Aarhus University. https://bitbucket.org/logsem/public/src/master/icap/esop2014-tr.pdf

Kasper Svendsen, Lars Birkedal, and Matthew J. Parkinson. 2013. Joins: A Case Study in Modular Specification of a Concurrent Reentrant Higher-Order Library. In ECOOP.

Aaron Turon, Derek Dreyer, and Lars Birkedal. 2013. Unifying refinement and hoare-style reasoning in a logic for higher-order concurrency. In ICFP.

Viktor Vafeiadis. 2007. Modular Fine-Grained Concurrency Verification. Ph.D. Dissertation. University of Cambridge.

Viktor Vafeiadis and Matthew J Parkinson. 2007. A marriage of Rely/Guarantee and Separation Logic. In CONCUR. 256-271.

Jules Villard, Étienne Lozes, and Cristiano Calcagno. 2010. Tracking Heaps That Hop with Heap-Hop. In TACAS. 275-279.

A. Welc, S. Jagannathan, and A. Hosking. 2005. Safe Futures for Java. In OOPSLA. 439-435.

John Wickerson, Mike Dodds, and Matthew Parkinson. 2010. Explicit Stabilisation for Modular RelyGuarantee Reasoning. In ESOP. 


\section{A. SAVED PROPOSITIONS}

Lemma A.1. Property (3) of saved propositions implies property (4).

ProOF.

$$
\begin{aligned}
& r \stackrel{\pi_{1}}{\Longrightarrow} P * r \stackrel{\pi_{2}}{\Longrightarrow} Q *(X * \triangleright(Q * Y)) *(P * Z) \\
& \text { Property }(3), \text { frame off saved propositions. } \\
& \Longrightarrow \quad(X * \triangleright(Q * Y)) *(P * Z) *(\triangleright Q \Rightarrow \triangleright P) \\
& \quad \text { Apply } \mathrm{SMONO}, \triangleright(P * Q) \Longrightarrow(\triangleright P * \triangleright Q), \text { and }(P \Rightarrow Q) \Longrightarrow(P * Q) . \\
& \Longrightarrow \quad(X * \triangleright(Q * Y)) *(\triangleright P * \triangleright Z) *(\triangleright Q * \triangleright P) \\
& \text { Merge } * . \\
& \Longrightarrow \quad(X * \triangleright(Q * Y)) *(\triangleright Q * \triangleright Z) \\
& \text { Add frame } \triangleright Y \text { to } * \text {, rearrange } \triangleright . \\
& \Longrightarrow \quad(X * \triangleright(Q * Y)) *(\triangleright(Q * Y) * \triangleright(Z * Y)) \\
& \text { Merge } *, \text { frame saved propositions back on. } \\
& \Longrightarrow \quad r \stackrel{\pi_{1}}{\Longrightarrow} P * r \stackrel{\pi_{2}}{\Longrightarrow} Q *(X * \triangleright(Z * Y))
\end{aligned}
$$

\section{A.1. Encoding in iCAP}

A saved proposition $r \stackrel{\pi}{\Rightarrow} P$ is encoded as a normal iCAP predicate with a structure guaranteeing the properties we want. Intuitively, this predicate consists of a shared region with identifier $r$, with the proposition $P$ encoded into its transition relation. Linearity comes from a permission with fractional argument $\pi$.

More formally, we assume two transition-system states $\{1$ st, 2nd $\}$ and a single token tok, and invariant map $I_{\text {prp }}$ and transition relation $T_{\text {prp }}$ defined as follows:

$$
\begin{aligned}
I_{\text {prp }}(Q)(1 \mathrm{st}) & \triangleq \mathrm{emp} \\
I_{\mathrm{prp}}(Q)(2 \mathrm{nd}) & \triangleq Q \\
T_{\mathrm{prp}}(\text { tok }) & \triangleq\{(1 \text { st }, 2 \text { nd })\}
\end{aligned}
$$

We then define the saved proposition $r \stackrel{\pi}{\Leftrightarrow} Q$ as follows:

$$
r \stackrel{\pi}{\Longrightarrow} Q \triangleq \operatorname{region}\left(\{1 \mathrm{st}, 2 \mathrm{nd}\}, T_{\mathrm{prp}}, I_{\mathrm{prp}}(Q), r\right) *[\text { tok }]_{r}^{\pi}
$$

The fact that the representation transition state 1 st is emp means that we are not obliged to supply $P$ when creating the saved proposition. The second state encodes the value of the saved proposition.

The linearity property (property 2 ) holds trivially from the linearity of permissions. Two saved propositions with arguments $\pi_{1}$ and $\pi_{2}$ must contain tok permissions with fractional $\operatorname{arguments} \pi_{1}$ and $\pi_{2}$. Combining these gives the required result.

For the unification property (property 3 ) we need to reason more deeply about the iCAP model. The following facts about regions and invariant maps hold in iCAP - for proofs see [Svendsen and Birkedal 2014b].

$$
\begin{aligned}
& \operatorname{region}(S, T, I, r) * \operatorname{region}\left(S^{\prime}, T^{\prime}, J, r\right) \quad \Longrightarrow \quad(\triangleright I(s) \Rightarrow \triangleright J(s)) \\
& \operatorname{region}(S, T, I, r) * \operatorname{region}\left(S^{\prime}, T^{\prime}, J, r\right) \quad \Longrightarrow \quad(\triangleright I(s) * \triangleright J(s))
\end{aligned}
$$

The later modality, $\triangleright$, is needed in these properties because we are reasoning about the contents of a shared region - albeit one that will not contain any resource. We can then prove the unification property as follows: 
Proof (Property 3).

$$
\begin{aligned}
& r \stackrel{\pi_{1}}{\Longleftrightarrow} P * r \stackrel{\pi_{2}}{\Longleftrightarrow} Q \\
& \Rightarrow r \stackrel{\pi_{1}}{\Longleftrightarrow} P * \text { region }\left(\{1 \mathrm{st}, 2 \mathrm{nd}\}, T_{\mathrm{prp}}, I_{\mathrm{prp}}(P), r\right) \quad \text { region is duplicable. } \\
& * r \stackrel{\pi_{2}}{\Longrightarrow} Q * \operatorname{region}\left(\{1 \mathrm{st}, 2 \mathrm{nd}\}, T_{\mathrm{prp}}, I_{\mathrm{prp}}(Q), r\right) \\
& \Rightarrow r \stackrel{\pi_{1}}{\Longleftrightarrow} P * r \stackrel{\pi_{2}}{\Longleftrightarrow} Q *\left(\triangleright\left(I_{\mathrm{prp}}(P)(2 \mathrm{nd})\right) \Rightarrow \triangleright\left(I_{\mathrm{prp}}(Q)(2 \mathrm{nd})\right)\right) \text { Property } 5 \text {. } \\
& \Rightarrow r \stackrel{\pi_{1}}{\Longleftrightarrow} P * r \stackrel{\pi_{2}}{\Longleftrightarrow} Q *(\triangleright P \Rightarrow \triangleright Q) \quad \text { defn of } I_{\text {prp }}
\end{aligned}
$$

For unification inside separating implication we reason as follows:

Proof (Property 4). Using property 6 and the same proof technique as above, we can derive a slightly different version of the unification property:

$$
r \stackrel{\pi_{1}}{\Longleftrightarrow} P * r \stackrel{\pi_{2}}{\Longleftrightarrow} Q \quad \Longrightarrow \quad(\triangleright P) *(\triangleright Q)
$$

The proof of Property (4) then goes as follows:

$$
\begin{aligned}
& r \stackrel{\pi_{1}}{\Longleftrightarrow} P * r \stackrel{\pi_{2}}{\Longrightarrow} Q *(X * \triangleright(Q * Y)) *(P * Z) \\
& \text { SMono } \\
& \Rightarrow r \stackrel{\pi_{1}}{\Longleftrightarrow} P * r \stackrel{\pi_{2}}{\Longleftrightarrow} Q *(X * \triangleright(Q * Y)) * \triangleright(P * Z) \\
& \text { LBin, assume } \triangleright \text { distributes over } * \\
& \Rightarrow r \stackrel{\pi_{1}}{\Longleftrightarrow} P * r \stackrel{\pi_{2}}{\Longleftrightarrow} Q *(X *(\triangleright Q * \triangleright Y)) *((\triangleright P) * \triangleright Z) \\
& \text { Assume property } 7 \\
& \Rightarrow r \stackrel{\pi_{1}}{\Longleftrightarrow} P * r \stackrel{\pi_{2}}{\Longleftrightarrow} Q *(X *(\triangleright Q * \triangleright Y)) *((\triangleright P) * \triangleright Z) *((\triangleright Q) *(\triangleright P)) \\
& \text { Transitivity of } * \\
& \Rightarrow r \stackrel{\pi_{1}}{\Longleftrightarrow} P * r \stackrel{\pi_{2}}{\Longleftrightarrow} Q *(X *(\triangleright Q * \triangleright Y)) *((\triangleright Q) * \triangleright Z) \\
& \text { Framing of } * \\
& \Rightarrow r \stackrel{\pi_{1}}{\Longleftrightarrow} P * r \stackrel{\pi_{2}}{\Longleftrightarrow} Q *(X *(\triangleright Q * \triangleright Y)) *((\triangleright Q) *(\triangleright Y)) *((\triangleright Z) *(\triangleright Y)) \\
& \text { Transitivity of } * \\
& \Rightarrow r \stackrel{\pi_{1}}{\Longleftrightarrow} P * r \stackrel{\pi_{2}}{\Longleftrightarrow} Q *(X *((\triangleright Z) *(\triangleright Y)))
\end{aligned}
$$

\section{B. PROOFS FOR OUT-OF-ORDER SIGNALLING}

This appendix gives proofs for some of the lemmas stated in $\S 6$.

LEMMA 6.1.

$$
\operatorname{resource}(\mathcal{I}, \emptyset) \sqsubseteq \underset{i \in \mathcal{I}}{\circledast} . \exists Q: \text { Prop. } i \stackrel{1 / 2}{\Longleftrightarrow} Q(i) *\lceil\triangleright Q(i)\rceil
$$


Proof.

$$
\begin{aligned}
& \mathcal{W}=\emptyset \wedge \exists Q: \mathcal{I} \rightarrow \text { Prop, } R: \mathcal{W} \rightarrow \text { Prop. } \\
& \circledast_{i \in \mathcal{I}} \cdot i \stackrel{1 / 2}{\longmapsto} Q(i) * \circledast_{w \in \mathcal{W}} \cdot w \stackrel{1 / 2}{\longmapsto} R(w) \\
& *\left\lfloor\left(\triangleright \circledast_{w \in \mathcal{W}} \cdot R(w)\right) * \triangleright \circledast_{i \in \mathcal{I}} \cdot\lceil Q(i)\rceil\right\rfloor \\
& \text { Simplify using } \mathcal{W}=\emptyset \text {, weakening. } \\
& \sqsubseteq \quad \exists Q: \mathcal{I} \rightarrow \text { Prop. } \circledast_{i \in \mathcal{I}} \cdot i \stackrel{1 / 2}{\Longleftrightarrow} Q(i) *\left\lfloor\triangleright \circledast_{i \in \mathcal{I}} \cdot Q(i)\right\rfloor \\
& \text { Switch from }\lfloor-\rfloor \text { to }\lceil-\rceil \text {, pull out } \circledast \text {. } \\
& \sqsubseteq \quad \exists Q: \mathcal{I} \rightarrow \text { Prop. } \circledast_{i \in \mathcal{I}} \cdot i \stackrel{1 / 2}{\Longleftrightarrow} Q(i) * \circledast_{i \in \mathcal{I}} \cdot\lceil\triangleright Q(i)\rceil \\
& \text { Push in the existential. } \\
& \sqsubseteq \circledast_{i \in \mathcal{I}} \cdot \exists Q \text { : Prop. } i \stackrel{1 / 2}{\Longleftrightarrow} Q(i) *\lceil\triangleright Q(i)\rceil
\end{aligned}
$$

Lemma 6.2. $\quad r \stackrel{1 / 2}{\Longleftrightarrow} P *\lfloor P\rfloor * \operatorname{resource}(\mathcal{I}, \mathcal{W} \uplus\{r\}) \sqsubseteq \operatorname{resource}(\mathcal{I}, \mathcal{W})$ Proof.

$$
\begin{aligned}
& r \stackrel{1 / 2}{\Longleftrightarrow} R *\lfloor R\rfloor * \operatorname{resource}(\mathcal{I}, \mathcal{W} \uplus\{r\}) \\
& \text { Definition of resource. } \\
& \sqsubseteq \quad r \stackrel{1 / 2}{\Longleftrightarrow} R *\lfloor R\rfloor * \\
& \left(\begin{array}{c}
\exists Q: \mathcal{I} \rightarrow \text { Prop, } R: \mathcal{W} \uplus\{r\} \rightarrow \text { Prop. } \\
\circledast_{i \in \mathcal{I}} \cdot i \stackrel{1 / 2}{\longmapsto} Q(i) * \circledast_{w \in \mathcal{W} \uplus\{r\}} \cdot w \stackrel{1 / 2}{\longmapsto} R(w) \\
*\left\lfloor\left(\triangleright \circledast_{w \in \mathcal{W} \uplus\{r\}} \cdot R(w)\right) \rightarrow \triangleright \circledast_{i \in \mathcal{I}} \cdot\lceil Q(i)\rceil\right\rfloor
\end{array}\right)
\end{aligned}
$$

Property (3), monotonicity of $\triangleright$, monotonicity of $\lfloor-\rfloor$.

$\sqsubseteq \quad \exists P$ : Prop. $r \stackrel{1 / 2}{\Longleftrightarrow} R *\lfloor\triangleright R\rfloor *(\lfloor\triangleright R\rfloor \Rightarrow\lfloor\triangleright P\rfloor)$

$$
\left(\begin{array}{c}
\exists Q: \mathcal{I} \rightarrow \text { Prop, } R: \mathcal{W} \rightarrow \text { Prop. } \\
r \stackrel{1 / 2}{\Longleftrightarrow} P * \circledast_{i \in \mathcal{I}} \cdot i \stackrel{1 / 2}{\Longleftrightarrow} Q(i) * \circledast_{w \in \mathcal{W}} \cdot w \stackrel{1 / 2}{\Longleftrightarrow} R(w) \\
*\left\lfloor\left(\triangleright P * \triangleright \circledast_{w \in \mathcal{W}} \cdot R(w)\right) * \triangleright \circledast_{i \in \mathcal{I}}\lceil Q(i)\rceil\right\rfloor
\end{array}\right)
$$

Modus ponens.

$$
\begin{aligned}
& \sqsubseteq \quad \exists P \text { : Prop. } r \stackrel{1 / 2}{\Longleftrightarrow} R *\lfloor\triangleright P\rfloor * \\
& \left(\begin{array}{c}
\exists Q: \mathcal{I} \rightarrow \text { Prop, } R: \mathcal{W} \rightarrow \text { Prop. } \\
r \stackrel{1 / 2}{\Longleftrightarrow} P * \circledast_{i \in \mathcal{I}} \cdot i \stackrel{1 / 2}{\Longrightarrow} Q(i) * \circledast_{w \in \mathcal{W}} \cdot w \stackrel{1 / 2}{\longmapsto} R(w) \\
*\left\lfloor\left(\triangleright P * \triangleright \circledast_{w \in \mathcal{W}} \cdot R(w)\right) \rightarrow \triangleright \circledast_{i \in \mathcal{I}} \cdot\lceil Q(i)\rceil\right\rfloor
\end{array}\right) \\
& \text { Combine }\lfloor-\rfloor \text {, modus ponens for } * \text {, weakening. } \\
& \sqsubseteq \quad \exists Q: \mathcal{I} \rightarrow \text { Prop, } R: \mathcal{W} \rightarrow \text { Prop. } \\
& \circledast_{i \in \mathcal{I}} \cdot i \stackrel{1 / 2}{\Longrightarrow} Q(i) * \circledast_{w \in \mathcal{W}} \cdot w \stackrel{1 / 2}{\Longleftrightarrow} R(w) \\
& *\left\lfloor\left(\triangleright \circledast_{w \in \mathcal{W}} \cdot R(w)\right) * \triangleright \circledast_{i \in \mathcal{I}} \cdot\lceil Q(i)\rceil\right\rfloor \\
& \sqsubseteq \operatorname{resource}(\mathcal{I}, \mathcal{W})
\end{aligned}
$$

Lemma 6.3. chainres $(r s) \wedge \operatorname{wf}(r s) \wedge \operatorname{ctrue}(r s)$

$$
\sqsubseteq \exists r s^{\prime} . \text { chainres }\left(r s^{\prime}\right) \wedge \operatorname{cconf}\left(r s^{\prime}\right) \wedge r s \stackrel{p r}{\rightarrow}^{*} r s^{\prime} \wedge \mathrm{wf}\left(r s^{\prime}\right)
$$


PROOF. We perform a sequence of smaller view-shifts corresponding to converting each cnode in turn, starting with the earliest element in the chain:

$$
P_{0} \sqsubseteq P_{1} \sqsubseteq P_{2} \sqsubseteq \ldots \sqsubseteq P_{n}
$$

Here $n$ is the length of $r s$ and the subscript $1,2,3 \ldots$ denotes the length of suffix of $r s$ which has been checked. We write $r s[a, b]$ for the subsequence of $r s$ from element $a$ to element $b$, inclusive of both. Thus the inductive invariant is:

$$
\begin{aligned}
P_{i} \triangleq \quad & \exists r s^{\prime} . \text { chainres }\left(r s[0, n-i] \cdot r s^{\prime}\right) \wedge \operatorname{cconf}\left(r s^{\prime}\right) \\
& \wedge r s[(n-i)+1, n] \stackrel{p r}{\rightarrow}^{*} r s^{\prime} \wedge \operatorname{wf}\left(r s[0,(n-i)] \cdot r s^{\prime}\right)
\end{aligned}
$$

The base case of the proof is simple. If $i=0$ take $r s^{\prime}$ to be empty and the invariant follows trivially from the premise. Let us assume that $i>0$. We reason as follows to pull out the intermediate chain node:

$$
\begin{aligned}
& \exists r s^{\prime} . \text { chainres }\left(r s[0, n-i] \cdot r s^{\prime}\right) \wedge \operatorname{cconf}\left(r s^{\prime}\right) \wedge r s[(n-i)+1, n] \stackrel{p r}{\rightarrow} * r s^{\prime} \wedge \operatorname{wf}\left(r s[0, n-i] \cdot r s^{\prime}\right) \\
& \quad \sqsubseteq \\
& \exists r s^{\prime}, s \cdot \operatorname{chainres}(r s[0, n-(i+1)]) * \operatorname{resource}(s \cdot \mathcal{I}, s . \mathcal{W}) * \text { chainres }\left(r s^{\prime}\right) \\
& \left.\wedge \operatorname{cconf}\left(r s^{\prime}\right) \wedge r s[n-i, n] \stackrel{p r}{\rightarrow} s \cdot r s^{\prime} \wedge \operatorname{wf}(r s[0, n-(i+1))] \cdot s \cdot r s^{\prime}\right)
\end{aligned}
$$

If $s . \mathcal{W}=\emptyset$ then we are done. Otherwise, we induct on the size of $\mathcal{W}$, showing that it can be reduced to $\emptyset$ by classical entailment. (Recall that by definition $\mathcal{W}$ is finite.)

Pick an element $w \in \mathcal{W}$. Since the chain is well-formed, the region identifier $w$ must also be a member of some set $s^{\prime}$. I for an earlier element $s^{\prime} \in r s^{\prime}$. If $w$ is a member of $s^{\prime}$.I for multiple $s^{\prime}$, we pick the first such $s^{\prime} \in r s^{\prime}$. Since cconf holds for $r s^{\prime}$ it follows that $s^{\prime} . \mathcal{W}=\emptyset$. Thus, there exists $r s_{1}^{\prime}, r s_{2}^{\prime}$ and $s^{\prime}$ such that $w \in s^{\prime} \cdot \mathcal{I}, s^{\prime} \cdot \mathcal{W}=\emptyset, r s^{\prime}=r s_{1}^{\prime} \cdot s^{\prime} \cdot r s_{2}^{\prime}$ and $\forall x \in r s_{1}^{\prime} . w \notin x . \mathcal{I}$. By the definition of resource, there exists a saved proposition

$w \stackrel{1 / 2}{\Longleftrightarrow} R$ inside resource $(s . \mathcal{I}, s . \mathcal{W})$. By the same definition, the saved proposition $w \stackrel{1 / 2}{\Longrightarrow} P$ and resource $\triangleright P$ must be included in resource $\left(s^{\prime} . \mathcal{I}, \emptyset\right)$. We move the resource from one node to the other, and delete the saved proposition using weakening:

$$
\begin{aligned}
& \operatorname{resource}(s . \mathcal{I}, s . \mathcal{W}) * \operatorname{chainres}\left(r s_{1}^{\prime}\right) * \operatorname{resource}\left(s^{\prime} \cdot \mathcal{I}, \emptyset\right) * \text { chainres }\left(r s_{2}^{\prime}\right) \\
& \quad \sqsubseteq \\
& \operatorname{resource}(s . \mathcal{I}, s . \mathcal{W} \backslash\{w\}) * \operatorname{chainres}\left(r s_{1}^{\prime}\right) * \operatorname{resource}\left(s^{\prime} . \mathcal{I} \backslash\{w\}, \emptyset\right) * \text { chainres }\left(r s_{2}^{\prime}\right)
\end{aligned}
$$

Let $r s^{\prime \prime}$ denote $r s_{1}^{\prime} \cdot s^{\prime}\left[\mathcal{I} \mapsto s^{\prime} \cdot \mathcal{I} \backslash\{w\}\right] \cdot r s_{2}^{\prime}$. By definition of $\stackrel{\mathcal{W}}{\rightarrow}$ it thus follows that

$$
r s[n-i, n] \stackrel{p r}{\rightarrow} s \cdot r s^{\prime} \stackrel{\mathcal{W}}{\rightarrow} s[\mathcal{W} \mapsto s . \mathcal{W} \backslash\{w\}] \cdot r s^{\prime \prime}
$$

Hence, by Lemma B.1 it follows that

$$
\left.\operatorname{wf}(r s[0, n-(i+1))] \cdot s[\mathcal{W} \mapsto s . \mathcal{W} \backslash\{w\}] \cdot r s^{\prime \prime}\right)
$$

The result is that the assertion is rewritten as follows:

$$
\begin{array}{r}
\sqsubseteq \quad \exists r s^{\prime}, s \cdot \operatorname{chainres}(r s[0, n-(i+1)]) * \operatorname{resource}(s \cdot \mathcal{I}, s . \mathcal{W} \backslash\{w\}) * \operatorname{chainres}\left(r s^{\prime}\right) \\
\left.\wedge \operatorname{cconf}\left(r s^{\prime}\right) \wedge r s[n-i, n] \stackrel{p r}{\rightarrow}^{*} s \cdot r s^{\prime} \wedge \operatorname{wf}(r s[0, n-(i+1))] \cdot s \cdot r s^{\prime}\right)
\end{array}
$$

Thus we have rewritten $s . \mathcal{W}$ into a smaller set. By inducting on the size of this set we can get to the point where $\mathcal{W}^{\prime}=\emptyset$. This allows us to complete one step of the outer induction, which completes the inductive proof.

LEMMA B.1.

$$
\operatorname{wf}(r s) \wedge r s \stackrel{\mathcal{W}}{\rightarrow} r s^{\prime} \Rightarrow\left(\text { available }(r s)=\text { available }\left(r s^{\prime}\right) \wedge \operatorname{wf}\left(r s^{\prime}\right)\right)
$$


Proof. By definition of $r s \stackrel{\mathcal{W}}{\rightarrow} r s^{\prime}$ there exists $r s_{1}, r s_{2}, r s_{3}, s_{1}, s_{2}$ and $w$ such that

$$
\begin{array}{r}
r s=r s_{1} \cdot s_{1} \cdot r s_{2} \cdot s_{2} \cdot r s_{3}, \quad w \in s_{2} \cdot \mathcal{I}, \quad w \in s_{1} \cdot \mathcal{W}, \\
r s^{\prime}=r s_{1} \cdot\left(s_{1} \triangleleft_{\mathcal{W}}(\bullet \backslash w)\right) \cdot r s_{2} \cdot\left(s_{2} \triangleleft_{\mathcal{I}}(\bullet \backslash w)\right) \cdot r s_{3}
\end{array}
$$

Since $s_{2} . \mathcal{W} \cap s_{2} \cdot \mathcal{I}=\emptyset$ and $\operatorname{wf}\left(s_{2} \cdot r s_{3}\right)$ it follows that $w \notin\left(s_{2} \cdot \mathcal{W} \cup\right.$ available $\left.\left(r s_{3}\right)\right)$ and $\forall s \in r s_{2} . w \notin s . \mathcal{I}$. Thus,

$$
\begin{aligned}
\operatorname{available}\left(\left(s_{2} \triangleleft_{\mathcal{I}}(\bullet \backslash w)\right) \cdot r s_{3}\right) & =\left(\text { available }\left(r s_{3}\right) \backslash s_{2} . \mathcal{W}\right) \uplus\left(s_{2} \cdot \mathcal{I} \backslash\{w\}\right) \\
& =\text { available }\left(s_{2} \cdot r s_{3}\right) \backslash\{w\}
\end{aligned}
$$

Since $w \in s_{1} . \mathcal{W}$ and $\operatorname{wf}\left(s_{1} \cdot r s_{2} \cdot s_{2} \cdot r s_{3}\right)$ it follows that $w \in$ available $\left(r s_{2} \cdot s_{2} \cdot r s_{3}\right)$. Hence, since $\forall s \in r s_{2} . w \notin s$.I it follows that $\forall s \in r s_{2} . w \notin s . \mathcal{W}$. Thus,

$$
\begin{aligned}
& \text { available }\left(\left(s_{1} \triangleleft_{\mathcal{W}}(\bullet \backslash w)\right) \cdot r s_{2} \cdot\left(s_{2} \mathbf{\cup}_{\mathcal{I}}(\bullet \backslash w)\right) \cdot r s_{3}\right) \\
= & \left(\text { available }\left(r s_{2} \cdot\left(s_{2} \bullet_{\mathcal{I}}(\bullet \backslash w)\right) \cdot r s_{3}\right) \backslash\left(s_{1} \cdot \mathcal{W} \backslash\{w\}\right)\right) \uplus s_{1} . \mathcal{I} \\
= & \left(\left(\text { available }\left(r s_{2} \cdot s_{2} \cdot r s_{3}\right) \backslash\{w\}\right) \backslash\left(s_{1} . \mathcal{W} \backslash\{w\}\right)\right) \uplus s_{1} \cdot \mathcal{I} \\
= & \left(\text { available }\left(r s_{2} \cdot s_{2} \cdot r s_{3}\right) \backslash s_{1} \cdot \mathcal{W}\right) \uplus s_{1} \cdot \mathcal{I} \\
= & \text { available }\left(s_{1} \cdot r s_{2} \cdot s_{2} \cdot r s_{3}\right)
\end{aligned}
$$

from which it follows easily that available $(r s)=$ available $\left(r s^{\prime}\right)$. To show that $\mathrm{wf}\left(r s^{\prime}\right)$ we must also show that

$$
\begin{aligned}
s_{1} \cdot \mathcal{W} \backslash\{w\} & \subseteq \text { available }\left(r s_{2} \cdot\left(s_{2} \triangleleft_{\mathcal{I}}(\bullet \backslash w)\right) \cdot r s_{3}\right) \\
& =\text { available }\left(r s_{2} \cdot s_{2} \cdot r s_{3}\right) \backslash\{w\}
\end{aligned}
$$

and $s_{2} . \mathcal{W} \subseteq$ available $\left(r s_{3}\right)$ both of which follow easily from the assumption that wf $(r s)$. It remains to show that all sets $\mathcal{I}$ for the chain are pairwise disjoint, and likewise for all sets $\mathcal{W}$. As we have only removed identifiers, this is satisfied trivially.

Lemma 6.4. resource $\left(\mathcal{I} \uplus r_{2}, \emptyset\right) * r_{2} \stackrel{1 / 2}{\Longleftrightarrow} P \sqsubseteq \operatorname{resource}(\mathcal{I}, \emptyset) *\lceil\triangleright P\rceil$

ProOF.

$$
\begin{aligned}
& \operatorname{resource}\left(\mathcal{I} \uplus r_{2}, \emptyset\right) * r_{2} \stackrel{1 / 2}{\Longleftrightarrow} P \\
& \text { Lemma 6.1. } \\
& \sqsubseteq \quad r_{2} \stackrel{1 / 2}{\Longleftrightarrow} P * \circledast_{i \in \mathcal{I} \uplus r_{2}} \cdot \exists R . i \stackrel{1 / 2}{\Longleftrightarrow} R *\lceil\triangleright R\rceil \\
& \text { Pull out resource for identifier } r_{2} \text {. } \\
& \sqsubseteq \quad r_{2} \stackrel{1 / 2}{\Longleftrightarrow} P * \exists R^{\prime} . r_{2} \stackrel{1 / 2}{\Longleftrightarrow} R^{\prime} *\left\lceil\triangleright R^{\prime}\right\rceil * \circledast \circledast_{i \in \mathcal{I}} \cdot \exists R . i \stackrel{1 / 2}{\Longleftrightarrow} R *\lceil\triangleright R\rceil \\
& \text { Property (3), monotonicity of }\lceil-\rceil \text {. } \\
& \sqsubseteq \quad r_{2} \stackrel{1 / 2}{\Longleftrightarrow} P * \exists R^{\prime} .\left(\left\lceil\triangleright R^{\prime}\right\rceil \Rightarrow\lceil\triangleright P\rceil\right) * r_{2} \stackrel{1 / 2}{\Longleftrightarrow} R^{\prime} *\left\lceil\triangleright R^{\prime}\right\rceil * \circledast_{i \in \mathcal{I}} \cdot \exists R . i \stackrel{1 / 2}{\Longleftrightarrow} R *\lceil\triangleright R\rceil \\
& \text { Modus ponens, weakening. } \\
& \sqsubseteq\lceil\triangleright P\rceil * \circledast \circledast_{i \in \mathcal{I}} \cdot \exists R . i \stackrel{1 / 2}{\Longrightarrow} R *\lceil\triangleright R\rceil \\
& \text { Definition of resource. } \\
& \sqsubseteq\lceil\triangleright P\rceil * \operatorname{resource}(\mathcal{I}, \emptyset)
\end{aligned}
$$

LEMMA 6.7.

$$
\left\{\operatorname{oreg}(r,\{\text { Chain }(r s) \mid x \in r s\}) * \operatorname{oreg}\left(r^{\prime},\left\{\text { Chain }\left(r s^{\prime}\right) \mid x \in r s^{\prime}\right\}\right)\right\} \quad\langle\text { skip }\rangle \quad\left\{r=r^{\prime}\right\}
$$


Proof. Prove this by case-splitting on whether the two regions are equal. Suppose the two are equal - then the specification is proved. If they are unequal, we prove this leads to a contradiction by opening both regions and examining their contents. Each region asserts exclusive ownership of heap cell $x . l o c$ which leads to a contradiction. Therefore the postcondition is false, allowing us to prove any post-condition.

Lemma 6.8. $\operatorname{resource}(\mathcal{I}, \mathcal{W} \uplus\{r\}) * r \stackrel{1 / 2}{\Longleftrightarrow} S * r^{\prime} \stackrel{1 / 2}{\Longleftrightarrow} T_{1}$

$$
\sqsubseteq \quad \exists r^{\prime \prime} . \operatorname{resource}\left(\mathcal{I}, \mathcal{W} \uplus\left\{r^{\prime}, r^{\prime \prime}\right\}\right) * r^{\prime \prime} \supseteqq\left(T_{1} * S\right)
$$

Proof. First we construct a new saved proposition $r^{\prime \prime}$ such that $r^{\prime \prime} \Leftrightarrow\left(T_{1} * S\right)$. Now it suffices to prove

$$
\begin{aligned}
& \operatorname{resource}(\mathcal{I}, \mathcal{W} \uplus\{r\}) * r \stackrel{1 / 2}{\Longleftrightarrow} S * r^{\prime} \stackrel{1 / 2}{\Longleftrightarrow} T_{1} * r^{\prime \prime} \stackrel{1 / 2}{\Longleftrightarrow} T_{2} \wedge \operatorname{valid}\left(T_{1} * T_{2} \Rightarrow S\right) \\
& \sqsubseteq \operatorname{resource}\left(\mathcal{I}, \mathcal{W} \uplus\left\{r^{\prime}, r^{\prime \prime}\right\}\right) \\
& r \stackrel{1 / 2}{\Longleftrightarrow} S * r^{\prime} \stackrel{1 / 2}{\Longleftrightarrow} T_{1} * r^{\prime \prime} \stackrel{1 / 2}{\Longleftrightarrow} T_{2} \wedge \operatorname{valid}\left(T_{1} * T_{2} \Rightarrow S\right) * \\
& \left(\begin{array}{c}
\exists Q: \mathcal{I} \rightarrow \text { Prop, } R: \mathcal{W} \uplus\{r\} \rightarrow \text { Prop. } \\
\circledast_{i \in \mathcal{I}} \cdot i \stackrel{1 / 2}{\Longrightarrow}^{\longmapsto}(i) * \circledast_{w \in \mathcal{W} \uplus\{r\}} \cdot w \stackrel{1 / 2}{\Longrightarrow} R(w) \\
*\left\lfloor\left(\triangleright \circledast_{w \in \mathcal{W} \uplus\{r\}} \cdot R(w)\right) * \triangleright \circledast_{i \in \mathcal{I}} \cdot\lceil Q(i)\rceil\right\rfloor
\end{array}\right) \\
& \text { Rearrange } \\
& \sqsubseteq \exists P: \text { Prop, } Q: \mathcal{I} \rightarrow \text { Prop, } R: \mathcal{W} \uplus\left\{r^{\prime}, r^{\prime \prime}\right\} \rightarrow \text { Prop. } \\
& \operatorname{valid}\left(R\left(r^{\prime}\right) * R\left(r^{\prime \prime}\right) \Rightarrow S\right) \wedge r \stackrel{1 / 2}{\Longleftrightarrow} S * r \stackrel{1 / 2}{\Longleftrightarrow} P \\
& * \circledast_{i \in \mathcal{I}} \cdot i \stackrel{1 / 2}{\Longrightarrow} Q(i) * \circledast_{w \in \mathcal{W} \uplus\left\{r^{\prime}, r^{\prime \prime}\right\}} \cdot w \stackrel{1 / 2}{\Longrightarrow} R(w) \\
& \left.*\left\lfloor\triangleright P * \triangleright \circledast_{w \in \mathcal{W}} \cdot R(w)\right) * \triangleright \circledast_{i \in \mathcal{I}} \cdot\lceil Q(i)\rceil\right\rfloor \\
& \text { Property (3), SMono and distributing } \triangleright \text { over } \Longrightarrow \text {. }
\end{aligned}
$$

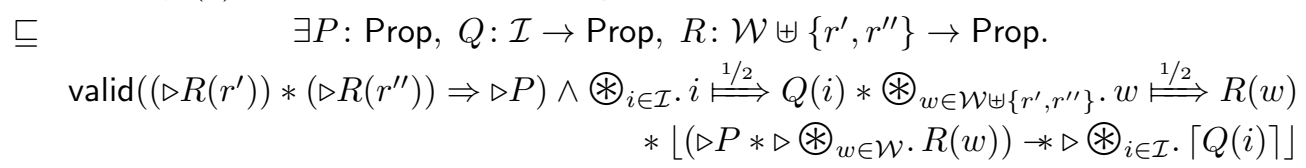

$$
\begin{aligned}
& \sqsubseteq \quad \exists Q: \mathcal{I} \rightarrow \text { Prop, } R: \mathcal{W} \uplus\left\{r^{\prime}, r^{\prime \prime}\right\} \rightarrow \text { Prop. } \\
& \circledast_{i \in \mathcal{I}} \cdot i \stackrel{1 / 2}{\Longleftrightarrow} Q(i) * \circledast_{w \in \mathcal{W} \uplus\left\{r^{\prime}, r^{\prime \prime}\right\}} \cdot w \stackrel{1 / 2}{\Longleftrightarrow} R(w) \\
& *\left\lfloor\left(\left(\triangleright R\left(r^{\prime}\right)\right) *\left(\triangleright R\left(r^{\prime \prime}\right)\right) * \triangleright \circledast_{w \in \mathcal{W}} \cdot R(w)\right) * \triangleright \circledast_{i \in \mathcal{I}} \cdot\lceil Q(i)\rceil\right\rfloor \\
& \circledast_{i \in \mathcal{I}} \cdot i \stackrel{1 / 2}{\Longrightarrow} Q(i) * \circledast_{w \in \mathcal{W} \uplus\left\{r^{\prime}, r^{\prime \prime}\right\}} \cdot w \stackrel{1 / 2}{\Longrightarrow} R(w) \\
& \left.*\left\lfloor\triangleright \circledast_{w \in \mathcal{W} \uplus\left\{r^{\prime}, r^{\prime \prime}\right\}} . R(w)\right) * \triangleright \circledast_{i \in \mathcal{I}} \cdot\lceil Q(i)\rceil\right\rfloor
\end{aligned}
$$

LEMMA B.2.

$$
\begin{aligned}
r_{2} \stackrel{1 / 2}{\Longleftrightarrow} P *\left\lfloor\lceil P\rceil *\left(P_{1} * P_{2}\right)\right\rfloor * r_{3} \stackrel{1 / 2}{\Longleftrightarrow} P_{1} * r_{4} \stackrel{1 / 2}{\Longleftrightarrow} P_{2} * \operatorname{resource}\left(\mathcal{I} \uplus\left\{r_{2}\right\}, \mathcal{W}\right) \\
\\
\sqsubseteq \operatorname{resource}\left(\mathcal{I} \uplus\left\{r_{3}, r_{4}\right\}, \mathcal{W}\right)
\end{aligned}
$$


Proof.

$r_{2} \stackrel{1 / 2}{\Longleftrightarrow} P *\left\lfloor\lceil P\rceil *\left(P_{1} * P_{2}\right)\right\rfloor * r_{3} \stackrel{1 / 2}{\Longleftrightarrow} P_{1} * r_{4} \stackrel{1 / 2}{\Longleftrightarrow} P_{2} * \operatorname{resource}\left(\mathcal{I} \uplus\left\{r_{2}\right\}, \mathcal{W}\right)$

Definition of resource.

$$
\begin{aligned}
& \sqsubseteq r_{2} \stackrel{1 / 2}{\Longleftrightarrow} P *\left\lfloor\lceil P\rceil *\left(P_{1} * P_{2}\right)\right\rfloor * r_{3} \stackrel{1 / 2}{\Longleftrightarrow} P_{1} * r_{4} \stackrel{1 / 2}{\Longrightarrow} P_{2} \\
& \left(\begin{array}{c}
\exists Q: \mathcal{I} \uplus\left\{r_{2}\right\} \rightarrow \text { Prop, } R: \mathcal{W} \rightarrow \text { Prop. } \\
\circledast_{i \in \mathcal{I} \uplus\left\{r_{2}\right\}} \cdot i \stackrel{1 / 2}{\longmapsto} Q(i) * \circledast_{w \in \mathcal{W}} \cdot w \stackrel{1 / 2}{\Longleftrightarrow} R(w) \\
*\left(\left\lfloor\left(\triangleright \circledast_{w \in \mathcal{W}} \cdot R(w)\right) \rightarrow \triangleright \circledast_{i \in \mathcal{I} \uplus\left\{r_{2}\right\}} \cdot\lceil Q(i)\rceil\right\rfloor\right)
\end{array}\right)
\end{aligned}
$$

Pull out $r_{2}, \triangleright$ mono w.r.t. $*$, property (4).

$$
\begin{aligned}
& \sqsubseteq \quad r_{2} \stackrel{1 / 2}{\Longleftrightarrow} P * r_{3} \stackrel{1 / 2}{\Longleftrightarrow} P_{1} * r_{4} \stackrel{1 / 2}{\Longrightarrow} P_{2} \\
& \left(\begin{array}{c}
\exists Q: \mathcal{I} \uplus\left\{r_{2}\right\} \rightarrow \text { Prop, } R: \mathcal{W} \rightarrow \text { Prop. } \\
r_{2} \stackrel{1 / 2}{\longmapsto} Q\left(r_{2}\right) * \circledast_{i \in \mathcal{I}} \cdot i \stackrel{1 / 2}{\longmapsto} Q(i) * \circledast_{w \in \mathcal{W}} \cdot w \stackrel{1 / 2}{\longmapsto} R(w) \\
\left.*\left\lfloor\left(\triangleright \circledast_{w \in \mathcal{W}} \cdot R(w)\right) * \triangleright\left(P_{1} * P_{2} * \circledast_{i \in \mathcal{I}} \cdot\lceil Q(i)\rceil\right)\right\rfloor\right)
\end{array}\right)
\end{aligned}
$$

Fold $r_{3}, r_{4}$ into $\mathcal{I}$, weaken with $\left\lceil_{-}\right\rceil$, weakening.

$$
\begin{gathered}
\exists Q: \mathcal{I} \uplus r_{2} \rightarrow \text { Prop, } R: \mathcal{W} \rightarrow \text { Prop. } \\
\circledast_{i \in \mathcal{I} \uplus\left\{r_{3}, r_{4}\right\}} \cdot i \stackrel{1 / 2}{\longmapsto} Q(i) * \circledast_{w \in \mathcal{W}} \cdot w \stackrel{1 / 2}{\longmapsto} R(w) \\
\quad\left\lfloor\left\lfloor\left(\triangleright \circledast_{w \in \mathcal{W}} \cdot R(w)\right) * \circledast_{i \in \mathcal{I} \uplus\left\{r_{3}, r_{4}\right\}} \cdot\lceil Q(i)\rceil\right\rfloor\right) \\
\text { Definition of resource. }
\end{gathered}
$$

$\sqsubseteq \operatorname{resource}\left(\mathcal{I} \uplus\left\{r_{3}, r_{4}\right\}, \mathcal{W}\right)$

Lemma 6.9. $r_{2} \in r s(x) . \mathcal{I} \wedge r_{2} \stackrel{1 / 2}{\Longleftrightarrow} P *\left\lfloor\lceil P\rceil *\left(P_{1} * P_{2}\right)\right\rfloor *$ chainres $(r s)$

$$
\begin{array}{r}
\sqsubseteq \quad \exists r s^{\prime}, r_{3}, r_{4} \cdot r_{3}, r_{4} \notin r s \wedge r s^{\prime}=r s \triangleleft_{x} \triangleleft_{\mathcal{I}}\left(\bullet \backslash r_{2}\right) \uplus\left\{r_{3}, r_{4}\right\} \wedge \\
r_{3} \stackrel{1 / 2}{\Longleftrightarrow} P_{1} * r_{4} \stackrel{1 / 2}{\Longleftrightarrow} P_{2} * \operatorname{chainres}\left(r s^{\prime}\right)
\end{array}
$$


Proof.

$r_{2} \in r s(x) \cdot \mathcal{I} \wedge r_{2} \stackrel{1 / 2}{\Longleftrightarrow} P *\left\lfloor\lceil P\rceil *\left(P_{1} * P_{2}\right)\right\rfloor *$ chainres $(r s)$

Make saved propositions, fresh by construction.

$\sqsubseteq r_{2} \in r s(x) \cdot \mathcal{I} \wedge r_{2} \stackrel{1 / 2}{\Longrightarrow} P *\left\lfloor\lceil P\rceil *\left(P_{1} * P_{2}\right)\right\rfloor *$ chainres $(r s) *$

$\exists r_{3}, r_{4} \cdot r_{3} \stackrel{1}{\Rightarrow} P_{1} * r_{4} \stackrel{1}{\Rightarrow} P_{2} \wedge r_{3}, r_{4} \notin r s$

Pull out resource predicate for $x$.

$\sqsubseteq \exists r s_{1}, r s_{2} \cdot r_{2} \in r s(x) \cdot \mathcal{I} \wedge r_{2} \stackrel{1 / 2}{\Longleftrightarrow} P *\left\lfloor\lceil P\rceil *\left(P_{1} * P_{2}\right)\right\rfloor *$

chainres $\left(r s_{1}\right) *$ resource $(r s(x) . \mathcal{I}, r s(x) . \mathcal{W} \uplus\{r s(x)$.res $\mid r s(x)$.flg $=0\}) *$ chainres $\left(r s_{2}\right) *$

$\exists r_{3}, r_{4} \cdot r_{3} \stackrel{1}{\Rightarrow} P_{1} * r_{4} \stackrel{1}{\Rightarrow} P_{2} \wedge r_{3}, r_{4} \notin r s \wedge r s=r s_{1} \cdot r s(x) \cdot r s_{2}$

Apply Lemma B.2.

$\sqsubseteq \exists r s_{1}, r s_{2}, r_{3}, r_{4} \cdot r_{2} \in r s(x) \cdot \mathcal{I} \wedge r_{2} \stackrel{1 / 2}{\Longleftrightarrow} P *\left\lfloor\lceil P\rceil *\left(P_{1} * P_{2}\right)\right\rfloor *$

chainres $\left(r s_{1}\right) *$ resource $\left(\left(r s(x) . \mathcal{I} \backslash r_{2}\right) \uplus\left\{r_{3}, r_{4}\right\}, r s(x) . \mathcal{W} \uplus\{r s(x)\right.$.res $\mid r s(x)$.flg $\left.=0\}\right) *$

chainres $\left(r s_{2}\right) * r_{3} \stackrel{1 / 2}{\Longleftrightarrow} P_{1} * r_{4} \stackrel{1 / 2}{\Longleftrightarrow} P_{2} \wedge r_{3}, r_{4} \notin r s \wedge r s=r s_{1} \cdot r s(x) \cdot r s_{2}$

Definition of chainres.

$\sqsubseteq \exists r s^{\prime}, r_{3}, r_{4} \cdot r_{3}, r_{4} \notin r s \wedge r s^{\prime}=r s \triangleleft_{x} \triangleleft_{\mathcal{I}}\left(\bullet \backslash r_{2}\right) \uplus\left\{r_{3}, r_{4}\right\} \wedge$

$r_{3} \stackrel{1 / 2}{\Longleftrightarrow} P_{1} * r_{4} \stackrel{1 / 2}{\Longleftrightarrow} P_{2} *$ chainres $\left(r s^{\prime}\right)$

\section{ICAP PROOF SYSTEM}

In this Appendix we introduce the formal iCAP proof system. The introduction is selfcontained, but does not cover the full iCAP proof system. In particular, certain iCAP features, such as guarded recursive predicates and phantom state are not necessary for the present paper, and have been dropped from the proof system.

\section{C.1. Syntax}

The proof system consists of two logics, an assertion logic and a specification logic, over a common simply-typed term language generated by the following grammar:

$\mathrm{M}, \mathrm{N}, \mathrm{P}, \mathrm{Q}, \mathrm{S}, \mathrm{T}, \mathrm{F}, \mathrm{R}::=$

$$
\begin{aligned}
& |\lambda \mathrm{x}: \tau . \mathrm{M}| \mathrm{M} N \mid \mathrm{x} \\
& |\perp| \mathrm{T}|\mathrm{M} \vee \mathrm{N}| \mathrm{M} \wedge \mathrm{M}|\mathrm{M} \Rightarrow \mathrm{N}| \forall \mathrm{x}: \tau . \mathrm{P}|\exists \mathrm{x}: \tau . \mathrm{P}| \mathrm{M}={ }_{\tau} \mathrm{N} \\
& |\mathrm{P} * \mathrm{Q}| \mathrm{P} * \mathrm{Q} \mid \text { emp }|\mathrm{M} . \mathrm{F} \mapsto \mathrm{N}| \mathrm{M}: \mathrm{N}|\operatorname{region}(\mathrm{R}, \mathrm{M}, \mathrm{N})|[\mathrm{M}]_{\mathrm{N}}^{\mathrm{R}} \mid \text { stable }(\mathrm{P}) \\
& \left|\mathrm{P} \sqsubseteq^{\mathrm{R}} \mathrm{Q}\right|(\Delta) .\{\mathrm{P}\} \overline{\mathrm{s}}\{\mathrm{Q}\}\left|(\Delta) .\langle\mathrm{P}\rangle \overline{\mathrm{s}}\langle\mathrm{Q}\rangle^{\mathrm{R}}\right| \mathrm{M} . \mathrm{N}:(\Delta) .\{\mathrm{P}\}\{\mathrm{x} . \mathrm{Q}\} \mid \mathrm{M}:(\Delta) .\{\mathrm{P}\}\{\mathrm{x} . \mathrm{Q}\} \\
& |\triangleright \mathrm{M}| \operatorname{valid}(\mathrm{P})|\operatorname{spec}(\mathrm{S})| \Delta_{X}(x)
\end{aligned}
$$

Here $X$ is an arbitrary set and $x$ an arbitrary element of $X$. The $\Delta_{X}(x)$ gives a shallow embedding of the meta-theory into iCAP. Correspondingly, the grammar of types (given below) features a type constructor $\Delta(X)$ for injecting arbirary sets into iCAP.

$$
\text { Types } \tau, \sigma::=1|\tau \rightarrow \sigma| \tau \times \sigma|\tau+\sigma| \mathcal{P}(\tau)|\Delta(X)| \text { Prop } \mid \text { Spec }
$$

In addition to the usual type-constructors, iCAP includes two proposition types - one for each logic - the Prop type for the assertion logic and the Spec type for the specification logic. Base-types for values, Val, state identifiers, Sld, action identifiers, Ald, region identifiers, RId, class names, Class, and field names, Field are just syntactic suger for injections of the 
corresponding set (e.g., Val is syntactic suger for $\Delta(\mathrm{Val})$ ). We will usually leave out the explicit injection for elements when reasoning about elements of these injected types.

Well-formed terms

$\Gamma ; \Delta \vdash \mathrm{M}: \tau$

The typing rules of the logic are given below. The rules have been split into standard higherorder logic typing rules, followed by iCAP specific typing rules. Terms are typed in a logical variable context, $\Gamma$, and program variable context, $\Delta$. Logical variables are used purely for specification purposes and may not appear free in the code of Hoare triples. Program variables may appear free in both the pre- and postcondition of Hoare triples and in the code snippet. The logical variable context, $\Gamma$, maps variables to types, while all variables in the program variable context, $\Delta$, have the type Val.

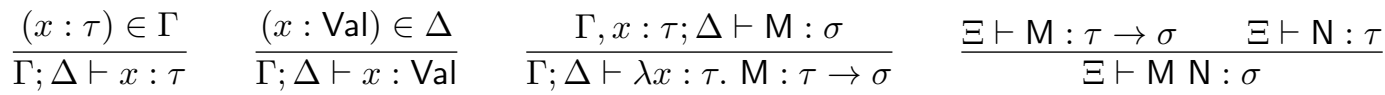

$$
\begin{aligned}
& \frac{p \in\{\perp, \top, \mathrm{emp}\}}{\Xi \vdash p: \text { Prop }} \quad \frac{\Xi \vdash \mathrm{P}: \text { Prop } \quad \Xi \vdash \mathrm{Q}: \text { Prop } \quad \text { op } \in\{\vee, \wedge, \Rightarrow, *, *\}}{\Xi \vdash \mathrm{P} \text { op } \mathrm{Q}: \text { Prop }} \\
& \frac{\Xi \vdash \mathrm{M}: \tau \quad \Xi \vdash \mathrm{N}: \tau}{\Xi \vdash \mathrm{M}={ }_{\tau} \mathrm{N}: \text { Prop }} \quad \frac{\Gamma, x: \tau ; \Delta \vdash \mathrm{P}: \text { Prop } \quad Q \in\{\exists, \forall\}}{\Gamma ; \Delta \vdash Q x: \tau . \mathrm{P}: \text { Prop }} \quad \frac{p \in\{\perp, \top\}}{\Gamma ;-\vdash p: \text { Spec }} \\
& \frac{\Gamma ;-\vdash \mathrm{S}: \mathrm{Spec} \quad \Gamma ;-\vdash \mathrm{T}: \mathrm{Spec} \quad o p \in\{\vee, \wedge, \Rightarrow\}}{\Gamma ;-\vdash \mathrm{S} \text { op } \mathrm{T}: \mathrm{Spec}} \quad \frac{\Gamma ;-\vdash \mathrm{M}: \tau \quad \Gamma ;-\vdash \mathrm{N}: \tau}{\Gamma ;-\vdash \mathrm{M}=_{\tau} \mathrm{N}: \mathrm{Spec}} \\
& \frac{\Gamma, x: \tau ;-\vdash \mathrm{S}: \operatorname{Spec} \quad Q \in\{\forall, \exists\}}{\Gamma ;-\vdash Q x: \tau \cdot \mathrm{S}: \mathrm{Spec}} \\
& \Gamma ;-\vdash Q x: \tau \text {. S : Spec } \\
& \frac{\Xi \vdash \mathrm{M}: \mathcal{P}(\mathrm{Sld}) \quad \Xi \vdash \mathrm{I}: \mathrm{Sld} \rightarrow \text { Prop } \quad \Xi \vdash \mathrm{T}: \text { Ald } \rightarrow \mathcal{P}(\mathrm{Sld} \times \mathrm{Sld}) \quad \Xi \vdash \mathrm{R}: \text { Rld }}{\Xi \vdash \operatorname{region}(\mathrm{M}, \mathrm{I}, \mathrm{T}, \mathrm{R}): \text { Prop }} \\
& \frac{\Xi \vdash \mathrm{A}: \text { Ald } \quad \Xi \vdash \mathrm{R}: \mathrm{Rld} \quad \Xi \vdash \mathrm{P}: \text { Perm }}{\Xi \vdash[\mathrm{A}]_{\mathrm{P}}^{\mathrm{R}}: \text { Prop }} \quad \frac{x \in X \quad X \in \text { obj(Sets })}{\Xi \vdash \Delta_{X}(x): \Delta(X)} \\
& \frac{\Xi \vdash \mathrm{M}: \text { Val } \quad \Xi \vdash \mathrm{C}: \text { Class }}{\Xi \vdash \mathrm{M}: \mathrm{C}: \text { Prop }} \frac{\Xi \vdash \mathrm{M}: \text { Val } \quad \Xi \vdash \mathrm{F}: \text { Field } \quad \Xi \vdash \mathrm{N}: \text { Val }}{\Xi \vdash \mathrm{M} . \mathrm{F} \mapsto \mathrm{N}: \text { Prop }} \\
& \frac{\Gamma ;-\vdash \mathrm{S}: \text { Spec }}{\Gamma ; \Delta \vdash \operatorname{spec}(\mathrm{S}): \text { Prop }} \quad \frac{\Gamma ;-\vdash \mathrm{P}: \text { Prop }}{\Gamma ;-\vdash \operatorname{valid}(\mathrm{P}): \text { Spec }} \quad \frac{\Xi \vdash \mathrm{P}: \text { Prop }}{\Xi \vdash \triangleright \mathrm{P}: \text { Prop }} \quad \frac{\Gamma ;-\vdash \mathrm{P}: \text { Prop }}{\Gamma ;-\vdash \operatorname{stable}(\mathrm{P}): \text { Spec }} \\
& \frac{\Gamma ;-\vdash \mathrm{R}: \mathcal{P}(\mathrm{Rld}) \quad \Gamma ;-\vdash \mathrm{P}: \text { Prop } \quad \Gamma ;-\vdash \mathrm{Q}: \text { Prop }}{\Gamma ;-\vdash \mathrm{P} \check{\mathrm{R}}^{\mathrm{R}} \mathrm{Q}: \text { Spec }} \quad \frac{\Gamma ;-\vdash \mathrm{S}: \text { Spec }}{\Gamma ;-\vdash \triangleright \mathrm{S}: \mathrm{Spec}} \\
& \frac{\Gamma ; \Delta \vdash \mathrm{P}: \operatorname{Prop} \quad \Gamma ; \Delta \vdash \mathrm{Q}: \operatorname{Prop} \quad \mathrm{FV}(\mathrm{s}) \subseteq \operatorname{vars}(\Delta)}{\Gamma ;-\vdash(\Delta) .\{\mathrm{P}\} \mathrm{s}\{\mathrm{Q}\}: \operatorname{Spec}} \\
& \frac{\Gamma ; \Delta \vdash \mathrm{P}: \text { Prop } \quad \Gamma ; \Delta \vdash \mathrm{Q}: \operatorname{Prop} \quad \Gamma ;-\vdash \mathrm{R}: \mathcal{P}(\mathrm{Rld}) \quad \mathrm{FV}(\mathrm{s}) \subseteq \operatorname{vars}(\Delta)}{\Gamma ;-\vdash(\Delta) .\langle\mathrm{P}\rangle \mathrm{s}\langle\mathrm{Q}\rangle^{\mathrm{R}}: \operatorname{Spec}}
\end{aligned}
$$




\section{C.2. Logics}

The iCAP proof system consists of two logics: an assertion logic for reasoning about the current state and a specification logic for reasoning about the behavior of programs. The specification logic is given by the specification entailment judgment $\Gamma \mid \Phi \vdash S$ where $S$ is a specification and $\Phi$ is a specification context. The assertion logic is given by the assertion entailment judgment $\Gamma ; \Delta|\Phi| \mathrm{P} \vdash \mathrm{Q}$ where $\mathrm{P}$ and $\mathrm{Q}$ are assertions and $\Phi$ is a specification context. The assertion entailment includes the specification context $\Phi$, to allow the use of assertion assumptions embedded in specifications.

C.2.1. Specification and assertion embeddings. The valid embedding is used to export axioms about abstract resources in library specifications to clients. For instance, the axioms about the channel order (duplication and transitivity) are implicitly expressed as validities about the channel order resource. The introduction and elimination rule for valid specifications is given below.

$$
\frac{\Gamma ;-|\Phi| \top \vdash \mathrm{P}}{\Gamma \mid \Phi \vdash \operatorname{valid}(\mathrm{P})} \quad \frac{\Gamma \mid \Phi \vdash \operatorname{valid}(\mathrm{P})}{\Gamma ;-|\Phi| \top \vdash \mathrm{P}}
$$

C.2.2. Later operator. The SLOB rule internalizes induction on step-indicies in the logic and is implicitly used when verifying mutually recursive methods. In particular, to verify a method call, it suffices to know that the body of the called method satisfies a given specification one step later, to know that the call satisfies the given specification now. Intuitively, calling a method uses one step in the operational semantics, before the method body starts executing.

$$
\begin{aligned}
& \text { SLOB SMONO LPOINTS } \\
& \frac{\Gamma \mid \Phi, \triangleright S \vdash S}{\Gamma \mid \Phi \vdash S} \quad \frac{\Gamma \mid \Phi \vdash\langle x . f \mapsto v\rangle c\langle Q\rangle^{\mathcal{E}}}{\Gamma \mid \Phi \vdash S \Rightarrow \triangleright S} \quad \frac{T \mid \Phi \vdash\langle\triangleright(x . f \mapsto v)\rangle c\langle Q\rangle^{\mathcal{E}}}{\Gamma \mid f}
\end{aligned}
$$

LBIN

$$
\frac{o p \in\{\wedge, \vee, *\}}{\Gamma ; \Delta|\Phi| \triangleright(\mathrm{P} \text { op } \mathrm{Q}) \dashv(\triangleright \mathrm{P}) \text { op }(\triangleright \mathrm{Q})}
$$

LWAND

$$
\overline{\Gamma ; \Delta|\Phi| \triangleright(\mathrm{P} * \mathrm{Q}) \vdash(\triangleright \mathrm{P}) *(\triangleright \mathrm{Q})}
$$

LIMPL

$$
\overline{\Gamma ; \Delta|\Phi| \triangleright(\mathrm{P} \Rightarrow \mathrm{Q}) \vdash(\triangleright \mathrm{P}) \Rightarrow(\triangleright \mathrm{Q})}
$$

LCEIL

$$
\overline{\Gamma ; \Delta|\Phi| \triangleright\lceil\mathrm{P}\rceil \dashv\lceil\triangleright \mathrm{P}\rceil}
$$

LFLOOR

$\overline{\Gamma ; \Delta|\Phi| \triangleright\lfloor\mathrm{P}\rfloor \dashv\lfloor\triangleright \mathrm{P}\rfloor}$

$$
\overline{\Gamma ; \Delta|\Phi| \operatorname{region}\left(X, T, I_{1}, r\right) * \operatorname{region}\left(Y, T, I_{2}, r\right) \vdash I_{1}(s) \Rightarrow I_{2}(s)}
$$

C.2.3. View-shifts. The view-shift relation includes standard assertion implication. In addition it is transitive and supports framing of stable frames. There is no implicit assumption 
that the pre- and postcondition of view-shifts is stable.

$$
\begin{aligned}
& \text { VTRANs } \\
& \frac{\Gamma\left|\Phi \vdash \mathrm{P} \sqsubseteq^{\mathcal{E}} \mathrm{Q} \quad \Gamma\right| \Phi \vdash \mathrm{Q} \sqsubseteq^{\mathcal{E}} \mathrm{R}}{\Gamma \mid \Phi \vdash \mathrm{Q} \sqsubseteq^{\mathcal{E}} \mathrm{R}}
\end{aligned}
$$

$$
\begin{aligned}
& \text { VALloc } \\
& \Gamma|\Phi \vdash \Gamma| \Phi \vdash \forall \alpha \in \text { Ald. } \forall x \in \text { SId } \times \text { SId. }(\triangleright T(\alpha)(x)) \Rightarrow T(\alpha)(x) \vee \triangleright \perp \\
& \Gamma \mid \Phi \vdash \mathcal{E} \text { is infinite } \Gamma \mid \Phi \vdash \forall n \in \mathcal{E} . P * \circledast \alpha \in A[\alpha]_{1}^{n} \Rightarrow \triangleright I(n)(x) \\
& \Gamma \mid \Phi \vdash \forall n \in \mathcal{E} . \forall s . \text { stable }(I(n)(s)) \quad \Gamma \mid \Phi \vdash A \cap B=\emptyset \\
& \hline \Gamma \mid \Phi \vdash \mathrm{P} \sqsubseteq^{\mathcal{E}} \exists n \in \mathcal{E} \text {. region }(\{x\}, T, I(n), n) * \circledast_{\alpha \in B}[\alpha]_{1}^{n}
\end{aligned}
$$

VOPEN

$$
\Gamma \mid \Phi \vdash \text { stable(P) } \quad \Gamma \mid \Phi \vdash \operatorname{stable}(\mathrm{Q})
$$

$\Gamma \mid \Phi \vdash \forall x \in X . f(x) \in Y$

$\Gamma \mid \Phi \vdash \forall x \in X .(x, f(x)) \in T(\alpha) \vee f(x)=x$

$\Gamma \mid \Phi \vdash \forall x \in X . \mathrm{P} * \triangleright I(x) *[\alpha]_{\pi}^{n} \sqsubseteq^{\mathcal{E}} \mathrm{Q} * \triangleright I(f(x))$

$$
\overline{\Gamma \mid \Phi \vdash \operatorname{region}(X, T, I, n) * \mathrm{P} *[\alpha]_{\pi}^{n} \sqsubseteq^{\mathcal{E} \uplus\{n\}} \operatorname{region}(Y, T, I, n) * \mathrm{Q}}
$$

The VALLOC rule presented above generalizes the region allocation rule presented in 4, by allowing the newly allocated region to immediately take ownership of action permissions on the newly allocated region $\left(\circledast_{\alpha \in A}[\alpha]_{1}^{n}\right)$. In addition, it allows the allocator to pick an infinite set $\mathcal{E}$ of region identifiers, from which the region identifier of the newly allocated region will be chosen. This is used to reason about inequality of region idenfiers, which is necessary when reasoning about nested region openings.

C.2.4. Atomic commands. All pre- and postconditions that appear in non-atomic Hoare triples are implicitly required to be stable.

$$
\begin{gathered}
\Gamma, \Delta \vdash \mathrm{P}, \mathrm{Q}: \text { Prop } \quad \text { atomic }(\mathrm{s}) \\
\Gamma, \Delta \mid \Phi \vdash \text { stable }(\mathrm{P}) \quad \Gamma, \Delta \mid \Phi \vdash \text { stable }(\mathrm{Q}) \\
\Gamma \mid \Phi \vdash(\Delta) .\langle\mathrm{P}\rangle \mathrm{s}\langle\mathrm{Q}\rangle^{\mathcal{E}} \\
\hline \Gamma \mid \Phi \vdash(\Delta) .\{\mathrm{P}\} \mathrm{s}\{\mathrm{Q}\} \\
\Gamma, \Delta \vdash \mathrm{P}, \mathrm{Q}: \operatorname{Prop} \quad \Gamma, \Delta \vdash \mathcal{E}_{1}, \mathcal{E}_{2}: \mathcal{P}(\mathrm{RId}) \\
\Gamma \mid \Phi \vdash(\Delta) .\langle\mathrm{P}\rangle \mathrm{s}\langle\mathrm{Q}\rangle^{\mathcal{E}_{1} \backslash \mathcal{E}_{2}} \\
\hline \Gamma \mid \Phi \vdash(\Delta) .\langle\mathrm{P}\rangle \mathrm{s}\langle\mathrm{Q}\rangle^{\mathcal{E}_{1}}
\end{gathered}
$$

Атоміс

$$
\Gamma, \Delta|\Phi \vdash \operatorname{stable}(\mathrm{P}) \quad \Gamma, \Delta| \Phi \vdash \forall y \text {. stable }(\mathrm{Q}(y))
$$

$\Gamma, \Delta \mid \Phi \vdash \forall x \in X .(x, f(x)) \in \overline{T(A)} \vee f(x)=x$

$\frac{\Gamma \mid \Phi \vdash \forall x \in X .(\Delta) \cdot\left\langle\mathrm{P} * \circledast_{\alpha \in A}[\alpha]_{g(\alpha)}^{n} * \triangleright I(x)\right\rangle c\langle\mathrm{Q}(x) * \triangleright I(f(x))\rangle^{\mathcal{E}}}{\Gamma \mid \Phi \vdash(\Delta) .\left\langle\mathrm{P} * \circledast_{\alpha \in A}[\alpha]_{g(\alpha)}^{n} * \operatorname{region}(X, T, I, n)\right\rangle}$

$c$

$$
\langle\exists x . \mathrm{Q}(x) * \operatorname{region}(\{f(x)\}, T, I, n)\rangle^{\mathcal{E} \uplus\{n\}}
$$




\section{C.2.5. Stability.}

$\frac{\Gamma \mid \Phi \vdash \forall \alpha \notin A . \forall x \in X . T(\alpha)(x) \subseteq X}{\Gamma \mid \Phi \vdash \text { stable }\left(\text { region }(X, T, I, n) * \circledast \alpha \in A[\alpha]_{1}^{n}\right)} \quad \overline{\Gamma \mid \Phi \vdash \operatorname{stable}(\top)} \quad \overline{\Gamma \mid \Phi \vdash \text { stable }(\perp)}$

$$
\overline{\Gamma \mid \Phi \vdash \text { stable(emp) }} \quad \overline{\Gamma \mid \Phi \vdash \text { stable(M.F } \mapsto \mathrm{N})} \quad \overline{\Gamma \mid \Phi \vdash \text { stable(M : N) }}
$$

$\frac{\Gamma \mid \Phi \vdash \text { stable }(\mathrm{P}) \quad \Gamma \mid \Phi \vdash \operatorname{stable}(\mathrm{Q})}{\Gamma \mid \Phi \vdash \operatorname{stable}(\mathrm{P} o p \mathrm{Q})} \quad \frac{o p \in\{\vee, \wedge, *\}}{\Gamma \mid \Phi \vdash \operatorname{stable}\left(\mathrm{M}={ }_{\tau} \mathrm{N}\right)}$

$\frac{\Gamma \mid \Phi \vdash \forall x: \tau \text {. stable }(\mathrm{P}(x)) \quad Q \in\{\forall, \exists\}}{\Gamma \mid \Phi \vdash \operatorname{stable}(Q x: \tau . \mathrm{P}(x))} \quad \frac{\Gamma \vdash \mathrm{S}: \text { Spec }}{\Gamma \mid \Phi \vdash \operatorname{stable}(\operatorname{spec}(\mathrm{S}))} \quad \frac{\Gamma \mid \Phi \vdash \operatorname{stable}(\mathrm{P})}{\Gamma \mid \Phi \vdash \operatorname{stable}(\triangleright \mathrm{P})}$

\section{C.2.6. Structural rules.}

VFrAME

$\Gamma\left|\Phi \vdash \mathrm{P} \sqsubseteq^{\mathcal{E}} \mathrm{Q} \quad \Gamma\right| \Phi \mid$ stable $(\mathrm{R})$

$$
\Gamma \mid \Phi \vdash \mathrm{P} * \mathrm{R} \sqsubseteq^{\mathcal{E}} \mathrm{Q} * \mathrm{R}
$$

AFrame

$\Gamma\left|\Phi \vdash(\Delta) .\langle\mathrm{P}\rangle c\langle\mathrm{Q}\rangle^{\mathcal{E}} \quad \Gamma, \Delta\right| \Phi \vdash$ stable $(\mathrm{R})$ $\Gamma \mid \Phi \vdash(\Delta) .\langle\mathrm{P} * \Delta \mathrm{R}\rangle c\langle\mathrm{Q} * \mathrm{R}\rangle^{\mathcal{E}}$

$$
\frac{\stackrel{\text { Frame }}{\Gamma \mid \Phi \vdash(\Delta) .\{\mathrm{P}\}} c\{\mathrm{Q}\} \quad \Gamma, \Delta \mid \Phi \vdash \operatorname{stable}(\mathrm{R})}{\Gamma \mid \Phi \vdash(\Delta) .\{\mathrm{P} * \mathrm{R}\} c\{\mathrm{Q} * \mathrm{R}\}}
$$

\section{Conseq}

$\Gamma, \Delta\left|\Phi \vdash \mathrm{P}_{1} \sqsubseteq^{\mathcal{E}} \mathrm{P}_{2} \quad \Gamma\right| \Phi \vdash(\Delta) .\left\{\mathrm{P}_{2}\right\} c\left\{\mathrm{Q}_{2}\right\} \quad \Gamma, \Delta \mid \Phi \vdash \mathrm{Q}_{2} \sqsubseteq^{\mathcal{E}} \mathrm{Q}_{1}$
$\Gamma \mid \Phi \vdash(\Delta) .\left\{\mathrm{P}_{1}\right\} c\left\{\mathrm{Q}_{1}\right\}$

ACONSEQ

$\Gamma, \Delta\left|\Phi \vdash \mathrm{P}_{1} \sqsubseteq^{\mathcal{E}} \mathrm{P}_{2} \quad \Gamma\right| \Phi \vdash(\Delta) .\left\langle\mathrm{P}_{2}\right\rangle c\left\langle\mathrm{Q}_{2}\right\rangle^{\mathcal{E}} \quad \Gamma, \Delta \mid \Phi \vdash \mathrm{Q}_{2} \sqsubseteq^{\mathcal{E}} \mathrm{Q}_{1}$
$\Gamma \mid \Phi \vdash(\Delta) .\left\langle\mathrm{P}_{1}\right\rangle c\left\langle\mathrm{Q}_{1}\right\rangle^{\mathcal{E}}$

$$
\begin{aligned}
& \text { SEQ } \\
& \frac{\Gamma\left|\Phi \vdash(\Delta) .\{P\} s_{1}\{Q\} \quad \Gamma\right| \Phi \vdash(\Delta) .\{Q\} s_{2}\{R\}}{\Gamma \mid \Phi \vdash(\Delta) .\{P\} s_{1} ; s_{2}\{R\}}
\end{aligned}
$$

